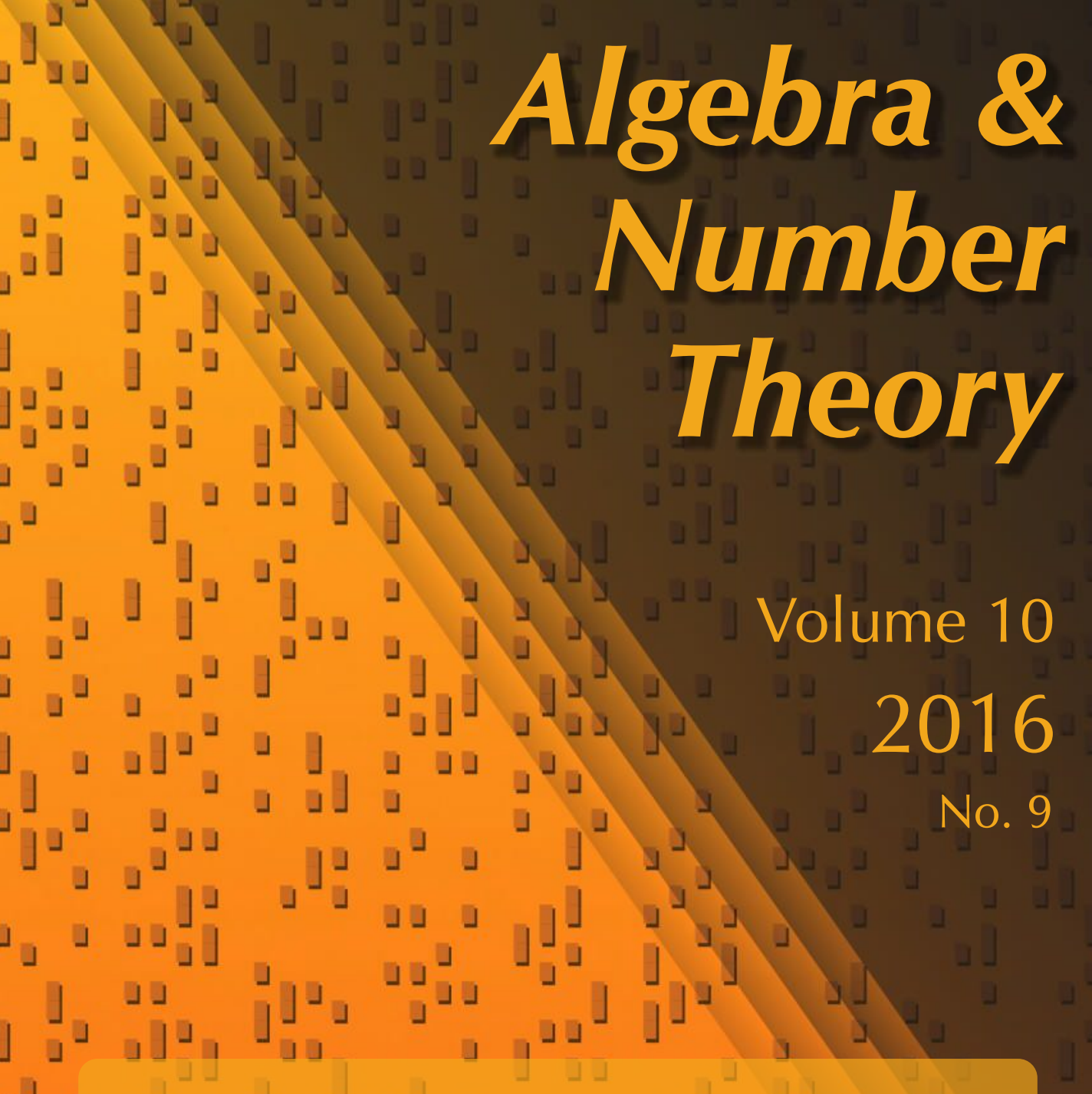




\title{
Galois-generic points on Shimura varieties
}

\author{
Anna Cadoret and Arno Kret
}

\begin{abstract}
We discuss existence and abundance of Galois-generic points for adelic representations attached to Shimura varieties. First, we show that, for Shimura varieties of abelian type, $\ell$-Galois-generic points are Galois-generic; in particular, adelic representations attached to such Shimura varieties admit ("lots of") closed Galoisgeneric points. Next, we investigate further the distribution of Galois-generic points and show the André-Pink conjecture for them: if $S$ is a connected Shimura variety associated to a $\mathbb{Q}$-simple reductive group, then every infinite subset of the generalized Hecke orbit of a Galois-generic point is Zariski-dense in S. Our proof follows the approach of Pink for Siegel Shimura varieties. Our main contribution consists in showing that there are only finitely many Hecke operators of bounded degree on (adelic and connected) Shimura varieties. Compared with other approaches of this result, our proof, which relies on Bruhat-Tits theory, is effective and works for arbitrary Shimura varieties.
\end{abstract}

\section{Introduction}

Given a smooth, separated and geometrically connected scheme $S$ over a field $k$ and a point $s \in S$, let $\sigma_{s}: \pi_{1}(s) \rightarrow \pi_{1}(S)$ denote the morphism induced by functoriality of the étale fundamental group. ${ }^{1}$ Given an algebraic group $G$ over $\mathbb{Q}$ and an adelic representation $\rho: \pi_{1}(S) \rightarrow G\left(\mathbb{A}_{f}\right)$, let $\rho_{\ell}: \pi_{1}(S) \rightarrow G\left(\mathbb{A}_{f}\right) \rightarrow G\left(\mathbb{Q}_{\ell}\right)$ denote its $\ell$-adic component. We say that $s \in S$ is Galois-generic with respect to $\rho: \pi_{1}(S) \rightarrow G\left(\mathbb{A}_{f}\right)$ if the image of $\rho \circ \sigma_{s}$ is open in the image of $\rho$, and $\ell$-Galois-generic if the image of $\rho_{\ell} \circ \sigma_{s}$ is open in the image of $\rho_{\ell}$.

To a Shimura datum $(G, X)$ and a neat compact open subgroup $K_{0} \subset G\left(\mathbb{A}_{f}\right)$, we can attach a representation $\rho_{K_{0}}: \pi_{1}\left(S\left[K_{0}\right]\right) \rightarrow K_{0} \subset G\left(\mathbb{A}_{f}\right)$ of the étale fundamental group, where $S\left[K_{0}\right] \subset \operatorname{Sh}_{K_{0}}(G, X)$ is a geometrically connected component (defined over a finite extension $E\left[K_{0}\right]$ of the reflex field $E=E(G, X)$ of $(G, X))$. This representation group-theoretically encodes the tower of étale covers $\operatorname{Sh}_{K}(G, X) \rightarrow \operatorname{Sh}_{K_{0}}(G, X)$ indexed by open subgroups $K \subset K_{0}$. For a

MSC2010: primary 11G18; secondary 20G35, 14F20.

Keywords: Shimura varieties, Hecke orbits, Adelic representations of étale fundamental group,

Galois generic points.

${ }^{1}$ Recall that $\pi_{1}(s)$ identifies with the absolute Galois group of the residue field $k(s)$ at $s$. 
point $s\left[K_{0}\right] \in S\left[K_{0}\right]$ and a field extension $F$ of $E\left[K_{0}\right]$, we say that the induced point $s\left[K_{0}\right]_{F} \in S\left[K_{0}\right]_{F}$ is Galois-generic if it is Galois-generic with respect to $\left.\rho_{K_{0}}\right|_{\pi_{1}\left(S\left[K_{0}\right]_{F}\right)}: \pi_{1}\left(S\left[K_{0}\right]_{F}\right) \rightarrow G\left(\mathbb{A}_{f}\right)$, and $\ell$-Galois-generic if it is $\ell$-Galois-generic with respect to $\left.\rho\left[K_{0}\right]_{\ell}\right|_{\pi_{1}\left(S\left[K_{0}\right]_{F}\right)}: \pi_{1}\left(S\left[K_{0}\right]_{F}\right) \rightarrow G\left(\mathbb{Q}_{\ell}\right)$. We say that a point $s$ in $\mathrm{Sh}(G, X)_{F}$ is $(\ell-)$ Galois-generic if for some (equivalently, any) neat compact open subgroup $K_{0} \subset G\left(\mathbb{A}_{f}\right)$, the image $s\left[K_{0}\right]$ of $s$ in $\operatorname{Sh}_{K_{0}}(G, X)_{F}$ is $(\ell-)$ Galois-generic.

In the context of Shimura varieties, the terminology "Galois-generic" was introduced by Pink [2005, Definition 6.3]. The definition of Pink does not resort to the formalism of étale fundamental groups and is seemingly stronger than ours. Namely, if $E^{\mathrm{ab}}$ denotes the maximal abelian extension of $E$, a point $s\left[K_{0}\right] \in S\left[K_{0}\right]$ is Galoisgeneric in the sense of Pink if and only if the induced point $s\left[K_{0}\right]_{E^{\mathrm{ab}}} \in S\left[K_{0}\right]_{E^{\mathrm{ab}}}$ is Galois-generic in our sense. However, using the facts that $\rho\left(\pi_{1}\left(S\left[K_{0}\right]_{\bar{E}}\right)\right)=\Gamma_{0}^{-}$, where $\Gamma_{0}^{-} \subset G\left(\mathbb{A}_{f}\right)$ denotes the adelic closure of $\Gamma_{0}:=G(\mathbb{Q}) \cap K$ in $G\left(\mathbb{A}_{f}\right)$, and that every open subgroup of $\Gamma_{0}^{-}$has finite abelianization (Theorem 5.4), we show in Proposition 6.1.1 that the two definitions coïncide.

1.1. Existence. Given a scheme $S$ smooth, separated and geometrically connected over a field $k$, and an adelic representation $\rho: \pi_{1}(S) \rightarrow G\left(\mathbb{A}_{f}\right)$, the first question which arises is whether there exists Galois-generic points (other than the generic point) with respect to $\rho$. While $\ell$-adic specialization techniques give rise to "lots of" closed $\ell$-Galois-generic points (see 3.3.1), they fail to ensure the existence of a single closed point which is $\ell$-Galois-generic for every prime $\ell$ (hence a fortiori which is Galois-generic).

However, for adelic representations attached to motives, the $\ell$-adic Tate conjectures say that a point that is $\ell$-Galois-generic for one prime $\ell$ is $\ell$-Galois-generic for every prime $\ell$, and the modulo- $\ell$ variant of the Tate conjectures even predict that a point which is $\ell$-Galois-generic for one prime $\ell$ is Galois-generic (see 3.3.2).

By works of Faltings (e.g., [Faltings and Wüstholz 1984]), partial forms of the modulo- $\ell$ variant of the Tate conjectures are available for abelian varieties; this is enough to ensure that for adelic representations attached to the Tate module of abelian schemes, a point which is $\ell$-Galois-generic for one prime $\ell$ is Galois-generic [Cadoret 2015, Theorem 1.2] (see Theorem 3.3.2.2). The first main result of this paper is the extension of this statement to adelic representations attached to Shimura varieties of abelian type (see 6.3.2 for the definition of "abelian type").

Theorem A. Assume $(G, X)$ is a Shimura datum of abelian type. Then a point $s \in \operatorname{Sh}(G, X)$ is $\ell$-Galois-generic if and only if it is Galois-generic.

The bridge between [Cadoret 2015, Theorem 1.2] and adelic representations attached to Shimura varieties is provided by the moduli description of Siegel Shimura varieties. The remaining parts of the argument rely on the general machinery of Shimura varieties and group-theoretical arguments. Our approach fails to handle 
the case of Shimura data $(G, X)$ which are not of abelian type, though it seems reasonable to expect that Theorem A should also hold for such representations.

Theorem A and the description of Galois-generic points in terms of adelic representations are also used in [Cadoret and Moonen 2015] to prove that, for motives parametrized by Shimura varieties of abelian type (e.g., abelian varieties, $K 3$ surfaces) the integral and adelic variants of the Mumford-Tate conjecture follow from the standard ( $\ell$-adic) Mumford-Tate conjecture.

\subsection{Equidistribution and the André-Pink conjecture. Theorem A implies that} adelic representations attached to Shimura varieties of abelian type admit "lots of" Galois-generic points since they admit "lots of" $\ell$-Galois-generic points. For instance, combining Theorem $A$ and the abundance result for $\ell$-Galois-generic points of [Cadoret and Tamagawa 2013] (see Fact 3.3.1.2), on can show the following. Say that an irreducible curve $C \hookrightarrow S\left[K_{0}\right]$ is Galois-generic if its generic point is. Then the set of irreducible Galois-generic curves defined over a number field is Zariski-dense in $S\left[K_{0}\right]$, and for each such curve $C \hookrightarrow S\left[K_{0}\right]$, defined over a finite extension $E_{C}$ of $E\left[K_{0}\right]$ and integer $d \geq 1$, all but finitely many closed points $t \in C$ with $\left[k(t): E_{C}\right] \leq d$ are Galois-generic. In particular, closed Galois-generic points are Zariski-dense, which is not surprising once their existence is proved: being Galois-generic is preserved by Hecke operators and Hecke orbits are Zariski dense. But the restrictions on the degree show more. Indeed, it follows from the definition of Galois-generic points and the fact (see Theorem 7.2.2) that there are only finitely many Hecke operators of bounded degree on a connected Shimura variety, that for every Galois-generic point $t \in S\left[K_{0}\right]$ and integer $d \geq 1$ there are only finitely many $t^{\prime}$ in the Hecke orbit of $t$ with $\left[k\left(t^{\prime}\right): E\right] \leq d$. Thus there are infinitely many Hecke orbits of closed Galois-generic points on Shimura varieties of abelian type, and even infinitely many Hecke orbits of closed Galois-generic points intersecting a Galois-generic curve defined over a number field.

Using equidistribution techniques, we can strengthen these results as follows. Let $(G, X)$ be a Shimura datum (which we no longer assume to be of abelian type). Let $K_{0} \subset G\left(\mathbb{A}_{f}\right)$ be a neat compact open subgroup and let $X^{+} \subset X$ be a connected component. Write $\Gamma_{0}:=K_{0} \cap G(\mathbb{Q})_{+}$, where $G(\mathbb{Q})_{+} \subset G(\mathbb{Q})$ denotes the stabilizer of $X^{+}$. Eventually, let $S\left[K_{0}\right]=: \operatorname{Sh}_{\Gamma_{0}}\left(G, X^{+}\right) \subset \operatorname{Sh}_{K_{0}}(G, X)$ denote the geometrically connected component containing the image of $X^{+} \times\{1\}$ (that is, $\left.\operatorname{Sh}_{\Gamma_{0}}\left(G, X^{+}\right)_{\mathbb{C}} \simeq \Gamma_{0} \backslash X^{+}\right)$. Write $\operatorname{Aut}\left(G, X^{+}\right)$for the group of automorphisms of $G$ defined over $\mathbb{Q}$ and stabilizing $X^{+}$. For every $S\left[K_{0}\right] \in \operatorname{Sh}_{\Gamma_{0}}\left(G, X^{+}\right)$, write

$$
\widehat{T}_{\Gamma_{0}}\left(s\left[K_{0}\right]\right):=\bigcup_{\phi \in \operatorname{Aut}\left(G, X^{+}\right)} T_{\Gamma_{0}, \phi}\left(s\left[K_{0}\right]\right)
$$

for the (full) generalized Hecke orbit of $s\left[K_{0}\right]$, where $T_{\Gamma_{0}, \phi}$ denotes the generalized Hecke operator induced by $\phi$ on $\operatorname{Sh}_{\Gamma_{0}}\left(G, X^{+}\right)$(see Section 7.1). 
Theorem B (André-Pink conjecture for Galois-generic points [André 1989, Chapter X, Problem 3; Pink 2005, Conjecture 1.6; Orr 2013, Conjecture 1.3]). Assume $G$ is almost $\mathbb{Q}$-simple. Then for every Galois-generic point $s\left[K_{0}\right] \in \operatorname{Sh}_{\Gamma_{0}}\left(G, X^{+}\right)$, every infinite subset of $\widehat{T}_{\Gamma_{0}}\left(s\left[K_{0}\right]\right)$ is Zariski-dense in $\mathrm{Sh}_{\Gamma_{0}}\left(G, X^{+}\right)$.

For Shimura varieties of abelian type a consequence of Theorem A, Theorem B and [Cadoret and Tamagawa 2013] is that if $C \hookrightarrow S$ is an irreducible Galois-generic curve defined over a number field, then $C$ is cut by infinitely many Hecke orbits of closed Galois-generic points, and each of these Hecke orbits cuts $C$ in only finitely many points.

Theorem B extends a previous result of Pink [2005, Theorem 7.6] for the Siegel Shimura varieties; our proof follows that of Pink but with some technical adjustments required to deal with non-simply connected groups $G$. More precisely, the main ingredient in Pink's argument is an equidistribution result of Clozel, Oh and Ullmo [Clozel et al. 2001, Theorem 1.6, Remark (3)] for $\mathrm{GSp}_{2 g}$. To deal with arbitrary Shimura varieties, one needs a generalization of [Clozel et al. 2001, Theorem 1.6] for adjoint groups $G$ and arithmetic (not only congruence) subgroups $\Gamma \subset G(\mathbb{Q})$. Such a generalization was proved by Eskin and Oh [2006, Theorem 1.2] following an idea of Burger and Sarnak [1991] (see Section 7.2). To apply Eskin and Oh's result to our situation, we have to ensure that, for an arithmetic subgroup $\Gamma \subset G(\mathbb{Q})$, there are only finitely many Hecke operators $T_{a}, a \in G(\mathbb{Q})$ with $|\Gamma \backslash \Gamma a \Gamma|$ bounded (see Theorem 7.2.2). We provide a proof of this result in Section 8, proceeding in two steps: First, we prove Theorem 8.2.1, the adelic variant of Theorem 7.2.2; here the "natural" tools are avatars of the Bruhat-Tits decomposition, which give explicit estimates for the local degrees (see Section 8.2 for details). Then, we deduce Theorem 7.2.2 from this adelic variant by reducing to the simply connected case, where we can apply strong approximation.

After a first version of this paper was released, we were informed by Hee Oh that Theorem 7.2.2 could also be proved by equidistribution arguments like those used in [Eskin and Oh 2006], but this proof does not seem to be effective, nor does it work for adelic Hecke operators (see Section 9.1 for details).

If we restrict to connected Shimura varieties of abelian type, Theorem B can easily be recovered from Orr's thesis [2013, Theorem 1.5(ii)] arguing as follows. Let $s\left[K_{0}\right] \in \mathrm{Sh}_{\Gamma_{0}}\left(G, X^{+}\right)$be a Galois-generic point, let $A$ be an infinite subset of $\widehat{T}_{\Gamma_{0}}\left(s\left[K_{0}\right]\right)$, and let $Z$ be some irreducible component of the Zariski-closure of $A$ containing $s\left[K_{0}\right]$. Since $s\left[K_{0}\right]$ is Galois-generic, it is Hodge-generic (see Proposition 6.2.1). Hence, the smallest special subvariety of $\operatorname{Sh}_{\Gamma_{0}}\left(G, X^{+}\right)$containing $s\left[K_{0}\right]$ is equal to the smallest special subvariety of $\operatorname{Sh}_{\Gamma_{0}}\left(G, X^{+}\right)$containing $Z$ (because in both cases, this subvariety has to be $\operatorname{Sh}_{\Gamma_{0}}\left(G, X^{+}\right)$itself). By construction, $A \cap Z$ is Zariski-dense in $Z$; hence, from [Orr 2013, Theorem 1.5(ii)], $Z$ is weakly special. But as $Z$ contains the Hodge-generic point $s\left[K_{0}\right]$, this forces 
$Z=\operatorname{Sh}_{\Gamma_{0}}\left(G, X^{+}\right)$. Orr's approach to Theorem B relies on different techniques than ours (Masser-Wüstholz isogeny bound for abelian varieties and o-minimality); it is more general since it works for Hodge-generic points but, due to the use of the Masser-Wüstholz isogeny bound, it only applies to Shimura varieties of abelian type. For connected Shimura varieties of abelian type, one can also give a proof of Theorem 7.2.2 based on the Masser-Wüstholz isogeny bound and the existence of closed Galois-generic points (see Section 9.2 for details).

In Section 3, we review the notion of Galois-generic points attached to adelic representations of the étale fundamental group and some of their basic properties. We also recall the main existence and abundance results for $\ell$-Galois-generic points and discuss in more details the relation between $\ell$-Galois generic points and Galois-generic points for motivic representations. In Section 4, we construct the adelic representations attached to Shimura varieties and review some of their basic properties. Section 5 is technical and gathers the group-theoretical results about the adelic closure of arithmetic subgroups of semisimple groups that we need to prove Theorem A. In Section 6, we focus on Galois-generic points attached to Shimura varieties, show that our definition coïncides with the one of Pink, that Galois-generic-points are Hodge generic, and complete the proof of Theorem A. Section 7 is devoted to the proof of Theorem B. The proof of Theorem 7.2.2 is postponed to Section 8 as it can be read independently of the rest of the paper and involves techniques of a different nature. In the final Section 9, we discuss alternative approaches to Theorem 7.2.2: a noneffective approach based on equidistribution (see Section 9.1) and an effective approach (limited to connected Shimura varieties of abelian type) based on the Masser-Wüstholz isogeny theorem (see Section 9.2).

\section{Notation and conventions}

The fields in this paper, when of characteristic 0 , will always be assumed to be embedded into the field $\mathbb{C}$ of complex numbers. For such fields, compositum, Galois, abelian, algebraic closures, etc., will always mean with respect to the given embedding into $\mathbb{C}$.

Given schemes $S$ and $T$ over a field $k$, unless there is a risk of confusion, we write $S_{T}:=S \times_{k} T$ (that is, we omit the notation for the base field $k$ ). When $T=\operatorname{spec}(F)$ for a field extension $k \subset F$, we write $S_{F}:=S_{\operatorname{spec}(F)}$. However, when $S=: s=\operatorname{spec}(E)$ for a field extension $k \subset E$, and $k \subset F$ is another field extension with $E, F$ embedded into $\mathbb{C}$, we write $s_{F}:=\operatorname{spec}(E F)$; that is, we implicitly pick the connected component of $s \times \times_{\operatorname{spec}(k)} \operatorname{spec}(F)$ corresponding to the given embeddings of $E, F$ in $\mathbb{C}$.

Given a scheme $S$ of finite type over a field $k$ and a point $s \in S$, we write $k(s)$ for the residue field (a finitely generated extension of $k$ ) of $S$ at $s$. We identify points $s \in S$ and the corresponding morphisms of $k$-schemes $s: \operatorname{spec}(k(s)) \rightarrow S$. For a point 
$s \in S$, a geometric point above $s$ is a morphism $\bar{s}: \operatorname{spec}(\Omega) \rightarrow S$ factorizing through $s: \operatorname{spec}(k(s)) \rightarrow S$ and such that $\Omega$ is an algebraically closed field. In general, we do not specify the algebraically closed field $\Omega$ in the notation for geometric points (see below) and for a point $s \in S$, unless otherwise specified $\bar{s}$ will always denote a geometric point over $s$. For every $s \in S$ and geometric point $\bar{s}$ over $s$, let $F_{\bar{s}}$ denote the associated fiber functor from étale covers of $S$ to sets. Recall that, by definition, the étale fundamental group of $S$ based at $\bar{s}$ is the automorphism group $\pi_{1}(S, \bar{s})$ of $F_{\bar{s}}$ and that, if $S$ is connected, for every $s, s^{\prime} \in S$ there always exists isomorphisms of fiber functors $\alpha: F_{\bar{S}} \stackrel{\sim}{\rightarrow} F_{\bar{s}^{\prime}}$, and the set of such isomorphisms is a $\pi_{1}(S, \bar{s})$-torsor. In particular, for every étale cover $X \rightarrow S, \alpha$ yields a bijection $\alpha: F_{\bar{S}}(X) \stackrel{\sim}{\rightarrow} F_{\bar{s}^{\prime}}(X)$ which is equivariant with respect to the isomorphism of étale fundamental groups

$$
\pi_{1}(S, \bar{s}) \stackrel{\sim}{\rightarrow} \pi_{1}\left(S, \bar{s}^{\prime}\right), \quad \sigma \mapsto \alpha \sigma \alpha^{-1} .
$$

Thus, unless it helps to understand the situation, we will omit the base-point $\bar{s}$ in our notation for étale fundamental groups. Given a field $k$, we often shorten $\pi_{1}(\operatorname{spec}(k))$ in $\pi_{1}(k)$, which identifies with the absolute Galois group of $k$. For a point $s \in S$, we also write $\pi_{1}(s)=\pi_{1}(k(s))$.

For an algebraic group $G$, we let $G^{\text {der }} \subset G$ denote its derived subgroup, $Z(G) \subset G$ its center, $p^{\mathrm{ab}}: G \rightarrow G^{\mathrm{ab}}:=G / G^{\mathrm{der}}$ its abelianization and $p^{\mathrm{ad}}: G \rightarrow G^{\mathrm{ad}}:=G / Z(G)$ its adjoint quotient. If $G$ is semisimple, we write $p^{\text {sc }}: G^{\text {sc }} \rightarrow G$ for its simply connected cover and set $\mu_{G}:=\operatorname{ker}\left(p^{\mathrm{sc}}\right)$. Let $\mathbb{A}_{f}$ denote the ring of finite adeles of $\mathbb{Q}$. Given a subgroup $\Gamma \subset G\left(\mathbb{A}_{f}\right) \subset \prod_{\ell} G\left(\mathbb{Q}_{\ell}\right)$, we write $\Gamma_{\ell} \subset G\left(\mathbb{Q}_{\ell}\right)$ for the projection of $\Gamma$ into $G\left(\mathbb{Q}_{\ell}\right)$.

\section{Galois-generic and strictly Galois-generic points}

Let $k$ be a field of characteristic 0 and let $S$ be a smooth, separated and geometrically connected scheme over $k$ with generic point $\eta$.

3.1. Galois-generic points. Let $\Gamma$ be a topological group and $\rho: \pi_{1}(S) \rightarrow \Gamma$ a continuous group morphism. Write

$$
\Pi_{\rho}:=\operatorname{im}(\rho), \quad \bar{\Pi}_{\rho}:=\rho\left(\pi_{1}\left(S_{\bar{k}}\right)\right) .
$$

Every point $s \in S$ induces by functoriality of the étale fundamental group a morphism of profinite groups $\sigma_{s}: \pi_{1}(s) \rightarrow \pi_{1}(S)$ which is a section of the canonical projection $\pi_{1}\left(S_{k(s)}\right) \rightarrow \pi_{1}(k(s))$. Write

$$
\Pi_{\rho, s}:=\operatorname{im}\left(\rho \circ \sigma_{s}\right) \subset \Pi_{\rho}, \quad \bar{\Pi}_{\rho, s}:=\bar{\Pi}_{\rho} \cap \Pi_{\rho, s} .
$$

Definition 3.1.1. We say that $s \in S$ is Galois-generic with respect to $\rho$ if $\Pi_{\rho, s}$ is open in $\Pi_{\rho}$, and strictly Galois-generic with respect to $\rho$ if $\Pi_{\rho, s}=\Pi_{\rho}$. 
We use this terminology when $\Gamma=G\left(\mathbb{A}_{f}\right)$ for some algebraic group $G$ over $\mathbb{Q}$. For every prime $\ell$, write

$$
\rho_{\ell}: \pi_{1}(S) \stackrel{\rho}{\rightarrow} G\left(\mathbb{A}_{f}\right) \subset \prod_{\ell} G\left(\mathbb{Q}_{\ell}\right) \rightarrow G\left(\mathbb{Q}_{\ell}\right)
$$

for the $\ell$-adic component of $\rho: \pi_{1}(S) \rightarrow G\left(\mathbb{A}_{f}\right)$. If $\rho: \pi_{1}(S) \rightarrow G\left(\mathbb{A}_{f}\right)$ is clear from the context, we omit the subscript $(-)_{\rho}$ in the notation $\Pi_{\rho}, \bar{\Pi}_{\rho}, \Pi_{\rho, s}$, etc., and simply say that $s \in S$ is Galois-generic (resp. $\ell$-Galois-generic) if $s \in S$ is Galois-generic with respect to $\rho$ (resp. $\rho_{\ell}$ ). Similarly, we say that $s \in S$ is strictly Galois-generic (resp. strictly $\ell$-Galois-generic) if $s \in S$ is strictly Galois-generic with respect to $\rho$ (resp. $\rho_{\ell}$ ).

\subsection{Elementary properties of Galois-generic and strictly Galois-generic points.}

3.2.1. As $S$ is normal, $\eta \in S$ is strictly Galois-generic.

3.2.2. Let $k \subset \tilde{k}$ be a finitely generated field extension. Then $s \in S$ is Galois-generic with respect to $\rho$ if and only if $s_{\tilde{k}} \in S_{\tilde{k}}$ is Galois-generic with respect to $\left.\rho\right|_{\pi_{1}\left(S_{\tilde{k}}\right)}$. This follows from the fact that the images of the canonical morphisms $\pi_{1}\left(s_{\tilde{k}}\right) \rightarrow \pi_{1}(s)$ and $\pi_{1}\left(S_{\tilde{k}}\right) \rightarrow \pi_{1}(S)$ are open.

3.2.3. As $S$ is geometrically connected over $k$, we have a short exact sequence

$$
1 \rightarrow \pi_{1}\left(S_{\bar{k}}\right) \rightarrow \pi_{1}(S) \rightarrow \pi_{1}(k) \rightarrow 1
$$

and as the image of $\pi_{1}(s) \stackrel{\sigma_{s}}{\rightarrow} \pi_{1}(S) \rightarrow \pi_{1}(k)$ is open in $\pi_{1}(k)$, we see that $s \in S$ is Galois-generic with respect to $\rho$ if and only if $\bar{\Pi}_{s}$ is open in $\bar{\Pi}$. Another way to formulate this observation is the following. Let $k \subset \tilde{k} \subset \bar{k}$ denote the (in general infinite) Galois subextension corresponding to the image of $\rho^{-1}(\bar{\Pi}) \subset \pi_{1}(S)$ by $\pi_{1}(S) \rightarrow \pi_{1}(k)$. Then $s \in S$ is Galois-generic with respect to $\rho$ if and only if $s_{\tilde{k}} \in S_{\tilde{k}}$ is Galois-generic with respect to $\left.\rho\right|_{\pi_{1}\left(S_{\tilde{k}}\right)}$. Under additional assumptions, one can enlarge $\tilde{k}$. For instance:

Lemma 3.2.4. Assume that every open subgroup of $\bar{\Pi}$ has finite abelianization. With the above notation, let $\tilde{k} \subset \tilde{k}^{\mathrm{ab}} \subset \bar{k}$ denote the maximal abelian extension of $\tilde{k}$ in $\bar{k}$. Then $s \in S$ is Galois-generic with respect to $\rho$ if and only if $s_{\tilde{k}^{\mathrm{ab}}} \in S_{\tilde{k}^{\mathrm{ab}}}$ is Galois-generic with respect to $\left.\rho\right|_{\pi_{1}\left(S_{\tilde{k}_{\mathrm{ab}}}\right)}$.

Proof. The nontrivial implication is the "only if" one. So, assume that $s \in S$ is Galois-generic with respect to $\rho$. Then $\Pi_{s_{\tilde{k}}} \subset \bar{\Pi}$ is open. In particular, it has finite abelianization. Thus its quotient $\Pi_{s_{\tilde{k}}} \rightarrow \Pi_{s_{\tilde{k}}} / \Pi_{s_{\tilde{k}} \text { ab }}$ (which, being a quotient of $\operatorname{Gal}\left(\tilde{k}^{\mathrm{ab}} \mid \tilde{k}\right)$, is abelian) is finite.

3.2.5. If $S^{\prime} \rightarrow S$ is a dominant morphism of finite type with $S^{\prime}$ connected (for instance, a connected étale cover) and $s^{\prime} \in S^{\prime}$ a point above $s \in S$, then $s \in S$ is Galois-generic with respect to $\rho$ if and only if $s^{\prime} \in S^{\prime}$ is Galois-generic with respect to $\left.\rho\right|_{\pi_{1}\left(S^{\prime}\right)}$. 
3.2.6. Given a Galois-generic point $s \in S$, one can always find a connected étale cover $S^{\prime} \rightarrow S$ and $s^{\prime} \in S^{\prime}$ above $s$ such that $s^{\prime} \in S^{\prime}$ is strictly Galois-generic with respect to $\left.\rho\right|_{\pi_{1}\left(S^{\prime}\right)}$. Indeed, let $s \in S$ be a Galois-generic point and let $U \subset \Pi$ be any open subgroup contained in $\Pi_{s}$. Let $S_{U} \rightarrow S$ denote the connected étale cover corresponding to the open subgroup $\rho^{-1}(U) \subset \pi_{1}(S)$ and let $k(s) \hookrightarrow k(s)_{U}$ denote the finite field extension corresponding to the open subgroup $\left(\rho \circ \sigma_{s}\right)^{-1}(U) \subset \pi_{1}(s)$. Then $s \in S$ lifts to a $k(s)_{U}$-rational point $s_{U} \in S_{U}$ which is strictly Galois-generic with respect to $\left.\rho\right|_{\pi_{1}\left(S_{U}\right)}$.

By definition, if $s \in S$ is strictly Galois-generic, then for every open subgroup $U \subset \Pi$ and corresponding connected étale cover $S_{U} \rightarrow S, \pi_{1}(s)$ acts transitively on the geometric fiber of $S_{U} \rightarrow S$ over $s$.

3.3. The $\mathbf{l}-\boldsymbol{G} \boldsymbol{G} \Leftrightarrow \boldsymbol{G} \boldsymbol{G}$ problem. We now assume that $\Gamma=G\left(\mathbb{A}_{f}\right)$. Let $S^{\mathrm{gg}} \subset S$ and $S_{\ell}^{\mathrm{gg}} \subset S$ denote the sets of Galois- and $\ell$-Galois-generic points, respectively. Write

$$
S_{\infty}^{\mathrm{gg}}:=\bigcap_{\ell} S_{\ell}^{\mathrm{gg}}, \quad S^{\mathrm{gg} \infty}:=\bigcup_{\ell} S_{\ell}^{\mathrm{gg}} .
$$

Clearly,

$$
S^{\mathrm{gg}} \subset S_{\infty}^{\mathrm{gg}} \subset S^{\mathrm{gg} \infty}
$$

3.3.1. $\ell$-Galois-generic points. One can show by $\ell$-adic specialization techniques that $S_{\ell}^{\mathrm{gg}}$ is nonempty and even "large in an arithmetical sense" provided $k$ satisfies some reasonable assumptions. More precisely, we have the following facts.

Fact 3.3.1.1 [Serre 1989, §10.6]. Assume $k$ is Hilbertian. Then there exist an integer $d \geq 1$ and infinitely many closed strictly $\ell$-Galois-generic points $s \in S$ with $[k(s): k] \leq d$.

Fact 3.3.1.2 [Cadoret and Tamagawa 2013, Theorem 1.1]. Assume $k$ is finitely generated, $S$ is a curve and every open subgroup of $\bar{\Pi}_{\ell}$ has finite abelianization. Then for every integer $d \geq 1$, all but finitely many $s \in S$ with $[k(s): k] \leq d$ are $\ell$-Galois-generic.

Note that $\ell$-adic motivic representations (see Section 3.3.2) satisfy the assumption of Fact 3.3.1.2 [Cadoret and Tamagawa 2012, §5.2]. The $\ell$-adic components of adelic representations attached to Shimura varieties also satisfy this assumption (see Section 4.2 and Theorem 5.4).

These results rely heavily on the fact that $\bar{\Pi}_{\ell}$ and $\Pi_{\ell}$ are compact $\ell$-adic Lie groups: the key point in the proof of Fact 3.3.1.1 is that the Frattini subgroup $\Phi(\Pi)$ of a compact $\ell$-adic Lie group $\Pi$ is open in $\Pi$. This property is also crucial in the proof of Fact 3.3.1.2, which also involves finer structural results about compact $\ell$-adic Lie groups. 
3.3.2. Motivic representations. The above results are $\ell$-adic in nature and fail to ensure that $S_{\infty}^{\mathrm{gg}}$ contains points other than the generic point.

Definition 3.3.2.1. We say that an adelic representation $\rho: \pi_{1}(S) \rightarrow G\left(\mathbb{A}_{f}\right)$ satisfies the $(\ell-\mathrm{GG} \Leftrightarrow \mathrm{GG})$-property if $S^{\mathrm{gg}}=S_{\infty}^{\mathrm{gg}}=S^{\mathrm{gg} \infty}$.

When an adelic representation satisfies the ( $\ell$-GG $\Leftrightarrow \mathrm{GG})$-property, the abundance results of Section 3.3.1 automatically hold for Galois-generic points.

Conjecturally, motivic representations - those of the form

$$
\rho_{X}^{w}: \pi_{1}(S) \rightarrow \prod_{\ell} \mathrm{GL}\left(\mathrm{H}^{w}\left(X_{\bar{\eta}}, \mathbb{Q}_{\ell}\right)\right)
$$

for some smooth projective scheme $f: X \rightarrow S$ - should satisfy the ( $\ell$-GG $\Leftrightarrow$ GG)property. More precisely, the equality $S_{\infty}^{\mathrm{gg}}=S^{\mathrm{gg} \infty}$ follows from the Tate conjectures [Serre 1994, §9; André 2004, §7.3] while the equality $S^{\mathrm{gg}}=S_{\infty}^{\mathrm{gg}}$ follows from the modulo- $\ell$ variant of the Tate conjectures proposed by Serre $[1994, \S 10]$.

For abelian schemes, partial forms of the modulo- $\ell$ variants of the Tate conjectures were proved by Faltings; see, e.g., [Faltings and Wüstholz 1984]. These are enough to show that adelic motivic representations attached to abelian schemes satisfy the ( $\ell$-GG $\Leftrightarrow \mathrm{GG}$ )-property. More precisely, given an abelian scheme $f: X \rightarrow S$, recall that $R^{w} f_{*} \mathbb{Z}_{\ell}=\Lambda^{w} R^{1} f_{*} \mathbb{Z}_{\ell} \simeq \Lambda^{w} T_{\ell}(X)^{\vee}$, where

$$
T_{\ell}(X):=\lim _{\overleftarrow{n}} X\left[\ell^{n}\right]
$$

denotes the $\ell$-adic Tate module (here $X[N]$ denotes the kernel of multiplication by $N$ on $X$; as $k$ has characteristic 0 , this is an étale cover of $S$ ). Thus, the $(\ell$-GG $\Leftrightarrow \mathrm{GG})$-property for $\rho_{X}^{w}: \pi_{1}(S) \rightarrow \prod_{\ell} \mathrm{GL}\left(\mathrm{H}^{w}\left(X_{\bar{\eta}}, \mathbb{Q}_{\ell}\right)\right)$ boils down to the following statement.

Theorem 3.3.2.2 [Cadoret 2015, Theorem 1.2]. The representation

$$
\left(\rho_{X}^{1}\right)^{\vee}: \pi_{1}(S) \rightarrow \operatorname{GL}\left(T(X)_{\bar{\eta}}\right)
$$

satisfies the $(\ell-G G \Leftrightarrow G G)$-property.

\section{Adelic representations attached to Shimura varieties}

Let $(G, X)$ be a Shimura datum. We assume throughout that $G$ is the generic Mumford-Tate group on $X$. In this case, conditions 2.1.1.1-2.1.1.5 of [Deligne 1979] are satisfied and $Z(\mathbb{Q})$ is discrete in $Z\left(\mathbb{A}_{f}\right)$ [Deligne 1979, 2.1.11]; for details see also [Ullmo and Yafaev 2013, Lemma 5.13].

Let $K_{0} \subset G\left(\mathbb{A}_{f}\right)$ be a neat compact open subgroup. If $K \subset K_{0}$ is an open subgroup, the induced morphism $p_{K, K_{0}}: \mathrm{Sh}_{K}(G, X) \rightarrow \mathrm{Sh}_{K_{0}}(G, X)$ on Shimura varieties is finite étale. If, moreover, $K$ is normal in $K_{0}$, this morphism is Galois 
with group $K_{0} / K$. Fix a point $s \in \operatorname{Sh}(G, X)$ and for every open subgroup $K \subset K_{0}$, let $s[K]$ denote the image of $s$ in $\operatorname{Sh}_{K}(G, X)$, and let $S[K, s] \subset \operatorname{Sh}_{K}(G, X)$ denote the geometrically connected component of $s[K]$ and $E[K]:=E[K](G, X)$ its field of definition (a finite abelian extension of the reflex field $E:=E(G, X)$ ). Let $\tilde{S}[K, s] \subset \operatorname{Sh}_{K}(G, X)_{E\left[K_{0}\right]}$ be the connected component of $s[K]$ in $\operatorname{Sh}_{K}(G, X)_{E\left[K_{0}\right]}$ (explicitly, $\tilde{S}[K, s]$ is the union of the $\operatorname{Gal}\left(E[K] \mid E\left[K_{0}\right]\right.$ )-conjugate of $S[K, s]$ ). The tower of (connected) pointed Galois covers

$$
p_{K, K_{0}, s}:(\tilde{S}[K, s], s[K]) \rightarrow\left(S\left[K_{0}, s\right], s\left[K_{0}\right]\right)
$$

corresponds to a projective system of continuous group morphisms

$$
\pi_{1}\left(S\left[K_{0}, s\right], \bar{s}\left[K_{0}\right]\right) \rightarrow \operatorname{Aut}\left(p_{K, K_{0}, s}\right) \subset K_{0} / K .
$$

As the intersection of all open normal subgroups $K \subset K_{0}$ is trivial, passing to the limit we obtain a continuous group morphism

$$
\rho\left[K_{0}, s\right]: \pi_{1}\left(S\left[K_{0}, s\right], \bar{s}\left[K_{0}\right]\right) \rightarrow \underset{K}{\lim } K_{0} / K=K_{0} .
$$

By construction, this morphism satisfies the following properties.

4.1. Functoriality. Let $f:\left(G_{2}, X_{2}\right) \rightarrow\left(G_{1}, X_{1}\right)$ be a morphism of Shimura data and $K_{i} \subset G_{i}\left(\mathbb{A}_{f}\right), i=1,2$ neat compact open subgroups such that $f\left(K_{2}\right) \subset K_{1}$; these induces morphisms of Shimura varieties $\operatorname{Sh}\left(G_{2}, X_{2}\right) \rightarrow \operatorname{Sh}\left(G_{1}, X_{1}\right)_{E_{2}}$ and $\mathrm{Sh}_{K_{2}}\left(G_{2}, X_{2}\right) \rightarrow \operatorname{Sh}_{K_{1}}\left(G_{1}, X_{1}\right)_{E_{2}}$ over the reflex field $E_{2}:=E\left(G_{2}, X_{2}\right)$. Fix $s_{2} \in \operatorname{Sh}\left(G_{2}, X_{2}\right)$ and set $s_{1}:=f\left(s_{2}\right) \in \operatorname{Sh}\left(G_{1}, X_{1}\right)$. Then the following diagram commutes:

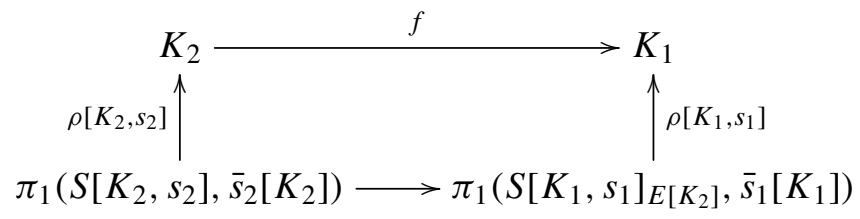

where, as above, $E\left[K_{2}\right]:=E\left[K_{2}\right]\left(G_{2}, X_{2}\right)$ denotes the field of definition of the geometrically connected component $S\left[K_{2}, s_{2}\right]$ of $s_{2}\left[K_{2}\right]$ in $\mathrm{Sh}_{K_{2}}\left(G_{2}, X_{2}\right)$.

4.2. Change of connected component. Assume the $\mathbb{C}$-valued point

$$
s_{\mathbb{C}} \in \operatorname{Sh}(G, X)(\mathbb{C})=G(\mathbb{Q}) \backslash X \times G\left(\mathbb{A}_{f}\right)
$$

corresponding to $s$ is of the form $s_{\mathbb{C}}=G(\mathbb{Q})(x, 1)$, let $a \in G\left(\mathbb{A}_{f}\right)$ and write $s a \in \operatorname{Sh}(G, X)$ for the point corresponding to $s_{\mathbb{C}} a=G(\mathbb{Q})(x, a)$. For a neat compact open subgroup $K \subset G\left(\mathbb{A}_{f}\right)$, write $K^{a}:=K \cap a K a^{-1}$. As the Heckeoperator $-a: \operatorname{Sh}(G, X) \stackrel{\sim}{\rightarrow} \operatorname{Sh}(G, X)$ is defined over the reflex field, the following diagram commutes: 


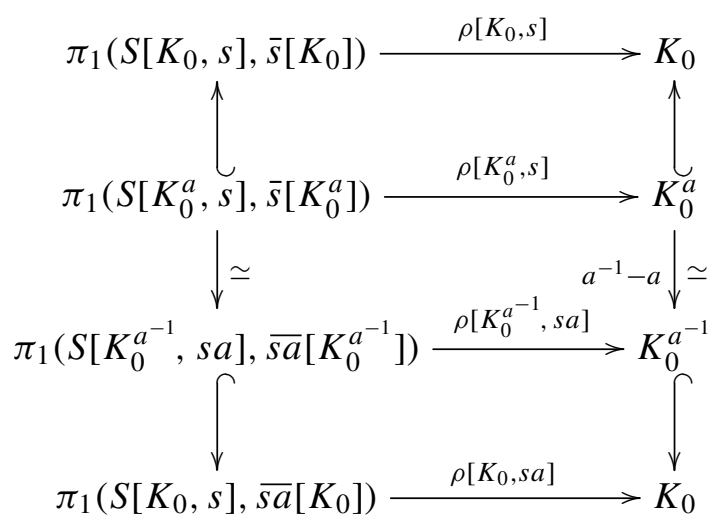

where the downwards vertical arrow on the left is induced by the isomorphism $-a: S\left[K_{0}^{a}, s\right] \stackrel{\sim}{\rightarrow} S\left[K_{0}^{a^{-1}}, s a\right]$ (mapping $s\left[K_{0}^{a}\right]$ to $s a\left[K_{0}^{a^{-1}}\right]$ ), and the upper and lower left arrows are open embeddings.

Let $X^{+} \subset X$ denote the connected component of $x \in X$ and $G(\mathbb{Q})_{+} \subset G(\mathbb{Q})$ the stabilizer of $X^{+}$in $G(\mathbb{Q})$. Given an open subgroup $K \subset K_{0}$, write $\Gamma:=G(\mathbb{Q})_{+} \cap K$ and $\Gamma_{0}:=G(\mathbb{Q})_{+} \cap K_{0}$. Then $\tilde{S}[K, s]_{E_{K}}$ splits into a disjoint union of geometrically connected components isomorphic to the connected component $\operatorname{Sh}_{\Gamma}\left(G, X^{+}\right)$of

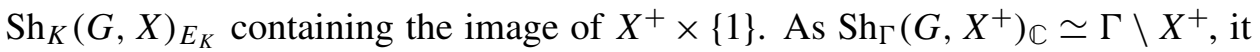
follows that $\rho_{K_{0}, s}: \pi_{1}\left(S_{K_{0}, s}, \bar{s}_{K_{0}}\right) \rightarrow K_{0}$ restricts to a surjective continuous group morphism

$$
\rho\left[K_{0}, s\right]: \pi_{1}\left(S\left[K_{0}, s\right]_{\bar{E}}, \bar{s}\left[K_{0}\right]\right) \rightarrow \Gamma_{0}^{-} .
$$

Here we implicitly identify the closure $\Gamma_{0}^{-}$of $\Gamma_{0}$ in $G\left(\mathbb{A}_{f}\right)$ with $\lim _{\leftarrow} \Gamma_{0} / \Gamma$, where the projective limit is taken over all normal congruence subgroups of $\Gamma_{0}$.

Actually, as $E[K]$ is contained in the maximal abelian extension $E^{\mathrm{ab}}$ of the reflex field $E$, the above shows that $\rho\left[K_{0}, s\right]: \pi_{1}\left(S\left[K_{0}, s\right], \bar{s}\left[K_{0}\right]\right) \rightarrow K_{0}$ already restricts to a surjective continuous group morphism

$$
\rho\left[K_{0}, s\right]: \pi_{1}\left(S\left[K_{0}, s\right]_{\left.E^{\mathrm{ab}}, \bar{s}\left[K_{0}\right]\right) \rightarrow \Gamma_{0}^{-},}\right.
$$

which is completely determined by the tower of connected étale covers over $E^{\mathrm{ab}}$ :

$$
p_{\Gamma, \Gamma_{0}}: \operatorname{Sh}_{\Gamma}\left(G, X^{+}\right)_{E^{\mathrm{ab}}} \rightarrow \operatorname{Sh}_{\Gamma_{0}}\left(G, X^{+}\right)_{E^{\mathrm{ab}}},
$$

where $\Gamma$ describes all normal congruence subgroups of $\Gamma_{0}$.

4.3. Change of base point. Let $s, s^{\prime} \in \operatorname{Sh}(G, X)$ be two points lying in the same geometrically connected component; write $S\left[K_{0}, s\right]=S\left[K_{0}, s^{\prime}\right]=: S\left[K_{0}\right]$. Then every étale path $\alpha: \bar{s}\left[K_{0}\right] \rightarrow \bar{s}^{\prime}\left[K_{0}\right]$ mapping $\bar{s}$ to $\bar{s}^{\prime}$ induces an isomorphism of profinite groups $\alpha: \pi_{1}\left(S\left[K_{0}\right], \bar{s}\left[K_{0}\right]\right) \stackrel{\sim}{\rightarrow} \pi_{1}\left(S\left[K_{0}\right], \bar{s}^{\prime}\left[K_{0}\right]\right)$, which makes the following diagram commute: 


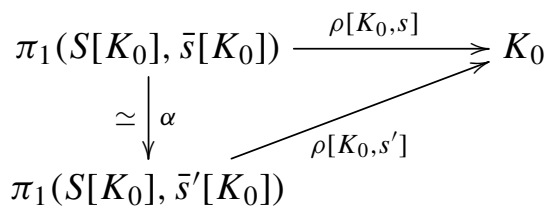

4.4. Galois-generic points. For $s \in \operatorname{Sh}(G, X)$ the following assertions are equivalent (see 3.2.2 and 3.2.5):

(1) There exists a neat compact open subgroup $K \subset G\left(\mathbb{A}_{f}\right)$ such that $s[K] \in S[K, s]$ is $(\ell-)$ Galois-generic with respect to $\rho[K, s]: \pi_{1}(S[K, s], \bar{s}[K]) \rightarrow K \subset G\left(\mathbb{A}_{f}\right)$.

(2) For every neat compact open subgroup $K \subset G\left(\mathbb{A}_{f}\right), s[K] \in S[K, s]$ is $(\ell-)$ Galoisgeneric with respect to $\rho[K, s]: \pi_{1}(S[K, s], \bar{s}[K]) \rightarrow K \subset G\left(\mathbb{A}_{f}\right)$.

In this case we say that $s \in \operatorname{Sh}(G, X)$ is $(\ell-)$ Galois-generic. In view of Section 4.2, we also see that for every $a \in G\left(\mathbb{A}_{f}\right), s \in \operatorname{Sh}(G, X)$ is Galois-generic if and only if $s a \in \operatorname{Sh}(G, X)$ is Galois-generic. So, in the following, we always assume that $s_{\mathbb{C}}=G(\mathbb{Q})(x, 1)$ and write $X^{+} \subset X$ for the connected component of $x$. In particular, with the notation of Section 4.2, $S\left[K_{0}, s\right]=\operatorname{Sh}_{\Gamma_{0}}\left(G, X^{+}\right)$and the restriction $\rho\left[K_{0}, s\right]: \pi_{1}\left(S\left[K_{0}, s\right]_{E^{\text {ab }}}, \bar{s}\left[K_{0}\right]\right) \rightarrow \Gamma_{0}^{-}$is completely determined by the tower of connected étale covers over $E^{a b}$,

$$
p_{\Gamma, \Gamma_{0}}: \operatorname{Sh}_{\Gamma}\left(G, X^{+}\right)_{E^{\mathrm{ab}}} \rightarrow \operatorname{Sh}_{\Gamma_{0}}\left(G, X^{+}\right)_{E^{\mathrm{ab}}} .
$$

Also, in view of Section 4.3, we shall omit the reference to $s$ in the notation (e.g., write $S\left[K_{0}\right], \rho\left[K_{0}\right], \pi_{1}\left(S\left[K_{0}\right]\right)$ instead of $S\left[K_{0}, s\right], \rho\left[K_{0}, s\right], \pi_{1}\left(S\left[K_{0}, s\right], \bar{s}\left[K_{0}\right]\right)$, etc.) unless it plays a part in the discussion.

4.5. Adelic representation attached to Siegel Shimura varieties. Let $\left(\mathrm{GSp}_{2 g}, X\right)$ denote the Siegel Shimura datum [Deligne 1971, Exemple 1.6]. Using the moduli description of the attached Shimura variety [Deligne 1971, Exemple 4.16], one easily shows that for a neat compact open subgroup $K_{0} \subset \mathrm{GSp}_{2 g}\left(\mathbb{A}_{f}\right)$ and geometrically connected component $S\left[K_{0}\right] \subset \mathrm{Sh}_{K_{0}}\left(\mathrm{GSp}_{2 g}, X\right)$, the corresponding adelic representation $\rho\left[K_{0}\right]: \pi_{1}\left(S\left[K_{0}\right]\right) \rightarrow K \subset G\left(\mathbb{A}_{f}\right)$ identifies with the representation $\rho: \pi_{1}\left(S\left[K_{0}\right]\right) \rightarrow \operatorname{GL}\left(T(A)_{\bar{\eta}}\right)$ on the adelic Tate module of the universal abelian scheme $A \rightarrow S\left[K_{0}\right]$. (See also [Ullmo and Yafaev 2013].)

\section{Group-theoretical preliminaries}

In this section, we gather technical group-theoretical results about the adelic closure of arithmetic subgroups of semisimple algebraic groups. These will be used in the proof of Theorem A to deduce the case of Shimura data of abelian type from the case of Shimura data of Hodge type. 
Let $G$ be a group. Recall that two subgroups $K, K^{\prime} \subset G$ are said to be commensurable if $K \cap K^{\prime}$ is of finite index in both $K$ and $K^{\prime}$. Commensurability is an equivalence relation, which we denote by $\equiv$, on the set of subgroups of $G$.

For an algebraic group $G$ over $\mathbb{Q}$, a faithful $\mathbb{Q}$-linear representation $G \hookrightarrow \operatorname{GL}(V)$ and a $\mathbb{Z}$-lattice $L \subset V$, write $G_{L}$ for the subgroups of elements $g \in G(\mathbb{Q})$ stabilizing $L$. If $G \hookrightarrow \mathrm{GL}(V), G \hookrightarrow \mathrm{GL}\left(V^{\prime}\right)$ are two faithful $\mathbb{Q}$-linear representations and $L \subset V$, $L^{\prime} \subset V^{\prime}$ are $\mathbb{Z}$-lattices then $G_{L} \equiv G_{L^{\prime}} \subset G$. Thus the class of commensurability of $G_{L}$ does not depend on the choices of $G \hookrightarrow \operatorname{GL}(V)$ and $L \subset V$; the groups in this class are the arithmetic subgroups of $G$. Arithmetic subgroups have the following properties.

Fact 5.1. (1) For an algebraic group $G$ over $\mathbb{Q}$, a faithful $\mathbb{Q}$-linear representation $G \hookrightarrow \mathrm{GL}(V)$ and an arithmetic subgroup $\Gamma \subset G(\mathbb{Q})$, there exists $a$-invariant $\mathbb{Z}$-lattice $L \subset V$.

(2) For a surjective morphism $f: G_{2} \rightarrow G_{1}$ of algebraic groups over $\mathbb{Q}$ and an arithmetic subgroup $\Gamma \subset G_{2}(\mathbb{Q})$, the subgroup $f(\Gamma) \subset G_{1}(\mathbb{Q})$ is again arithmetic.

Let $G$ be a semisimple algebraic group over $\mathbb{Q}$ and $\Gamma \subset G(\mathbb{Q})$ an arithmetic subgroup. Then:

(3) $\Gamma$ is finitely presented as an abstract group.

(4) If, furthermore, $G$ is of noncompact type then $\Gamma$ is Zariski-dense in $G$.

An algebraic group $G$ over $\mathbb{Q}$ is said to be of compact type if $G(\mathbb{R})$ is compact [Platonov and Rapinchuk 1994, Definition p. 205]. A semisimple algebraic group over $\mathbb{Q}$ is said to be of noncompact type if none of its simple factors is of compact type.

Proof. For assertions (1), (2) and (3), see [Platonov and Rapinchuk 1994, Proposition 4.2, Theorems 4.1 and 4.2], respectively. Assertion (4) is the Borel density theorem [Borel 1966]; see also [Platonov and Rapinchuk 1994, Theorem 4.10].

For an algebraic group $G$ over $\mathbb{Q}$ and a subgroup $\Gamma \subset G(\mathbb{Q})$, let $\Gamma^{-} \subset G\left(\mathbb{A}_{f}\right)$ denote the adelic closure of $\Gamma$ in $G\left(\mathbb{A}_{f}\right)$.

Lemma 5.2. If $\Gamma \subset G(\mathbb{Q})$ is an arithmetic subgroup, then $\Gamma^{-}$is profinite and the collection of subgroups $\Gamma^{\prime-}$, for $\Gamma^{\prime} \subset \Gamma$ a normal subgroup of finite index, is a fundamental system of open neighborhoods of 1 in $\Gamma^{-}$.

For an algebraic subgroup $G \subset \mathrm{GL}_{n, \mathbb{Q}}$ and a ring $A$ of characteristic 0 , write $G(A):=G(\mathbb{Q}) \cap \mathrm{GL}_{n}(A)$.

Proof. Let $G \hookrightarrow \operatorname{GL}(V)$ be a faithful $\mathbb{Q}$-rational representation of $G$ and $L \subset V$ a $\Gamma$-invariant $\mathbb{Z}$-lattice (see Fact 5.1(1)). Fixing a $\mathbb{Z}$-basis of $L$ we get an isomorphism $\mathrm{GL}(V) \simeq \mathrm{GL}_{n, \mathbb{Q}}$ such that $\Gamma \subset G(\mathbb{Z})$. Then $\Gamma^{-}$is a closed subgroup of the profinite 
group $\prod_{\ell} G\left(\mathbb{Z}_{\ell}\right)$, hence is profinite. In particular, a subgroup of $\Gamma^{-}$is open if and only if it is closed of finite index in $\Gamma^{-}$. As for any subgroup $\Gamma^{\prime} \subset \Gamma$ of finite index, $\Gamma^{\prime-} \subset \Gamma^{-}$is a closed subgroup of finite index $\leq\left[\Gamma: \Gamma^{\prime}\right]$, one already sees that the $\Gamma^{\prime-}$, with $\Gamma^{\prime} \subset \Gamma$ of finite index, are open neighborhoods of 1 in $\Gamma^{-}$. Moreover, for every open subgroup $U \subset \Gamma^{-}$, the intersection $\Gamma \cap U \subset \Gamma$ has finite index $\leq\left[\Gamma^{-}: U\right]$ and, by construction, $(\Gamma \cap U)^{-} \subset U^{-}=U$. Eventually, if $\Gamma^{\prime} \subset \Gamma$ is a subgroup of finite index, then

$$
\bigcap_{\gamma \in \Gamma} \gamma \Gamma^{\prime} \gamma^{-1} \subset \Gamma^{\prime}
$$

is normal and again of finite index $\leq\left[\Gamma: \Gamma^{\prime}\right]$ ! in $\Gamma$.

For a closed subgroup $U \subset \mathrm{GL}_{n}\left(\mathbb{Z}_{\ell}\right)$, let $U^{+} \subset U$ denote the (normal) subgroup generated by the $\ell$-Sylow subgroups of $U$.

Lemma 5.3. Let $G \subset \mathrm{GL}_{n, \mathbb{Q}}$ be an algebraic subgroup and $U \subset G\left(\mathbb{A}_{f}\right)$ a closed subgroup such that $U_{\ell} \subset G\left(\mathbb{Z}_{\ell}\right)$ for $\ell \gg 0$. Assume that $U_{\ell}=U_{\ell}^{+}$for $\ell \gg 0$. Then there exists an open subgroup $U^{\prime} \subset U$ such that $U^{\prime}=\prod_{\ell} U_{\ell}^{\prime}$.

Proof. This follows from a combination of results about almost- $\ell$ independency in the sense of Serre [2013]. More precisely, given an infinite set of primes $L$, a family $G_{\ell}, \ell \in L$, of $\ell$-adic Lie groups and a profinite group $\Delta \subset \prod_{\ell} G_{\ell}$, one says that $\Delta$ is $\ell$-independent (as a subgroup of $\prod_{\ell} G_{\ell}$ ) if $\Delta=\prod_{\ell} \Delta_{\ell}$, and that $\Delta$ is almost $\ell$-independent if there exists an open subgroup $\Delta^{\prime} \subset \Delta$ which is $\ell$-independent as a subgroup of $\prod_{\ell} G_{\ell}$. With these definitions, the following hold:

(1) [Serre 2013, Lemma 1] If for $\ell \neq \ell^{\prime}$ no simple quotient of $\Delta_{\ell}$ is isomorphic to a simple quotient of $\Delta_{\ell^{\prime}}$, then $\Delta \subset \prod_{\ell} G_{\ell}$ is $\ell$-independent.

(2) [Serre 2013, Lemma 3] If there exists a finite subset $F \subset L$ such that the image of $\Delta$ in $\prod_{L \backslash F} G_{\ell}$ is almost $\ell$-independent, then $\Delta \subset \prod_{\ell} G_{\ell}$ is almost $\ell$-independent.

For every prime $\ell$, let $\Sigma_{\ell}$ denote the set of all (isomorphism classes of) finite groups which are either a simple group of Lie type in characteristic $\ell$ (see [Serre $2013, \S 6.1])$ or $\mathbb{Z} / \ell$.

(3) [Serre 2013, Theorem 4] Every finite simple subquotient of $\mathrm{GL}_{n}\left(\mathbb{Z}_{\ell}\right)$ of order divisible by $\ell$ is in $\Sigma_{\ell}$ for $\ell \gg 0$ (depending on $n$ ).

(4) [Serre 2013, Theorem 5] For $\ell, \ell^{\prime} \geq 5$ with $\ell \neq \ell^{\prime}$, one has $\Sigma_{\ell} \cap \Sigma_{\ell^{\prime}}=\varnothing$.

From (2), it is enough to show that the image $U_{L}$ of $U$ in $\prod_{\ell \in L} G\left(\mathbb{Q}_{\ell}\right)$ is almost $\ell$-independent for a set $L$ containing all but finitely many primes. In particular, one may assume that $U_{\ell} \subset G\left(\mathbb{Z}_{\ell}\right)$, that $U_{\ell}=U_{\ell}^{+}$for every $\ell \in L$ and that the conclusions of (3) and (4) hold for $\ell \in L$ and $\ell \neq \ell^{\prime} \in L$, respectively. As every simple quotient 
of $U_{\ell}^{\#}\left(=U_{\ell}\right)$ is in $\Sigma_{\ell}$ for $\ell \in L$, (4) and (1) show that $U_{L}$ is $\ell$-independent, as requested.

Theorem 5.4. Let $G$ be a semisimple algebraic group over $\mathbb{Q}$. Let $\Gamma \subset G(\mathbb{Q})$ be an arithmetic subgroup. Then every open subgroup $U$ of $\Gamma^{-} \subset G\left(\mathbb{A}_{f}\right)$ has finite abelianization.

Proof. Fix an embedding $G \hookrightarrow \mathrm{GL}_{n, \mathbb{Q}}$. We let again $G$ denote the Zariski closure of $G$ in $\mathrm{GL}_{n, \mathbb{Z}}$; this is a semisimple group over some nonempty open subscheme of $\operatorname{spec}(\mathbb{Z})$.

- Reduction to the case where $U$ is of the form $\Gamma^{-}$for some normal arithmetic subgroup $\Gamma \subset G(\mathbb{Z})$. From Lemma 5.2, there exists a subgroup $\Gamma^{\prime} \subset \Gamma$ normal, of finite index in $\Gamma$ and such that $\Gamma^{\prime-} \subset U$. From the finiteness of $U / \Gamma^{\prime-}$ and the exact sequence

$$
\left(\Gamma^{\prime-}\right)^{\mathrm{ab}} \rightarrow U^{\mathrm{ab}} \rightarrow\left(U / \Gamma^{\prime-}\right)^{\mathrm{ab}} \rightarrow 0,
$$

it is enough to perform the proof for $\Gamma^{--}$; that is, we may assume $U$ is of the form $\Gamma^{-}$for some arithmetic subgroup $\Gamma \subset G(\mathbb{Q})$. Next, as $\Gamma$ is finitely generated as an abstract group (Fact 5.1(3)), it has only finitely many subgroups of bounded index $\leq[\Gamma: G(\mathbb{Z}) \cap \Gamma]$. In particular, the group

$$
\Delta:=\bigcap_{g \in G(\mathbb{Z}) \Gamma} g G(\mathbb{Z}) \cap \Gamma g^{-1} \subset G(\mathbb{Q})
$$

is again an arithmetic subgroup, contained and normal in both $\Gamma$ and $G(\mathbb{Z})$. So the conclusion follows from the finiteness of $\Gamma / \Delta$ and the exact sequence

$$
\Delta^{-\mathrm{ab}} \rightarrow \Gamma^{-\mathrm{ab}} \rightarrow\left(\Gamma^{-} / \Delta^{-}\right)^{\mathrm{ab}} \rightarrow 0 .
$$

- Reduction to the case where $G$ is of noncompact type. Let $G^{\mathrm{nc}} \subset G$ denote the largest (normal) algebraic subgroup of $G$ of noncompact type, and $p: G \rightarrow G / G^{\mathrm{nc}}$ the canonical projection. Write

$$
\Gamma^{\mathrm{nc}}:=\Gamma \cap G^{\mathrm{nc}}(\mathbb{Q}) \subset G^{\mathrm{nc}}(\mathbb{Q}) .
$$

As $p(\Gamma) \subset G / G^{\mathrm{nc}}(\mathbb{Q})$ is again an arithmetic subgroup (Fact 5.1(3)) and $G / G^{\mathrm{nc}}$ is of compact type, $p(\Gamma)$ is finite; in particular, $\Gamma^{-} / \Gamma^{\mathrm{nc}-}$ is finite. Also, by construction, $\Gamma^{\mathrm{nc}}$ is contained and normal in $G^{\mathrm{nc}}(\mathbb{Z})$ and $\left[G^{\mathrm{nc}}(\mathbb{Z}): \Gamma^{\mathrm{nc}}\right] \leq[G(\mathbb{Z}): \Gamma]$, which shows that $\Gamma^{\mathrm{nc}} \subset G^{\mathrm{nc}}(\mathbb{Q})$ is again an arithmetic subgroup. Thus the conclusion follows from the finiteness of $\Gamma^{-} / \Gamma^{\mathrm{nc}-}$ and the exact sequence

$$
\Gamma^{\mathrm{nc}-\mathrm{ab}} \rightarrow \Gamma^{-\mathrm{ab}} \rightarrow\left(\Gamma^{-} / \Gamma^{\mathrm{nc}-}\right)^{\mathrm{ab}} \rightarrow 0 .
$$

- Reduction to the case where $\Gamma_{\ell}^{-}=G\left(\mathbb{Z}_{\ell}\right)^{+}$for $\ell \gg 0$. For a prime $\ell$, let $p_{\ell}: \mathrm{GL}_{n}(\mathbb{Z}) \rightarrow \mathrm{GL}_{n}\left(\mathbb{F}_{\ell}\right)$ denote the reduction modulo- $\ell$ morphism. Then, as 
$\Gamma$ is finitely generated as an abstract group (Fact 5.1(3)) and Zariski-dense in $G$ (Fact 5.1(4)), we have

$$
G\left(\mathbb{F}_{\ell}\right)^{+} \subset p_{\ell}(\Gamma) \subset G\left(\mathbb{F}_{\ell}\right)
$$

for $\ell \gg 0$ depending only on $n$ [Nori 1987, Theorem 5.1]. Here $G\left(\mathbb{F}_{\ell}\right)^{+} \subset G\left(\mathbb{F}_{\ell}\right)$ denotes the (normal) subgroup generated by the order- $\ell$ elements in $G\left(\mathbb{F}_{\ell}\right)$ (or, equivalently, the $\ell$-Sylow subgroups as soon as $\ell>n$ ). As $G$ is semisimple, $G\left(\mathbb{F}_{\ell}\right) / G\left(\mathbb{F}_{\ell}\right)^{+}$is abelian of order $\leq 2^{n-1}$. In particular, there exists an integer $N \geq 1$ such that for every prime $\ell$ the subgroup

$$
\Delta[\ell]:=p_{\ell}^{-1}\left(G\left(\mathbb{F}_{\ell}\right)^{+}\right) \cap \Gamma \subset \Gamma
$$

is normal and of index $\leq N$ in $\Gamma$. As $\Gamma$ is finitely generated, it has only finitely many subgroups of index $\leq N$. So

$$
\Delta:=\bigcap_{\ell} \Delta[\ell] \subset \Gamma
$$

is again a subgroup normal and of finite index in $\Gamma$. For $\ell>[\Gamma: \Delta]$ and $\ell \gg 0$ such that $G\left(\mathbb{F}_{\ell}\right)^{+} \subset \bar{\Gamma}_{\ell}$, we have

$$
\left[G\left(\mathbb{F}_{\ell}\right)^{+}: p_{\ell}(\Delta)\right]=\left[p_{\ell}(\Delta[\ell]): p_{\ell}(\Delta)\right] \leq[\Delta[\ell]: \Delta] \leq[\Gamma: \Delta]<\ell .
$$

As $G\left(\mathbb{F}_{\ell}\right)^{+}$is generated by its order- $\ell$ elements, this forces $p_{\ell}(\Delta)=G\left(\mathbb{F}_{\ell}\right)^{+}$. Then the finiteness of $\Gamma^{-} / \Delta^{-}$and the exact sequence

$$
\Delta^{-\mathrm{ab}} \rightarrow \Gamma^{-\mathrm{ab}} \rightarrow\left(\Gamma^{-} / \Delta^{-}\right)^{\mathrm{ab}} \rightarrow 0
$$

show that it is enough to prove that $\Delta^{- \text {ab }}$ is finite. That is, without loss of generality, we may replace $\Gamma$ with $\Delta$, and hence assume that $p_{\ell}(\Gamma)=G\left(\mathbb{F}_{\ell}\right)^{+}$for $\ell \gg 0$. As $p_{\ell}^{-}\left(\Gamma_{\ell}^{-}\right)=p_{\ell}\left(\Gamma_{\ell}\right)=G\left(\mathbb{F}_{\ell}\right)^{+}$and $\operatorname{ker}\left(p_{\ell}^{-}\right) \subset G\left(\mathbb{Z}_{\ell}\right)$ is a pro- $\ell$ group, this implies $\Gamma_{\ell}^{-}=\left(\Gamma_{\ell}^{-}\right)^{+}$, and hence $\Gamma_{\ell}^{-} \subset G\left(\mathbb{Z}_{\ell}\right)^{+}$. Assume also that $\ell \gg 0$, so that $G_{\mathbb{Z}_{\ell}}$ is semisimple over $\mathbb{Z}_{\ell}$. Then the reduction-modulo- $\ell$ morphism $p_{\ell}^{-}: G\left(\mathbb{Z}_{\ell}\right) \rightarrow G\left(\mathbb{F}_{\ell}\right)$ is surjective and, up to increasing $\ell$, we may assume that the induced surjective morphism

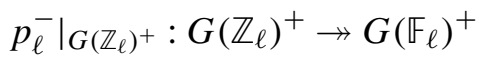

is Frattini [Cadoret 2015, Fact 2.4, Lemma 2.5]; that is, $G\left(\mathbb{Z}_{\ell}\right)^{+}$is the unique closed subgroup $X \subset G\left(\mathbb{Z}_{\ell}\right)^{+}$mapping subjectively onto $G\left(\mathbb{F}_{\ell}\right)^{+}$. This shows that $\Gamma_{\ell}^{-}=G\left(\mathbb{Z}_{\ell}\right)^{+}$.

- End of the proof. As $\Gamma_{\ell}^{-}=\left(\Gamma_{\ell}^{-}\right)^{+}$for $\ell \gg 0$, there exists (Lemma 5.3) an open subgroup $U \subset \Gamma^{-}$such that $U=\prod_{\ell} U_{\ell}$. As $\Gamma^{-}$is profinite, $U$ is of finite index in $\Gamma^{-}$, and thus $\left[\Gamma_{\ell}^{-}: U_{\ell}\right] \leq\left[\Gamma^{-}: U\right]$ for every $\ell$. On the other hand, as $\Gamma_{\ell}^{-}=\left(\Gamma_{\ell}^{-}\right)^{+}$, 
all subgroups of $\Gamma_{\ell}^{-}$have index $\geq \ell$ in $\Gamma_{\ell}^{-}$. This forces $U_{\ell}=\Gamma_{\ell}^{-}$for $\ell \gg 0$. Also, up to replacing $U_{\ell}$ by

$$
\bigcap_{\gamma_{\ell} \in \Gamma_{\ell}^{-}} \gamma_{\ell} U_{\ell} \gamma_{\ell}^{-1}
$$

for small $\ell$, we may assume that $U$ is normal in $\prod_{\ell} \Gamma_{\ell}^{-}$(hence a fortiori in $\Gamma^{-}$). Then the exact sequence

$$
U^{\mathrm{ab}} \rightarrow \Gamma^{-\mathrm{ab}} \rightarrow\left(\Gamma^{-} / U\right)^{\mathrm{ab}} \rightarrow 0
$$

shows that it is enough to prove that $U^{\mathrm{ab}}=\prod_{\ell} U_{\ell}^{\mathrm{ab}}$ is finite; equivalently,

(1) $U_{\ell}^{\mathrm{ab}}$ is finite for every $\ell$;

(2) $U_{\ell}^{\mathrm{ab}}=0$ for $\ell \gg 0$ (or, equivalently, $\left(G\left(Z_{\ell}\right)^{+}\right)^{\mathrm{ab}}=0$ for $\ell \gg 0$ ).

Proof of (1): If (1) were false, $U_{\ell}$ would have an infinite abelian quotient $U_{\ell} \rightarrow Z_{\ell}$. As $U_{\ell}$ is an $\ell$-adic Lie group, so is $Z_{\ell}$ and $Z_{\ell}$ has dimension $\geq 1$ as an $\ell$-adic Lie group. From exactness of the Lie algebra functor on the category of $\ell$-adic Lie groups, we obtain a surjective morphism of Lie algebras

$$
\operatorname{Lie}\left(U_{\ell}\right) \rightarrow \operatorname{Lie}\left(Z_{\ell}\right) .
$$

On the other hand, since $G$ is semisimple, we also have $\operatorname{Lie}\left(U_{\ell}\right)=\operatorname{Lie}\left(\Gamma_{\ell}^{-}\right)=$ $\operatorname{Lie}(G) \otimes \mathbb{Q}_{\ell}$, which has no abelian quotient as a Lie algebra.

Proof of (2): Since, as noted above,

$$
\left.p_{\ell}^{-}\right|_{G\left(\mathbb{Z}_{\ell}\right)^{+}}: G\left(\mathbb{Z}_{\ell}\right)^{+} \rightarrow G\left(\mathbb{F}_{\ell}\right)^{+}
$$

is Frattini and $\left[G\left(\mathbb{Z}_{\ell}\right)^{+}, G\left(\mathbb{Z}_{\ell}\right)^{+}\right]^{-}$maps surjectively onto $\left[G\left(\mathbb{F}_{\ell}\right)^{+}, G\left(\mathbb{F}_{\ell}\right)^{+}\right]$, it is enough to show that $\left[G\left(\mathbb{F}_{\ell}\right)^{+}, G\left(\mathbb{F}_{\ell}\right)^{+}\right]=G\left(\mathbb{F}_{\ell}\right)^{+}$; that is, $G\left(\mathbb{F}_{\ell}\right)^{+ \text {ab }}=0$. This fact is probably well-known to specialists. However, for lack of a suitable reference, we sketch the argument.

Without loss of generality, we may assume $G$ is semisimple over $\mathbb{Z}$. Then, for $\ell \gg 0, G_{\mathbb{F}_{\ell}}$ coincides with the algebraic envelope (in the sense of Nori [1987]) $G\left(\mathbb{F}_{\ell}\right)^{\sim} \subset \mathrm{GL}_{n, \mathbb{F}_{\ell}}$ of $G\left(\mathbb{F}_{\ell}\right)$ in $\mathrm{GL}_{n, \mathbb{F}_{\ell}}$. More precisely, if $P_{1}, \ldots, P_{r} \in \mathbb{Z}\left[X_{i, j}, Y\right]$ are polynomial equations defining $G \subset \mathrm{GL}_{n, \mathbb{Z}} \simeq M_{n}(\mathbb{Z})[1 / \mathrm{det}] \subset \mathbb{Z}^{n^{2}+1}$ for every $g \in G\left(\mathbb{F}_{\ell}\right)$ of order $\ell$, the polynomial $P_{i, g}(T):=P_{i}(\exp (T \log (g))) \in \mathbb{F}_{\ell}[T]$ has degree bounded from above by a constant $\delta_{i}$ independent of $\ell$. As $P_{i, g}(T)$ has at least $\ell$ distinct roots, this forces $P_{i, g}(T)=0$ as soon as $\ell>\delta_{i}$. In other words, $G_{\mathbb{F}_{\ell}}$ contains the one-parameter subgroup

$$
\begin{aligned}
e_{g}: \mathbb{A}_{\mathbb{F}_{\ell}}^{1} & \rightarrow \mathrm{GL}_{n, \mathbb{F}_{\ell}}, \\
t & \mapsto \exp (t \log (g)) .
\end{aligned}
$$


So, for $\ell>\max \left\{\delta_{1}, \ldots, \delta_{r}\right\}, G_{\mathbb{F}_{\ell}}$ contains $G\left(\mathbb{F}_{\ell}\right)^{\sim}$. On the other hand [Nori 1987, Theorem B], for $\ell \gg 0$,

$$
G\left(\mathbb{F}_{\ell}\right)^{\sim}\left(\mathbb{F}_{\ell}\right)^{+}=G\left(\mathbb{F}_{\ell}\right)^{+} .
$$

As $\left[G\left(\mathbb{F}_{\ell}\right)^{\sim}\left(\mathbb{F}_{\ell}\right): G\left(\mathbb{F}_{\ell}\right)^{\sim}\left(\mathbb{F}_{\ell}\right)^{+}\right] \leq 2^{n-1}$ (because $G\left(\mathbb{F}_{\ell}\right)^{\sim}$ is exponentially generated) and $\left[G\left(\mathbb{F}_{\ell}\right): G\left(\mathbb{F}_{\ell}\right)^{+}\right] \leq 2^{n-1}$ (because $G_{\mathbb{F}_{\ell}}$ is semisimple) [Nori 1987, Remark 3.6], $(\dagger)$ implies that $G\left(\mathbb{F}_{\ell}\right)^{\sim}$ and $G_{\mathbb{F}_{\ell}}$ have the same dimension [Nori 1987, Lemma 3.5]. As $G_{\mathbb{F}_{\ell}}$ is connected, this eventually yields $G\left(\mathbb{F}_{\ell}\right)^{\sim}=G_{\mathbb{F}_{\ell}}$ as claimed.

As $G\left(\mathbb{F}_{\ell}\right)^{\sim}=G_{\mathbb{F}_{\ell}}$ is semisimple, $G\left(\mathbb{F}_{\ell}\right)^{\sim}$ acts semisimply on $\mathbb{F}_{\ell}^{\oplus n}$ for $\ell \gg 0$ [Jantzen 1997, Proposition 3.2]. Since for $\ell \gg 0$ the $G\left(\mathbb{F}_{\ell}\right)^{\sim}$-submodules and $G\left(\mathbb{F}_{\ell}\right)^{+}$-submodules of $\mathbb{F}_{\ell}^{\oplus n}$ coincide, this in turn implies that $G\left(\mathbb{F}_{\ell}\right)^{+}$acts semisimply on $\mathbb{F}_{\ell}^{\oplus n}$. The conclusion then follows from [Cadoret and Tamagawa 2014, Lemma 3.4].

This concludes the proof of Theorem 5.4.

Lemma 5.5. Let $f: G_{2} \rightarrow G_{1}$ be an isogeny of connected semisimple algebraic groups over $\mathbb{Q}$. Let $\Gamma_{1} \subset G_{1}(\mathbb{Q})$ and $\Gamma_{2} \subset G_{2}(\mathbb{Q})$ be arithmetic subgroups such that $f\left(\Gamma_{2}\right) \subset \Gamma_{1}$. Then for every closed subgroup $\Delta \subset \Gamma_{2}^{-}$, the image $f(\Delta) \subset \Gamma_{1}^{-}$ is open if and only if $\Delta \subset \Gamma_{2}^{-}$is open.

Proof. This follows from Lemma 5.2 and the fact that a profinite group is compact and that its open subgroups are exactly its closed subgroups of finite index. As $f\left(\Gamma_{2}\right) \subset G_{1}(\mathbb{Q})$ is arithmetic, $f\left(\Gamma_{2}\right)$ and $\Gamma_{1}$ are commensurable. This implies that $f\left(\Gamma_{2}\right)^{-}$and $\Gamma_{1}^{-}$are commensurable as well, and hence that $f\left(\Gamma_{2}\right)^{-}$is open in $\Gamma_{1}^{-}$. But as $\Gamma_{2}^{-}$is compact, $f\left(\Gamma_{2}^{-}\right)=f\left(\Gamma_{2}\right)^{-}$. This shows that $f: \Gamma_{2}^{-} \rightarrow \Gamma_{1}^{-}$is a morphism of profinite groups with open image and finite kernel (since $f: G_{2} \rightarrow G_{1}$ is an isogeny). In particular, if $\Delta \subset \Gamma_{2}^{-}$has finite index then $f(\Delta) \subset \Gamma_{1}^{-}$also has finite index: $\left[\Gamma_{1}^{-}: f(\Delta)\right] \leq\left[\Gamma_{1}^{-}: f\left(\Gamma_{2}^{-}\right)\right]\left[\Gamma_{2}^{-}: \Delta\right]$. Conversely, if $f(\Delta) \subset \Gamma_{1}^{-}$has finite index then $\Delta \subset \Gamma_{2}^{-}$also has finite index:

$$
\left[\Gamma_{2}^{-}: \Delta\right] \leq|\operatorname{ker}(f)|\left[f\left(\Gamma_{2}^{-}\right): f(\Delta)\right] \leq|\operatorname{ker}(f)|\left[\Gamma_{1}^{-}: f(\Delta)\right] .
$$

\section{Galois-generic points for adelic representations attached to Shimura varieties}

Let $(G, X)$ be a Shimura datum.

6.1. Comparison with Pink's definition. Let $E:=E(G, X)$ denote the reflex field. A point $s \in \operatorname{Sh}(G, X)$ is Galois-generic in the sense of Pink [2005, Definition 6.3] if and only if $s_{E^{\mathrm{ab}}} \in \operatorname{Sh}(G, X)_{E^{\mathrm{ab}}}$ is Galois-generic in the sense of Section 4.4. With the notation of Lemma 3.2.4, one has $\tilde{E} \subset E^{\text {ab }}$, but in general the extension $\tilde{E} \subset E^{\mathrm{ab}}$ is not finite (when the reciprocity map describing the action of $\pi_{1}\left(E^{\mathrm{ab}}\right)$ on $\pi_{0}(\operatorname{Sh}(G, X))$ has infinite kernel). Still, the two notions of Galois-genericity 
coincide. More precisely, for $s \in \operatorname{Sh}(G, X)$ let $s^{\text {ad }} \in \operatorname{Sh}\left(G^{\text {ad }}, X^{\text {ad }}\right)$ denote its image by the canonical morphism

$$
\operatorname{Sh}(G, X) \rightarrow \operatorname{Sh}\left(G^{\mathrm{ad}}, X^{\mathrm{ad}}\right) .
$$

Proposition 6.1.1. For every $s \in \operatorname{Sh}(G, X)$, the following properties are equivalent:

(1) $s \in \operatorname{Sh}(G, X)$ is Galois-generic (resp. $\ell$-Galois-generic).

(2) $s_{E^{\mathrm{ab}}} \in \mathrm{Sh}(G, X)_{E^{\mathrm{ab}}}$ is Galois-generic (resp. $\ell$-Galois-generic).

(3) $s^{\mathrm{ad}} \in \operatorname{Sh}\left(G^{\mathrm{ad}}, X^{\mathrm{ad}}\right)$ is Galois-generic (resp. $\ell$-Galois-generic).

(4) $s_{E^{\mathrm{ab}}}^{\mathrm{ad}} \in \operatorname{Sh}\left(G^{\mathrm{ad}}, X^{\mathrm{ad}}\right)_{E^{\mathrm{ab}}}$ is Galois-generic (resp. $\ell$-Galois-generic).

Proof. The equivalences (1) $\Leftrightarrow$ (2) and (3) $\Leftrightarrow$ (4) follow from Lemma 3.2.4, from the fact that $\bar{\Pi}=\Gamma^{-} \subset G\left(\mathbb{A}_{f}\right)$ and from Theorem 5.4. For (2) $\Leftrightarrow$ (4), we may assume that the connected component of $s_{\mathbb{C}}$ is of the form $G(\mathbb{Q}) \backslash X^{+} \times\{1\}$ (see Section 4.4). Fix neat compact open subgroups $K \subset G\left(\mathbb{A}_{f}\right)$ and $K^{\text {ad }} \subset G^{\text {ad }}\left(\mathbb{A}_{f}\right)$ such that $p^{\text {ad }}(K) \subset K^{\text {ad }}$. Write $\Gamma:=K \cap G(\mathbb{Q})$ and $\Gamma^{\text {ad }}:=K^{\text {ad }} \cap G^{\text {ad }}(\mathbb{Q})$; we may assume $\Gamma \subset G^{\operatorname{der}}(\mathbb{Q})$. As $K$ is neat, $\Gamma$ maps injectively into $\Gamma^{\text {ad }}$. Then the geometrically connected component $S$ of $s[K]$ in $\operatorname{Sh}_{K}(G, X)$ is an étale cover of the geometrically connected component $S^{\mathrm{ad}}$ of $s^{\mathrm{ad}}\left[K^{\mathrm{ad}}\right]$ in $\operatorname{Sh}_{K^{\mathrm{ad}}}\left(G^{\mathrm{ad}}, X^{\mathrm{ad}}\right)$. The functoriality of adelic representations attached to Shimura varieties yields the following commutative diagram:

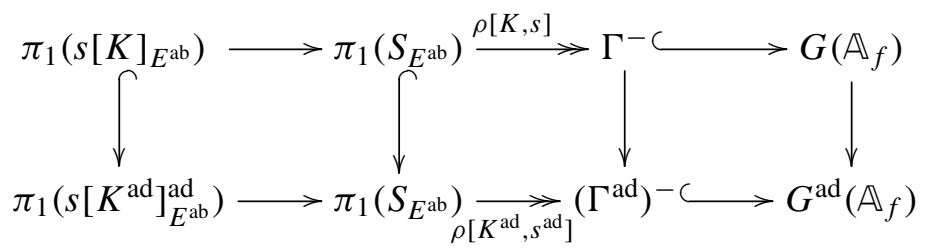

The conclusion then follows from the fact that the left vertical arrow has open image, and from Lemma 5.5 applied to the isogeny $G^{\mathrm{der}} \rightarrow G^{\text {ad }}$ and to $\Delta=\Pi_{s[K]_{E^{\mathrm{ab}}}} \subset \Gamma^{-}$. The proof for $\ell$-Galois-generic points is similar.

6.2. Galois-generic versus Hodge-generic points. We say that $x \in X$ is Hodgegeneric if $\operatorname{MT}(x)=G$. Let $X^{\text {hg }} \subset X$ denote the subset of Hodge-generic points. The set $X^{\mathrm{hg}}$ is analytically dense in $X$ and $G(\mathbb{Q}) X^{\mathrm{hg}}=X^{\mathrm{hg}}$. Let $K \subset G\left(\mathbb{A}_{f}\right)$ be a neat compact open subgroup. We say that $s \in \operatorname{Sh}(G, X)$ is Hodge-generic if $s_{\mathbb{C}} \in G(\mathbb{Q}) \backslash X^{\mathrm{hg}} \times G\left(\mathbb{A}_{f}\right) \subset \operatorname{Sh}(G, X)(\mathbb{C})$, and $s[K] \in \operatorname{Sh}_{K}(G, X)$ is Hodge-generic if $s[K]_{\mathbb{C}} \in G(\mathbb{Q}) \backslash X^{\mathrm{hg}} \times G\left(\mathbb{A}_{f}\right) / K \subset \operatorname{Sh}_{K}(G, X)(\mathbb{C})$.

Proposition 6.2.1. All $\ell$-Galois-generic points are Hodge-generic.

Proof. (See also [Pink 2005, Proposition 6.7].) Let $s \in \operatorname{Sh}(G, X)$ be $\ell$-Galois-generic and $(x, g) \in X \times G\left(\mathbb{A}_{f}\right)$ lifting $s_{\mathbb{C}} \in \operatorname{Sh}(G, X)(\mathbb{C})$. We may assume $g=1$ (see 
Section 4.4). Let $X_{x}$ denote the $\operatorname{MT}(x)(\mathbb{R})$-conjugacy class of $x: \mathbb{S} \rightarrow \operatorname{MT}(x)_{\mathbb{R}}$. The inclusion $\operatorname{MT}(x) \hookrightarrow G$ induces a morphism of Shimura data $\left(\operatorname{MT}(x), X_{x}\right) \rightarrow(G, X)$, and hence a morphism of Shimura varieties $\operatorname{Sh}\left(\operatorname{MT}(x), X_{x}\right) \rightarrow \operatorname{Sh}(G, X)$ and, for every neat compact open subgroup $K \subset G\left(\mathbb{A}_{f}\right)$, a morphism $\operatorname{Sh}_{K_{x}}\left(\operatorname{MT}(x), X_{x}\right) \rightarrow$ $\operatorname{Sh}_{K}(G, X)$, where we set $K_{x}:=K \cap \operatorname{MT}(x)\left(\mathbb{A}_{f}\right)$. Assume $K=\prod_{\ell} K_{\ell}$ and write $\Gamma:=K \cap G(\mathbb{Q})_{+}$. Let $s_{x} \in \operatorname{Sh}\left(\operatorname{MT}(x), X_{x}\right)$ be the point corresponding to the image of $x$ and let $E\left[K_{x}\right]$ denote the field of definition of the geometrically connected component $S\left[K_{x}, s_{x}\right]$ of $s_{x}\left[K_{x}\right]$ in $\operatorname{Sh}_{K_{x}}\left(\mathrm{MT}(x), X_{x}\right)$. The following diagram commutes (Section 4.1):

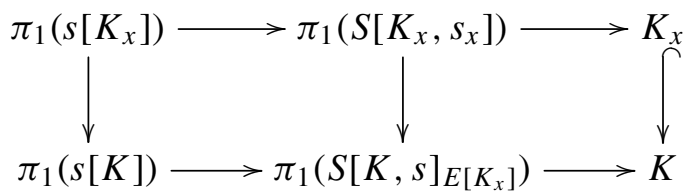

As $s \in \operatorname{Sh}(G, X)$ is $\ell$-Galois-generic and the image of

$$
\pi_{1}\left(s\left[K_{x}\right]\right) \rightarrow \pi_{1}(s[K]) \rightarrow \pi_{1}\left(S[K, s]_{E\left[K_{x}\right]}\right)
$$

is open in the image of $\pi_{1}(s[K]) \rightarrow \pi_{1}\left(S[K, s]_{E\left[K_{x}\right]}\right)$, the commutativity of the above diagram implies that $K[x, \ell] \subset \mathrm{MT}(x)\left(\mathbb{Q}_{\ell}\right)$ contains an open subgroup of $\Gamma_{\ell}^{-}$. As such a subgroup is Zariski-dense in $G_{\mathbb{Q}_{\ell}}^{\text {der }}$, this shows that $\operatorname{MT}(x)_{\mathbb{Q}_{\ell}}$ contains $G_{\mathbb{Q}_{\ell}}^{\text {der }}$, and hence that $\operatorname{MT}(x)$ contains $G^{\mathrm{der}}$. In particular, MT $(x)$ is normal in $G$. So every $G(\mathbb{R})$-conjugate of $x: \mathbb{S} \rightarrow \operatorname{MT}(x)_{\mathbb{R}}$ factors through $\operatorname{MT}(x)_{\mathbb{R}}$. Since $G$ is the generic Mumford-Tate group of $(G, X)$, this forces $\operatorname{MT}(x)=G$.

6.2.2. Pink [2005, Conjecture 6.8] conjectures that Hodge-generic points are Galoisgeneric. Thus, Theorem A reduces Pink's conjecture to proving that every Hodgegeneric point is $\ell$-Galois generic which, in the case of abelian schemes, is precisely the statement of the standard $(\ell$-adic) Mumford-Tate conjecture.

\subsection{Proof of Theorem A.}

6.3.1. Shimura data of Hodge type. Recall that a Shimura datum $\left(G_{2}, X_{2}\right)$ (as well as the associated Shimura variety) is said to be of Hodge type if there exists an embedding of Shimura data $f:\left(G_{2}, X_{2}\right) \hookrightarrow\left(G_{1}, X_{1}\right)$ with $\left(G_{1}, X_{1}\right)$ a Siegel Shimura datum. Let $K_{i} \subset G_{i}\left(\mathbb{A}_{f}\right)(i=1,2)$ be neat compact open subgroups such that $f\left(K_{2}\right) \subset K_{1}$. Let $s_{2} \in \operatorname{Sh}\left(G_{2}, X_{2}\right)$ and write $s_{1}:=f\left(s_{2}\right) \in \operatorname{Sh}\left(G_{1}, X_{1}\right)$. For simplicity, write $S_{i}:=S\left[K_{i}, s_{i}\right]$ for the geometrically connected component of $s_{i}\left[K_{i}\right]$ and let $E_{i}$ denote its field of definition for $i=1,2$. Let $A \rightarrow S_{1}$ denote the universal abelian scheme over $S_{1}$. Then the adelic representation

$$
\pi_{1}\left(S_{2}\right) \stackrel{f}{\rightarrow} \pi_{1}\left(S_{1 E_{2}}\right) \stackrel{\rho\left[K_{1}, S_{1}\right]}{\rightarrow} K_{1}
$$


coincides with the adelic representation attached to the abelian scheme $\left.A\right|_{S_{2}}:=$ $A \times_{S_{1 E_{2}}} S_{2} \rightarrow S_{2}$. But, as $f: K_{1} \hookrightarrow K_{2}$ is injective, one sees from Section 4.1 that

$$
\pi_{1}\left(S_{2}\right) \stackrel{f}{\rightarrow} \pi_{1}\left(S_{1 E_{2}}\right) \stackrel{\rho\left[K_{1}, S_{1}\right]}{\rightarrow} K_{1}
$$

and $\rho\left[K_{2}, s_{2}\right]: \pi_{1}\left(S_{2}\right) \rightarrow K_{2} \hookrightarrow K_{1}$ have the same Galois-generic and $\ell$-Galoisgeneric points. So, consider the following assertions for $s_{2}\left[K_{2}\right] \in S_{2}$ :

(1) Galois-generic with respect to $\rho\left[K_{2}, s_{2}\right]: \pi_{1}\left(S_{2}\right) \rightarrow K_{2} \subset G_{2}\left(\mathbb{A}_{f}\right)$;

(2) $\ell$-Galois-generic with respect to $\rho\left[K_{2}, s_{2}\right]: \pi_{1}\left(S_{2}\right) \rightarrow K_{2} \subset G_{2}\left(\mathbb{A}_{f}\right)$;

(3) Galois-generic with respect to the adelic representation attached to $\left.A\right|_{S_{2}} \rightarrow S_{2}$.

(4) $\ell$-Galois-generic with respect to the adelic representation attached to $\left.A\right|_{S_{2}} \rightarrow S_{2}$.

Then, from the above, (1) $\Leftrightarrow(3),(2) \Leftrightarrow$ (4) and from Theorem 3.3.2.2, (3) $\Leftrightarrow$ (4). This shows Theorem A for Shimura data of Hodge type.

6.3.2. Shimura data of abelian type. Recall that a Shimura datum $\left(G_{1}, X_{1}\right)$ (as well as the associated Shimura variety) is said to be of abelian type if there exists a Shimura datum $\left(G_{2}, X_{2}\right)$ of Hodge type and an isogeny $f: G_{2}^{\text {der }} \rightarrow G_{1}^{\text {der }}$ which induces an isomorphism of adjoint Shimura data $f:\left(G_{2}^{\mathrm{ad}}, X_{2}^{\mathrm{ad}}\right) \stackrel{2}{\rightarrow}\left(G_{1}^{\mathrm{ad}}, X_{1}^{\mathrm{ad}}\right)$. We refer to [Deligne 1971, §1.2, 1.3 and 2.7] and [Milne 2013, §10] for a detailed account of Shimura data of abelian type. These include essentially all Shimura data $(G, X)$ except those for which $G$ has simple factors of type $E_{6}, E_{7}$ and certain type $D$.

We can now conclude the proof of Theorem A. Start from an $\ell$-Galois-generic point $s_{1} \in \operatorname{Sh}\left(G_{1}, X_{1}\right)$. We may assume (Section 4.4) that the image $s_{1}^{\text {ad }}$ of $s_{1}$ in $\operatorname{Sh}\left(G_{1}^{\mathrm{ad}}, X_{1}^{\mathrm{ad}}\right) \simeq \operatorname{Sh}\left(G_{2}^{\mathrm{ad}}, X_{2}^{\mathrm{ad}}\right)$ lies in the image of $\operatorname{Sh}\left(G_{2}, X_{2}\right) \rightarrow \operatorname{Sh}\left(G_{2}^{\mathrm{ad}}, X_{2}^{\mathrm{ad}}\right)$. Fix $s_{2} \in \operatorname{Sh}\left(G_{2}, X_{2}\right)$ above $s_{1}^{\text {ad }}$. Because $s_{1} \in \operatorname{Sh}\left(G_{1}, X_{1}\right)$ is $\ell$-Galois-generic, $s_{1}^{\text {ad }} \in \operatorname{Sh}\left(G_{1}^{\text {ad }}, X_{1}^{\text {ad }}\right)$ is $\ell$-Galois-generic (by (1) $\Rightarrow(4)$ in Proposition 6.1.1) and $s_{2} \in \operatorname{Sh}\left(G_{2}, X_{2}\right)$ is $\ell$-Galois-generic (by (4) $\Rightarrow$ (1) in Proposition 6.1.1). As $\left(G_{2}, X_{2}\right)$ is of Hodge type, $s_{2} \in \operatorname{Sh}\left(G_{2}, X_{2}\right)$ is Galois-generic by Section 6.3.1. Thus $s_{1}^{\text {ad }} \in \operatorname{Sh}\left(G_{1}^{\text {ad }}, X_{1}^{\text {ad }}\right)$ is Galois-generic (by (1) $\Rightarrow(4)$ in Proposition 6.1.1) and $s_{1} \in \operatorname{Sh}\left(G_{1}, X_{1}\right)$ is Galois-generic (by (4) $\Rightarrow$ (1) in Proposition 6.1.1).

\section{Proof of Theorem B}

7.1. Generalized Hecke operators. Let $(G, X)$ be a Shimura datum. Let $X^{+} \subset X$ be a connected component and let $K \subset G\left(\mathbb{A}_{f}\right)$ be a neat compact open subgroup. Write $\Gamma:=G(\mathbb{Q})_{+} \cap K$. For every $a \in G\left(\mathbb{A}_{f}\right)$, let $T_{a}$ denote the Hecke operator $\cdot a^{-1}: \operatorname{Sh}(G, X)_{\mathbb{C}} \stackrel{\sim}{\rightarrow} \operatorname{Sh}(G, X)_{\mathbb{C}}$ and $T_{a, K}$ the corresponding algebraic correspondence

$$
\operatorname{Sh}_{K}(G, X)_{\mathbb{C}} \leftarrow \operatorname{Sh}(G, X)_{\mathbb{C}} \stackrel{a^{-1}}{\rightarrow} \operatorname{Sh}(G, X)_{\mathbb{C}} \rightarrow \operatorname{Sh}_{K}(G, X)_{\mathbb{C}}
$$


It is part of the definition of a canonical model that $T_{a, K}$ is defined over the reflex field $E:=E(G, X)$. For $a \in G(\mathbb{Q})_{+} K, T_{a, K}$ restricts to an algebraic correspondence $T_{a, \Gamma}$ (of degree 1 if $a \in K$ ) on $\operatorname{Sh}_{\Gamma}\left(G, X^{+}\right)_{\mathbb{C}}$, defined over $E_{K^{a^{-1}}}$ (see Section 4.2 for the notation):

$$
\operatorname{Sh}_{\Gamma}\left(G, X^{+}\right)_{\mathbb{C}} \leftarrow \operatorname{Sh}\left(G, X^{+}\right)_{\mathbb{C}} \stackrel{a}{\rightarrow} \operatorname{Sh}\left(G, X^{+}\right)_{\mathbb{C}} \rightarrow \operatorname{Sh}_{\Gamma}\left(G, X^{+}\right)_{\mathbb{C}}
$$

Here, we write

$$
\operatorname{Sh}\left(G, X^{+}\right)_{\mathbb{C}}:=\lim _{\Gamma} \operatorname{Sh}_{\Gamma}\left(G, X^{+}\right)_{\mathbb{C}}
$$

where the projective limit is taken over all congruence subgroups $\Gamma \subset G(\mathbb{Q})$. Recall that $\operatorname{Sh}\left(G, X^{+}\right)_{\mathbb{C}}$ identifies with the connected component of $\operatorname{Sh}(G, X)$ containing the image of $X^{+} \times\{1\}$ [Deligne 1971, §1.8]. For $s=\Gamma x \in \operatorname{Sh}_{\Gamma}\left(G, X^{+}\right)(\mathbb{C})$ we set

$$
T_{a, \Gamma}(s)=\{\Gamma a \gamma x \mid \gamma \in \Gamma\} .
$$

More generally, for a subset $A \subset G(\mathbb{Q})_{+}$, we set

$$
T_{A, \Gamma}(s):=\bigcup_{a \in A} T_{a, \Gamma}(s) \subset \operatorname{Sh}_{\Gamma}\left(G, X^{+}\right)(\mathbb{C})
$$

for its $A$-Hecke orbit. For $A=G(\mathbb{Q})_{+}$we simply write $T_{A, \Gamma}(s)=: T_{\Gamma}(s)$ for the full Hecke orbit of $s$.

Let $\operatorname{Aut}\left(G, X^{+}\right)$denote the group automorphisms of $G$ defined over $\mathbb{Q}$ and stabilizing $X^{+}$. For every $\phi \in \operatorname{Aut}\left(G, X^{+}\right)$, the corresponding generalized Hecke operator $T_{\phi}$ is the algebraic correspondence

$$
\operatorname{Sh}_{\Gamma}\left(G, X^{+}\right)_{\mathbb{C}} \leftarrow \operatorname{Sh}\left(G, X^{+}\right)_{\mathbb{C}} \stackrel{\phi .}{\rightarrow} \operatorname{Sh}\left(G, X^{+}\right)_{\mathbb{C}} \rightarrow \operatorname{Sh}_{\Gamma}\left(G, X^{+}\right)_{\mathbb{C}}
$$

For $s=\Gamma x \in \operatorname{Sh}_{\Gamma}\left(G, X^{+}\right)(\mathbb{C})$ we set

$$
T_{\phi, \Gamma}(s)=\{\Gamma \phi(\gamma) \phi(x) \mid \gamma \in \Gamma\} .
$$

More generally, for a subset $\Phi \subset \operatorname{Aut}\left(G, X^{+}\right)$, we set

$$
T_{\Phi, \Gamma}(s):=\bigcup_{\phi \in \Phi} T_{\phi, \Gamma}(s) \subset \operatorname{Sh}_{\Gamma}\left(G, X^{+}\right)(\mathbb{C})
$$

for its $\Phi$-Hecke orbit. The usual full Hecke orbit $T_{\Gamma}(s)$ coincides with the $\Phi$ Hecke orbit $T_{\Phi, \Gamma}(s)$ for $\Phi$ the image of $G(\mathbb{Q})_{+} \rightarrow \operatorname{Aut}\left(G, X^{+}\right)$given by inner automorphisms. For $\Phi=\operatorname{Aut}\left(G, X^{+}\right)$we simply write $T_{\Phi, \Gamma}(s)=: \widehat{T}_{\Gamma}(s)$ for the full generalized Hecke orbit of $s$.

If $\Gamma$ is obvious from the context, we will omit it from the notation. The above definitions of Hecke orbits extend as they are to arithmetic subgroups $\Gamma \subset G(\mathbb{Q})_{+}$.

For the comparison between usual Hecke orbits and generalized Hecke orbits, see [Orr 2013, §4.1.1]; let us only point out the following observation, which will be used in the proof of Theorem B. 
Assume $G$ is adjoint. For an arithmetic subgroup $\Gamma \subset G(\mathbb{Q})^{+}$, write again $\operatorname{Sh}_{\Gamma}\left(G, X^{+}\right)_{\mathbb{C}}$ for the complex algebraic variety underlying $\Gamma \backslash X^{+}$[Baily and Borel 1966].

Lemma 7.1.1. For every congruence (resp. arithmetic) subgroup $\Gamma \subset G(\mathbb{Q})^{+}$there exists a congruence (resp. an arithmetic) subgroup $\Gamma^{\prime} \subset \Gamma$ such that, for every subset $\Phi \subset \operatorname{Aut}\left(G, X^{+}\right)$and $s \in \operatorname{Sh}_{\Gamma}\left(G, X^{+}\right)$, the inverse image of $T_{\Phi, \Gamma}(s)$ by $p_{\Gamma^{\prime}, \Gamma}: \operatorname{Sh}_{\Gamma^{\prime}}\left(G, X^{+}\right) \rightarrow \operatorname{Sh}_{\Gamma}\left(G, X^{+}\right)$is contained in a finite union of usual Hecke orbits on $\operatorname{Sh}_{\Gamma^{\prime}}\left(G, X^{+}\right)$.

Proof. As $G$ is adjoint, the quotient $\operatorname{Aut}\left(G, X^{+}\right) / G(\mathbb{Q})^{+}$is finite. Choose a system of representatives $\phi_{1}, \ldots, \phi_{r}$ for $\operatorname{Aut}\left(G, X^{+}\right) / G(\mathbb{Q})^{+}$and set

$$
\Gamma^{\prime}:=\bigcap_{1 \leq i \leq r} \phi_{i}(\Gamma) \subset \Gamma, \quad \Gamma^{\prime \prime}:=\bigcap_{1 \leq i \leq r} \phi_{i}\left(\Gamma^{\prime}\right) \subset \Gamma^{\prime} .
$$

Note that, by construction, if $\Gamma$ is a congruence (resp. an arithmetic) subgroup then $\Gamma^{\prime}$ and $\Gamma^{\prime \prime}$ are again congruence (resp. arithmetic) subgroups. Fix systems of representatives

- $\gamma_{j}, j=1, \ldots, s$, of $\Gamma / \Gamma^{\prime}$

- $\alpha_{k}, k=1, \ldots, t$, of $\Gamma / \Gamma^{\prime \prime}$;

- and $\alpha_{i, l}, l=1, \ldots, t_{i}$, of $\phi_{i}\left(\Gamma^{\prime}\right) / \Gamma^{\prime \prime}$ for $i=1, \ldots, r$.

For an arbitrary element $\phi=a-a^{-1} \circ \phi_{i} \in \operatorname{Aut}\left(G, X^{+}\right)$, we can then compute explicitly

$$
p_{\Gamma^{\prime \prime}, \Gamma}^{-1}\left(T_{\phi, \Gamma}(s)\right)=\bigcup_{1 \leq j \leq s} \bigcup_{1 \leq k \leq t} \bigcup_{1 \leq l \leq t_{i}} T_{\alpha_{k} a \alpha_{l, i}, \Gamma^{\prime \prime}}\left(\phi_{i}\left(\gamma_{j} x\right)\right) .
$$

This shows that for every subset $\Phi \subset \operatorname{Aut}\left(G, X^{+}\right)$and point $s \in \operatorname{Sh}_{\Gamma}\left(G, X^{+}\right)$, we have that $p_{\Gamma^{\prime \prime}, \Gamma}^{-1}\left(T_{\Phi, \Gamma}(s)\right)$ is contained in a finite union of usual Hecke orbits on $\operatorname{Sh}_{\Gamma^{\prime \prime}}\left(G, X^{+}\right)$.

7.2. Equidistribution. Let $G$ be a connected $\mathbb{Q}$-simple algebraic group of noncompact type and let $G(\mathbb{R})^{+} \subset G(\mathbb{R})$ denote the connected component of 1 in $G(\mathbb{R})$. Set $G(\mathbb{Q})^{+}:=G(\mathbb{Q}) \cap G(\mathbb{R})^{+}$. Fix an arithmetic subgroup $\Gamma \subset G(\mathbb{Q})^{+}$. Then $\Gamma$ is a lattice in $G(\mathbb{R})^{+}$[Borel and Harish-Chandra 1962]; let $\mu$ denote the normalized Haar measure on $\Gamma \backslash G(\mathbb{R})^{+}$. For a function $f: \Gamma \backslash G(\mathbb{R})^{+} \rightarrow \mathbb{C}$ and an element $a \in G(\mathbb{Q})^{+}$, define its Hecke transform $T_{a}(f): \Gamma \backslash G(\mathbb{R})^{+} \rightarrow \mathbb{C}$ by

$$
T_{a}(f)(y)=\frac{1}{\operatorname{deg}_{\Gamma}(a)} \sum_{y^{\prime} \in T_{a}(y)} f\left(y^{\prime}\right),
$$

where $\operatorname{deg}_{\Gamma}(a)=|\Gamma a \Gamma / \Gamma|=\left[\Gamma: \Gamma \cap a \Gamma a^{-1}\right]$. 
Fact 7.2.1 ([Eskin and Oh 2006, Theorem 1.2]; see also [Burger and Sarnak 1991, “Theorem 5.2"]). For every sequence $\underline{a}=\left(a_{n}\right)$ of elements in $G(\mathbb{Q})^{+}$with

$$
\lim _{n \rightarrow+\infty} \operatorname{deg}_{\Gamma}\left(a_{n}\right)=+\infty
$$

and for every continuous bounded function $f: \Gamma \backslash G(\mathbb{R})^{+} \rightarrow \mathbb{C}$ and $y \in \Gamma \backslash G(\mathbb{R})^{+}$, we have

$$
\lim _{n \rightarrow+\infty} T_{a_{n}}(f)(y)=\int_{\Gamma \backslash G(\mathbb{R})^{+}} f d \mu .
$$

In particular, for every $y \in \Gamma \backslash G(\mathbb{R})^{+}$the set

$$
T_{\underline{a}}(y):=\bigcup_{n \geq 1} T_{a_{n}}(y)
$$

is dense in $\Gamma \backslash G(\mathbb{R})^{+}$. To exploit Fact 7.2.1, we need the following general finiteness result about Hecke operators of bounded degree.

Theorem 7.2.2. Let $G$ be a connected semisimple group over $\mathbb{Q}$ of noncompact type $^{2}$ and let $\Gamma \subset G(\mathbb{Q})$ be an arithmetic subgroup. Then for every integer $d \geq 1$ there are only finitely many double-classes $\Gamma a \Gamma \in \Gamma \backslash G(\mathbb{Q}) / \Gamma$ with $\operatorname{deg}_{\Gamma}(a) \leq d$.

Theorem 7.2.2 will be proved in Section 8 .

Let $(G, X)$ be a Shimura datum. Fix a connected component $X^{+} \subset X$ and a neat arithmetic subgroup $\Gamma \subset G^{\text {der }}(\mathbb{Q})_{+}$.

Corollary 7.2.3 (compare with [Pink 2005, Theorem 7.5]). Assume $G$ is almost $\mathbb{Q}$-simple. Then for every $s_{\Gamma} \in \operatorname{Sh}_{\Gamma}\left(G, X^{+}\right)$and for every sequence $\phi=\left(\phi_{n}\right)$ in $\operatorname{Aut}\left(G, X^{+}\right)$, the set $T_{\phi, \Gamma}\left(s_{\Gamma}\right)$ is either finite or Zariski-dense in $\operatorname{Sh}_{\Gamma}\left(G, X^{+}\right)$.

Proof. Let $X^{\text {ad }}$ denote the $G^{\text {ad }}(\mathbb{R})$-conjugacy class of $p^{\text {ad }} \circ x: \mathbb{S} \rightarrow G_{\mathbb{R}}^{\text {ad }}$ for one (equivalently every) $x \in X$. Then $p^{\text {ad }}: X \hookrightarrow X^{\text {ad }}$ identifies $X$ with a union of connected components of $X^{\text {ad }}$. As $\Gamma \subset G^{\text {der }}(\mathbb{Q})_{+}, p^{\text {ad }}: G \rightarrow G^{\text {ad }}$ maps $\Gamma$ bijectively onto its image $\Gamma^{\mathrm{ad}}:=p^{\mathrm{ad}}(\Gamma)$. Thus the morphism of Shimura data $p^{\text {ad }}:(G, X) \rightarrow\left(G^{\mathrm{ad}}, X^{\mathrm{ad}}\right)$ induces an isomorphism of schemes over $\mathbb{C}$

$$
p^{\mathrm{ad}}: \operatorname{Sh}_{\Gamma}\left(G, X^{+}\right) \stackrel{\sim}{\rightarrow} \operatorname{Sh}_{\Gamma^{\mathrm{ad}}}\left(G^{\mathrm{ad}}, X^{+}\right),
$$

and this isomorphism maps $T_{\underline{\phi}, \Gamma}\left(s_{\Gamma}\right)$ bijectively onto $T_{p^{\text {ad }}(\underline{\phi}), \Gamma \text { ad }}\left(p^{\text {ad }}\left(s_{\Gamma}\right)\right)$, where we write again

$$
p^{\text {ad }}: \operatorname{Aut}\left(G, X^{+}\right) \rightarrow \operatorname{Aut}\left(G^{\text {ad }}, X^{+}\right)
$$

for the morphism of groups induced by $p^{\text {ad }}: G \rightarrow G^{\text {ad }}$. Thus, we may assume $G$ is adjoint. Next, for every arithmetic subgroup $\Gamma^{\prime} \subset \Gamma$, the quotient map

${ }^{2}$ If we remove the assumption that $G$ is of noncompact type, Theorem 7.2.2 becomes trivially false. Indeed, if $G$ is $\mathbb{Q}$-simple of compact type then $\Gamma$ is always finite while $G(\mathbb{Q})$ is always infinite. 
$p_{\Gamma^{\prime}, \Gamma}: \operatorname{Sh}_{\Gamma^{\prime}}\left(G, X^{+}\right) \rightarrow \operatorname{Sh}_{\Gamma}\left(G, X^{+}\right)$is a finite cover so it is enough to prove that $p_{\Gamma^{\prime}, \Gamma}^{-1}\left(T_{\phi, \Gamma}\left(s_{\Gamma}\right)\right)$ is finite. In particular, by Lemma 7.1.1 we may assume that $\phi_{n}=a_{n} \in G(\mathbb{Q})_{+}, n \geq 0$. Then, assume that the Zariski-closure $Z$ of $T_{\underline{a}, \Gamma}\left(s_{\Gamma}\right)$ in $\operatorname{Sh}_{\Gamma}\left(G, X^{+}\right)$is a strict closed subscheme. So $Z(\mathbb{C}) \subsetneq \operatorname{Sh}_{\Gamma}\left(G, X^{+}\right)(\mathbb{C})=\Gamma \backslash X^{+}$is a strict closed analytic subset. As the canonical map $p_{\Gamma}: \Gamma \backslash G(\mathbb{R})^{+} \rightarrow \Gamma \backslash X^{+}$is analytic and surjective, $p_{\Gamma}^{-1}(Z(\mathbb{C})) \subsetneq \Gamma \backslash G(\mathbb{R})^{+}$is again a strict closed analytic subset. As $p_{\Gamma}^{-1}\left(T_{\underline{a}, \Gamma}\left(s_{\Gamma}\right)\right)=T_{\underline{a}}(1) \subset p_{\Gamma}^{-1}(Z(\mathbb{C}))$, we have

$$
T_{\underline{a}}(1)^{-} \subset p_{\Gamma}^{-1}(Z(\mathbb{C}))^{-}=p_{\Gamma}^{-1}(Z(\mathbb{C})) \subsetneq \Gamma \backslash G(\mathbb{R})^{+} .
$$

Then by Fact 7.2.1, $\operatorname{deg}_{\Gamma}\left(a_{n}\right)$ is bounded which, in turn, implies by Theorem 7.2.2 that the set

$$
\left\{\Gamma a_{n} \Gamma \mid n \geq 0\right\} \subset \Gamma \backslash G(\mathbb{Q}) / \Gamma
$$

is finite. Hence $T_{\underline{a}, \Gamma}\left(s_{\Gamma}\right)=p_{\Gamma}\left(T_{\underline{a}}(1)\right)$ is finite as well.

7.3. Proof of Theorem B. (Compare with [Pink 2005, proof of Theorem 7.6]). Fix a Galois-generic point $s \in \operatorname{Sh}(G, X)$ such that $s_{\mathbb{C}}=G(\mathbb{Q})(x, 1)$, and let $X^{+} \subset X$ denote the connected component of $x$. Setting $\Gamma_{0}:=K_{0} \cap G(\mathbb{Q})_{+}$, we then have $S\left[K_{0}, s\right]=\operatorname{Sh}_{\Gamma_{0}}\left(G, X^{+}\right)$. Recall from Proposition 6.1.1 that $s_{E^{\text {ab }}} \in$ $\operatorname{Sh}(G, X)_{E^{\text {ab }}}$ is again Galois-generic. So, to prove Theorem B, we may and will work over $E^{\mathrm{ab}}$ (without mentioning it explicitly in the notation, for simplicity). Then $\rho\left[K_{0}\right]: \pi_{1}\left(\operatorname{Sh}_{\Gamma_{0}}\left(G, X^{+}\right)\right) \rightarrow \Gamma_{0}^{-}$is completely determined by the tower of connected étale covers

$$
p_{\Gamma, \Gamma_{0}}: \operatorname{Sh}_{\Gamma}\left(G, X^{+}\right) \rightarrow \operatorname{Sh}_{\Gamma_{0}}\left(G, X^{+}\right) .
$$

Let $Z \hookrightarrow \operatorname{Sh}_{\Gamma}\left(G, X^{+}\right)_{\mathbb{C}}$ be a closed subvariety containing an infinite subset $T$ of $\widehat{T}_{\Gamma}(s)$.

- Reduction to the case where $s\left[K_{0}\right] \in \operatorname{Sh}_{\Gamma_{0}}\left(G, X^{+}\right)$is strictly Galois-generic and $Z$ is defined over the residue field $k\left(s\left[K_{0}\right]\right)$ of $s\left[K_{0}\right]$. As all the points in $\widehat{T}_{\Gamma_{0}}(s)$ are defined over the algebraic closure of $k\left(s\left[K_{0}\right]\right)$, up to replacing $Z$ by the irreducible components of the Zariski closure of $T$ in $\operatorname{Sh}_{\Gamma_{0}}\left(G, X^{+}\right)$, we may assume $Z$ is defined over a finite field extension $F$ of $k\left(s\left[K_{0}\right]\right)$. As $s\left[K_{0}\right]_{F} \in \mathrm{Sh}_{\Gamma_{0}}\left(G, X^{+}\right)_{F}$ is again Galois-generic, up to replacing $s\left[K_{0}\right]$ by $s\left[K_{0}\right]_{F}$, we may assume $F=k\left(s\left[K_{0}\right]\right)$. Then we can find a congruence subgroup $\Gamma:=K \cap G\left(\mathbb{A}_{f}\right) \subset \Gamma_{0}$ with $\Gamma^{-} \subset \Pi_{s\left[K_{0}\right]}$. Hence $s\left[K_{0}\right]$ lifts to a strictly Galois-generic point $s[K] \in \operatorname{Sh}_{\Gamma}\left(G, X^{+}\right)_{F}$; see 3.2.6. Then $p_{\Gamma, \Gamma_{0}}^{-1} Z \hookrightarrow \operatorname{Sh}_{\Gamma}\left(G, X^{+}\right)_{F}$ is again a closed subvariety which contains an infinite subset of $\widehat{T}_{\Gamma}(s[K])$ (just observe that, for every $\phi \in \operatorname{Aut}\left(G, X^{+}\right)$,

$$
p_{\Gamma, \Gamma_{0}}^{-1}\left(T_{\phi, \Gamma_{0}}\left(s\left[K_{0}\right]\right)\right)=\bigcup_{\gamma_{1}, \gamma_{2} \in \Gamma_{0} / \Gamma} T_{\phi_{\gamma_{1}, \gamma_{2}}, \Gamma}\left(s[K]_{F}\right),
$$


where $\phi_{\gamma_{1}, \gamma_{2}} \in \operatorname{Aut}\left(G, X^{+}\right)$is defined by $\phi_{\gamma_{1}, \gamma_{2}}(g)=\gamma_{1} \phi\left(\gamma_{2}\right) \phi(g)\left(\gamma_{1} \phi\left(\gamma_{2}\right)\right)^{-1}$, $g \in G)$. As it is enough to show that $p_{\Gamma, \Gamma_{0}}^{-1} Z=\operatorname{Sh}_{\Gamma}\left(G, X^{+}\right)_{F}$, up to replacing $E^{\text {ab }}$ with $F, \operatorname{Sh}_{\Gamma_{0}}\left(G, X^{+}\right)$with $\operatorname{Sh}_{\Gamma}\left(G, X^{+}\right)_{F}, s\left[K_{0}\right]$ with $s[K]$ and $Z$ with $p_{\Gamma, \Gamma_{0}}^{-1} Z$, without loss of generality we may assume that $s\left[K_{0}\right] \in \operatorname{Sh}_{\Gamma_{0}}\left(G, X^{+}\right)$is strictly Galois-generic and that $Z$ is defined over $F=k\left(s\left[K_{0}\right]\right)$.

- Conclusion. As $s\left[K_{0}\right]$ is strictly Galois-generic, for every generalized Hecke operator $\phi \in \operatorname{Aut}\left(G, X^{+}\right)$, the group $\pi_{1}\left(k\left(s\left[K_{0}\right]\right)\right)$ acts transitively on $T_{\phi, \Gamma_{0}}\left(s\left[K_{0}\right]\right) \subset$ $\operatorname{Sh}_{\Gamma_{0}}\left(G, X^{+}\right)$. In particular, for each $\phi \in \operatorname{Aut}\left(G, X^{+}\right)$such that $Z \cap T_{\phi, \Gamma_{0}}\left(s\left[K_{0}\right]\right) \neq \varnothing$ we have $T_{\phi, \Gamma_{0}}\left(s\left[K_{0}\right]\right) \subset Z$ (recall that we assume $Z$ is defined over $k\left(s\left[K_{0}\right]\right)$ ). Now, consider a sequence $\phi=\left(\phi_{n}\right)$ of elements in $\operatorname{Aut}\left(G, X^{+}\right)$such that $Z$ contains a point $s_{n} \in T_{\phi_{n}, \Gamma_{0}}\left(s\left[\overline{\left.K_{0}\right]}\right), n \geq 1\right.$ with $s_{n} \neq s_{m}$ for $n \neq m$. Then

$$
T_{\underline{\phi}, \Gamma_{0}}\left(s\left[K_{0}\right]\right)=\bigcup_{n \geq 1} T_{\phi_{n}, \Gamma_{0}}\left(s\left[K_{0}\right]\right) \subset Z
$$

is infinite by construction. So, the conclusion follows from Corollary 7.2.3.

\section{Degree of Hecke operators}

8.1. Formal lemmas. Let $G$ be a group. For every subgroup $K, K^{\prime} \subset G$ such that $K \equiv K^{\prime}$, set

$$
\left[K: K^{\prime}\right]:=\frac{\left[K: K \cap K^{\prime}\right]}{\left[K^{\prime}: K \cap K^{\prime}\right]} \in \mathbb{Q} .
$$

For a subgroup $K \subset G$, let $K \equiv$ be the set of all $g \in G$ such that $K$ and $g K^{-1}$ are commensurable. Then $K^{\equiv_{G}} \subset G$ is a subgroup containing $K$ and for every subgroup $K^{\prime} \subset G$ such that $K^{\prime} \equiv K$, we have $K^{\equiv}=K^{\prime} \equiv$. For $g \in K^{\equiv}$, define the degree of $g$ with respect to $K$ as

$$
\operatorname{deg}_{K}(g)=\left[K: K \cap g K g^{-1}\right]=|K \backslash K g K| .
$$

For subgroups $K \subset U \subset G$, let $\operatorname{Cor}_{U}(K):=\bigcap_{u \in U} u K u^{-1} \subset K$ denote the largest subgroup of $K$ which is normalized by $U$. Equivalently, $\operatorname{Cor}_{U}(K)$ is the kernel of $U$ acting on $U / K$ by left translation. In particular, if $[U: K]$ is finite then $\left[U: \operatorname{Cor}_{U}(K)\right]$ is also finite and $\left[U: \operatorname{Cor}_{U}(K)\right] \leq[U: K]$ !.

Lemma 8.1.1. For subgroups $K, K^{\prime} \subset G$ such that $K \equiv K^{\prime}$ and $a \in K^{\equiv}$, we have $\operatorname{deg}_{K}(a) \leq C_{K, K^{\prime}} \operatorname{deg}_{K^{\prime}}(a)$, where $C_{K, K^{\prime}}=\left[K: \operatorname{Cor}_{K}\left(K \cap K^{\prime}\right)\right]\left[K^{\prime}: \operatorname{Cor}_{K}\left(K \cap K^{\prime}\right)\right]$.

Proof. Observe first that

(i) if $K^{\prime} \subset K$ then $\operatorname{deg}_{K^{\prime}}(a) \leq\left[K: K^{\prime}\right] \operatorname{deg}_{K}(a)$, and

(ii) if furthermore $K^{\prime} \subset K$ is normal, then $\operatorname{deg}_{K}(a) \leq\left[K: K^{\prime}\right] \operatorname{deg}_{K^{\prime}}(a)$. 
The proof of (i) is straightforward. As for the proof of (ii), if $K^{\prime} \subset K$ is normal then

$$
\operatorname{deg}_{K^{\prime}}(k a)=\left|K^{\prime} / K^{\prime} \cap k a K^{\prime}(k a)^{-1}\right|=\left|K^{\prime} / k^{-1} K^{\prime} k \cap a K^{\prime} a^{-1}\right|=\operatorname{deg}_{K^{\prime}}(a)
$$

and similarly, $\operatorname{deg}_{K^{\prime}}(a k)=\operatorname{deg}_{K^{\prime}}(a)$. Let $R \subset K$ be a set of representatives for the left cosets of $K^{\prime}$ in $K$. By normality, $R$ is also a set of representatives for the right cosets of $K^{\prime}$ in $K$. Hence,

$$
\begin{aligned}
{\left[K: K^{\prime}\right] \operatorname{deg}_{K}(a) } & =\left|K^{\prime} \backslash K\right||K \backslash K a K|=\left|K^{\prime} \backslash K a K\right| \\
& \leq \sum_{x, y \in R} \mid K^{\prime} \backslash K^{\prime} \text { xay } K^{\prime} \mid=\sum_{x, y \in R} \operatorname{deg}(x a y)=\left[K: K^{\prime}\right]^{2} \operatorname{deg}_{K^{\prime}}(a),
\end{aligned}
$$

where the last equality follows from $(*)$. The assertion in Lemma 8.1.1 now follows from the combination of (i) and (ii):

$$
\begin{array}{rlr}
\operatorname{deg}_{K}(a) & \leq\left[K: \operatorname{Cor}_{K}\left(K \cap K^{\prime}\right)\right] \operatorname{deg}_{\operatorname{Cor}_{K}\left(K \cap K^{\prime}\right)}(a) & \text { (by (i)) } \\
& \leq\left[K: \operatorname{Cor}_{K}\left(K \cap K^{\prime}\right)\right]\left[K^{\prime}: \operatorname{Cor}_{K}\left(K \cap K^{\prime}\right)\right] \operatorname{deg}_{K^{\prime}}(a) & \text { (by (ii)). }
\end{array}
$$

Definition 8.1.2. For a subgroup $K \subset G$, we say that property $\star(G, K)$ holds if for every integer $d \geq 1$ there are only finitely many $K$-double classes $K a K \in K \backslash G / K$ with $\operatorname{deg}_{K}(a) \leq d$.

Lemma 8.1.3. Let $\varphi: G^{\prime} \rightarrow G$ be a morphism of groups and $K^{\prime} \subset G^{\prime}, K \subset G$ two subgroups. Assume that $\operatorname{ker}(\varphi),\left[G: \varphi\left(G^{\prime}\right)\right]$ are finite and $K \equiv \varphi\left(K^{\prime}\right) \subset G$. Then $\star(G, K)$ holds if and only if $\star\left(G^{\prime}, K^{\prime}\right)$ holds.

Proof. First, consider the case where $G^{\prime}=G$ and $\varphi$ is the identity. As the situation is symmetric in $K, K^{\prime}$, it is enough to show the implication $\star(G, K) \Rightarrow \star\left(G^{\prime}, K^{\prime}\right)$. Set $K^{\prime \prime}:=K \cap K^{\prime}$ and fix an integer $d \geq 1$. Let $a \in K^{\equiv}\left(=K^{\prime \equiv}\right)$ with $\operatorname{deg}_{K^{\prime}}(a) \leq d$. From Lemma 8.1.1(ii), $\operatorname{deg}_{K}(a) \leq C_{K^{\prime}, K} d$. From $\star(G, K)$, there are only finitely many possibilities for the $K$-double class $K a K \in K \backslash G / K$. But, then there are also only finitely many possibilities for the $K^{\prime}$-double class $K^{\prime} a K^{\prime} \in K^{\prime} \backslash G / K^{\prime}$ since the induced maps $K^{\prime \prime} \backslash G / K^{\prime \prime} \rightarrow K \backslash G / K$ and $K^{\prime \prime} \backslash G / K^{\prime \prime} \rightarrow K^{\prime} \backslash G / K^{\prime}$ are both surjective with finite fibers.

In particular, $\star(G, K)$ holds if and only if $\star\left(G, \varphi\left(K^{\prime}\right)\right)$ holds. So, in the following, we may and will assume that $K=\varphi\left(K^{\prime}\right)$.

Then the assumption that $\operatorname{ker}(\varphi)$ is finite ensures that the induced map

$$
K^{\prime} \backslash G^{\prime} / K^{\prime} \rightarrow K \backslash G / K
$$

has finite fibers. The implication $\star(G, K) \Rightarrow \star\left(G^{\prime}, K^{\prime}\right)$ then follows from the inequality $\operatorname{deg}_{K}\left(\varphi\left(a^{\prime}\right)\right) \leq \operatorname{deg}_{K^{\prime}}\left(a^{\prime}\right), a^{\prime} \in G^{\prime}$. 
For the implication $\star\left(G^{\prime}, K^{\prime}\right) \Rightarrow \star(G, K)$, observe that

$$
\operatorname{deg}_{K}(a b) \leq \operatorname{deg}_{K}(a) \operatorname{deg}_{K}(b), \quad a, b \in G
$$

(just note that $K a b K \subset K a K b K$ ). Let $\Delta$ denote a (finite) set of representatives of left cosets of $\varphi\left(G^{\prime}\right)$ in $G$. Then for every $a \in G$ there exists (a unique) $\delta_{a} \in \Delta$ such that $a \delta_{a}^{-1}=\varphi\left(a^{\prime}\right)$ for some $a^{\prime} \in G^{\prime}$. In particular,

$$
\operatorname{deg}_{K^{\prime}}\left(a^{\prime}\right) \leq \operatorname{deg}_{K}\left(\varphi\left(a^{\prime}\right)\right) \leq \min \left\{\operatorname{deg}_{K}\left(\delta^{-1}\right) \mid \delta \in \Delta\right\} \operatorname{deg}_{K}(a) .
$$

As a result, to prove Theorem 7.2.2, we may assume that $\Gamma \subset G(\mathbb{Q})$ is a congruence subgroup. So, let $K \subset G\left(\mathbb{A}_{f}\right)$ be a compact open subgroup such that $\Gamma=K \cap G(\mathbb{Q})$. By shrinking $K$, we may assume $K$ is of the form

$$
K=K_{\mathcal{P}} K^{\mathcal{P}},
$$

where $\mathcal{P}$ is a finite set of primes containing the primes where $G$ ramifies, where $K_{\mathcal{P}}=\prod_{p \in \mathcal{P}} K_{p}$ with $K_{p} \subset G\left(\mathbb{Q}_{p}\right)$ compact open and where $K^{\mathcal{P}}=\prod_{p \notin \mathcal{P}} K_{p}$ with $K_{p} \subset G\left(\mathbb{Q}_{p}\right)$ hyperspecial.

8.2. Degree of adelic Hecke operators. In this section, we reduce the adelic variant of Theorem 7.2.2, Theorem 8.2.1, to statements (Lemmas 8.2.2, 8.2.5) about the degree of local ( $p$-adic) Hecke operators.

Theorem 8.2.1. Let $G$ be a connected semisimple group over $\mathbb{Q}$. Then $\star\left(G\left(\mathbb{A}_{f}\right), K\right)$ holds for every compact open subgroup $K \subset G\left(\mathbb{A}_{f}\right)$.

Lemma 8.2.2. Let $G$ be a connected semisimple group over $\mathbb{Q}_{p}$. Then $\star\left(G\left(\mathbb{Q}_{p}\right), K\right)$ holds for every compact open subgroup $K \subset G\left(\mathbb{Q}_{p}\right)$.

Remark 8.2.3. More precisely, let $n_{d}\left(G\left(\mathbb{Q}_{p}\right), K\right)$ denote the number of double classes $K a K$ for $a \in G\left(\mathbb{Q}_{p}\right)$ with $\operatorname{deg}_{K}(a) \leq d$, and let $B \subset \mathbb{G}\left(\mathbb{Q}_{p}\right)$ be an Iwahori subgroup. Then

$$
n_{d}\left(G\left(\mathbb{Q}_{p}\right), K\right) \leq\left|\left\{w \in W_{G} \mid \ell(w) \leq \frac{\ln \left(C_{B, K} d\right)}{\ln (p)}\right\}\right|,
$$

where $W_{G}$ denotes the affine Weil group of $G, \ell: W_{G} \rightarrow \mathbb{Z}_{\geq 0}$ the length function on it and $C_{B, K}$ is the constant of Lemma 8.1.1.

Lemma 8.2.4 (definition of $\iota(-)$ ). Let $G^{\prime} \rightarrow G$ be an isogeny of algebraic groups over $\mathbb{Q}_{p}$. Assume that the degree $N$ of its kernel $\mu$ is at most $p$. Then there exists a constant $\iota(N)$ depending only on $N$ (but not on $p$ ) such that

$$
\left|\operatorname{coker}\left(G^{\prime}\left(\mathbb{Q}_{p}\right) \rightarrow G\left(\mathbb{Q}_{p}\right)\right)\right| \leq \iota(N) .
$$

Proof. By the long exact sequence in Galois cohomology, it is enough to show that there exists a constant $\iota(N)$ depending only on $N$ such that $\left|\mathrm{H}^{1}\left(\mathbb{Q}_{p}, \mu\left(\overline{\mathbb{Q}}_{p}\right)\right)\right| \leq \iota(N)$. For this, let $E / \mathbb{Q}_{p}$ denote the finite Galois extension corresponding to the kernel of 
the action of $\pi_{1}\left(\mathbb{Q}_{p}\right)$ on $\mu\left(\overline{\mathbb{Q}}_{p}\right)$. We have $\left[E: \mathbb{Q}_{p}\right] \leq N$ !. As $p$ is prime to $N$ !, the number of extensions of degree $\leq N ! N$ of $\mathbb{Q}_{p}$ is

$$
c(N):=\sum_{n=1}^{N ! N} \sum_{d \mid n} \frac{n}{d} .
$$

Let $E_{N} / E$ denote the finite Galois extension corresponding to the open subgroup $\bigcap_{E^{\prime}} \pi_{1}\left(E^{\prime}\right) \subset \pi_{1}(E)$, where $E^{\prime} / E$ describes all Galois extensions of degree $\leq N$ in $\overline{\mathbb{Q}}_{p} / E$. We have $\left[E_{N}: \mathbb{Q}_{p}\right] \leq N ! c(N) N$. By construction, the restriction map $\mathrm{H}^{1}\left(\mathbb{Q}_{p}, \mu\left(\overline{\mathbb{Q}}_{p}\right)\right) \rightarrow \mathrm{H}^{1}\left(E_{N}, \mu\left(\overline{\mathbb{Q}}_{p}\right)\right)$ is trivial. So, by the inflation-restriction exact sequence, we get an isomorphism $\mathrm{H}^{1}\left(E_{N} \mid \mathbb{Q}_{p}, \mu\left(\overline{\mathbb{Q}}_{p}\right)\right) \stackrel{\sim}{\rightarrow} \mathrm{H}^{1}\left(\mathbb{Q}_{p}, \mu\left(\overline{\mathbb{Q}}_{p}\right)\right)$ and we can take $\iota(N):=N^{N ! c(N) N}$.

Lemma 8.2.5. Let $G$ be a connected semisimple group over $\mathbb{Q}_{p}$ and let $K \subset G\left(\mathbb{Q}_{p}\right)$ be a maximal special compact subgroup. Assume that $p>\iota\left(\left|\mu_{G}\right|\right)$. Then, for every $a \in G\left(\mathbb{Q}_{p}\right)$, either $a \in K$ or $\operatorname{deg}_{K}(a) \geq p$.

8.2.6. Proof of Theorem 8.2.1. We may assume $K$ is of the form (8.1.4). Then for every $a \in G\left(\mathbb{A}_{f}\right)$ we have $\operatorname{deg}_{K}(a)=\prod_{p} \operatorname{deg}_{K_{p}}(a)$. Assume $\operatorname{deg}_{K}(a) \leq d$. If $p \notin \mathcal{P}$ and $p>\max \left\{d, \iota\left(\left|\mu_{G}\right|\right)\right\}$, Lemma 8.2.5 shows that $a \in K_{p}$. But then the conclusion follows from Lemma 8.2.2 applied to the finitely many $p$ which are in $\mathcal{P}$ or $\leq \max \left\{d, \iota\left(\left|\mu_{G}\right|\right)\right\}$.

Remark 8.2.7. Assume that $K$ is of the form (8.1.4) and let $n_{d}\left(G\left(\mathbb{A}_{f}\right), K\right)$ denote the number of double classes $K a K$ for $a \in G\left(\mathbb{A}_{f}\right)$ with $\operatorname{deg}_{K}(a) \leq d$. Then

$$
n_{d}\left(G\left(\mathbb{A}_{f}\right), K\right) \leq \prod_{p \in \mathcal{P} \text { or } p \leq d} n_{d}\left(G\left(\mathbb{Q}_{p}\right), K_{p}\right)
$$

8.3. Degree of local Hecke operators. This section is devoted to the proof of Lemmas 8.2.2 and 8.2.5. For an anisotropic group $G$ over $\mathbb{Q}_{p}$, the group $G\left(\mathbb{Q}_{p}\right)$ is compact [Prasad 1982]. So it is enough to prove Lemmas 8.2.2 and 8.2.5 for isotropic groups $G$. Then we can use (avatars of) the Bruhat-Tits decomposition attached to the euclidean building of $G\left(\mathbb{Q}_{p}\right)$, which expresses explicitly the degree of local Hecke operators in terms of the extended affine Weyl group of $G\left(\mathbb{Q}_{p}\right)$. For Lemma 8.2.2, we may assume that $G$ is simply connected (Lemma 8.1.3). Under this assumption, the parametrizing group of the Bruhat-Tits decomposition is a Coxeter group (the affine Weyl group) and computations are easy. For Lemma 8.2.5, we can no longer resort to Lemma 8.1.3 and thus have to handle the Bruhat-Tits decomposition for possibly non-simply connected $G$. There, the parametrizing group of the Bruhat-Tits decomposition (the extended affine Weyl group) is a semidirect product of a Coxeter group by a finite group of nonpreserving type automorphisms, which make computations slightly more technical. As this is 
possibly less known to nonexperts, we include an expository section, which we try to keep as self-contained as possible.

8.3.1. Review of Bruhat-Tits theory. Let $G$ be a connected semisimple isotropic group over $\mathbb{Q}_{p}$ with $\mathbb{Q}_{p}$-split maximal torus $S$. The principle of Bruhat-Tits theory is to attach to $G\left(\mathbb{Q}_{p}\right)$ a discrete euclidean building endowed with a strongly transitive action of $G\left(\mathbb{Q}_{p}\right)$. The existence of this building is essentially equivalent to the datum of a generalized Tits system $\left(G\left(\mathbb{Q}_{p}\right), B, N\right)$, and once the existence of $\left(G\left(\mathbb{Q}_{p}\right), B, N\right)$ is established, the axioms of Tits systems gives a combinatorial description of the compact subgroups of $G\left(\mathbb{Q}_{p}\right)$ containing $B$ in terms of the extended affine Weyl group $N / N \cap B$.

We assume the reader is familiar with the formalism of Tits systems and buildings [Bourbaki 1968, Chapitre IV; Bruhat and Tits 1972, §1, §2; Brown 1989; Garrett 1997]. We review below the construction of the euclidean Bruhat-Tits building attached to $G\left(\mathbb{Q}_{p}\right)$ and summarize in 8.3.1.6 the consequences of this construction which we will need. We follow closely the survey [Rousseau 2009], which provides a synthetic introduction to the classical [Tits 1979; Bruhat and Tits 1972; 1984a; 1984b].

Given a group $H$ acting on a set $X$ and an element $x \in X$, we write $H_{x}$ for the stabilizer of $x$ in $H$.

Let $X^{*}(S)$ and $X_{*}(S)$ denote the groups of characters and cocharacters of $S$, respectively. These are free $\mathbb{Z}$-modules of rank $r=\operatorname{dim}(S)$, dual to each other via the evaluation pairing $X^{*}(S) \times X_{*}(S) \rightarrow \mathbb{Z}$. Set $V(S):=X_{*}(S) \otimes_{\mathbb{Z}} \mathbb{R}$ and $N:=\operatorname{Nor}_{G}(S)\left(\mathbb{Q}_{p}\right), Z:=\operatorname{Cen}_{G}(S)\left(\mathbb{Q}_{p}\right)$. The action of $N$ on $S$ by conjugation yields

$$
v^{v}: N \rightarrow N / Z \hookrightarrow \operatorname{Aut}_{\mathrm{GrAlg} / \mathbb{Q}_{p}}(S) \hookrightarrow \operatorname{Aut}_{\mathbb{Z}}\left(X_{*}(S)\right) \hookrightarrow \operatorname{GL}(V(S)) .
$$

8.3.1.1. The vectorial part $W^{v}$ of the extended affine Weyl group. The torus $S$ acts on the Lie algebra $\mathfrak{g}$ of $G$, which decomposes accordingly as $\mathfrak{g}=\bigoplus_{\alpha \in X^{*}(S)} g_{\alpha}$, where $\mathfrak{g}_{\alpha} \subset \mathfrak{g}$ denotes the eigenspace corresponding to the character $\alpha \in X^{*}(S)$. Let $\Phi$ denote the set of all $0 \neq \alpha \in X^{*}(S)$ such that $\mathfrak{g}_{\alpha} \neq 0$. Then $\Phi \subset V(S)^{*}$ is a (not necessarily reduced) root system in the usual sense. Let $W^{v}$ denote the Weyl group of $\Phi$; we endow $V(S)$ with a $W^{v}$-invariant scalar product. For every $\alpha \in \Phi$, let $r_{\alpha} \in W^{v}$ denote the orthogonal reflexion fixing $\operatorname{ker}(\alpha)$. The morphism $v^{v}: N \rightarrow \mathrm{GL}(V(S))$ induces an isomorphism $v^{v}: N / Z \stackrel{\sim}{\rightarrow} W^{v}$. We can describe more precisely elements corresponding to the reflexions $r_{\alpha}, \alpha \in \Phi$. For every $\alpha \in \Phi$ there exists a unique connected unipotent group $U_{\alpha} \hookrightarrow G$ normalized by $\operatorname{Cen}_{G}(S)$ and such that the induced embedding of Lie algebras $\mathfrak{u}_{\alpha} \hookrightarrow \mathfrak{g}$ identifies $\mathfrak{u}_{\alpha}$ with $\mathfrak{g}_{\alpha} \oplus \mathfrak{g}_{2 \alpha}$. Also, for every $1 \neq u \in U_{\alpha}\left(\mathbb{Q}_{p}\right)$, the intersection $U_{-\alpha}\left(\mathbb{Q}_{p}\right) u U_{-\alpha}\left(\mathbb{Q}_{p}\right) \cap N$ consists of a single element $m(u)$, and $m(u)$ has the property that $v^{v}(m(u))=r_{\alpha} \in N / Z \simeq W^{v}$ [Borel and Tits 1965, §5]. 
8.3.1.2. The extended affine Weyl group $\widehat{W}$. As the restriction map

$$
X^{*}\left(\operatorname{Cen}_{G}(S)\right) \rightarrow X^{*}(S)
$$

has finite cokernel of order, say, $m \geq 1$, for every $z \in Z$ there exists a unique $v(z) \in V(S)$ such that $\chi(v(z))=-\frac{1}{m} v_{p}(m \chi(z)), \chi \in X^{*}(S)$. This defines a morphism $v: Z \rightarrow V(S)$ characterized by the fact that $\chi(v(s))=-v_{p}(\chi(s))$ for $\chi \in X^{*}(S), s \in S\left(\mathbb{Q}_{p}\right)$. Set $\widehat{T}:=v(Z) \subset V(S)$ and $Z_{0}:=\operatorname{ker}(v) \subset Z$, which is a compact open subgroup containing $S\left(\mathbb{Z}_{p}\right) \simeq\left(\mathbb{Z}_{p}^{\times}\right)^{r}$. The morphism $v: Z \rightarrow V(S)$ extends [Rousseau 2009, Proposition 11.3] to a morphism $v: N \rightarrow \mathrm{GA}(V(S))$ with values in the group $\mathrm{GA}(V(S))$ of affine transformations of $V(S)$ and which makes the following diagram commute:

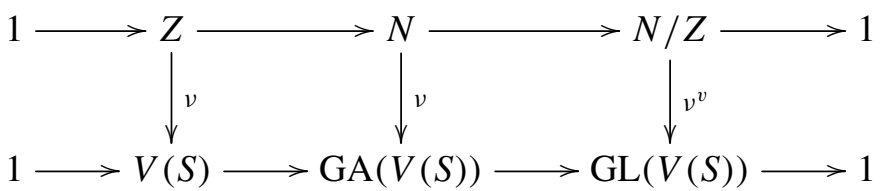

where the left and right vertical arrows are the morphisms defined above. The extension $v: N \rightarrow \mathrm{GA}(V(S))$ is unique up to $\mathrm{GA}(V(S))$-conjugacy. Write $\widehat{W}:=v(N)$.

8.3.1.3. The Weyl group $W$ and the standard apartment $A(S)$. For every $\alpha \in \Phi$ and $1 \neq u \in U_{\alpha}\left(\mathbb{Q}_{p}\right), v(m(u)) \in \widehat{W}$ is an orthogonal reflexion with hyperplane $H(u)$ of direction $\operatorname{ker}(\alpha)$, and

$$
W:=\left\langle v(m(u)) \mid 1 \neq u \in U_{\alpha}\left(\mathbb{Q}_{p}\right), \alpha \in \Phi\right\rangle \triangleleft \widehat{W}
$$

is a discrete affine reflexion group. Let $A(S):=(V(S), W)$ denote the corresponding apartment (see [Bruhat and Tits 1972, §1.3; Rousseau 2009, Part I]) and $\mathcal{F}(S)$ the set of its facets. For a special point $x \in A(S)$, we have $W \simeq T \rtimes W_{x}$ with $T \subset \widehat{T}$ and $W_{x} \simeq \widehat{W}_{x} \simeq W^{v}$. Fix from now on a special point $0 \in A(S)$.

8.3.1.4. Parahoric and parabolic subgroups. For every $\alpha \in \Phi$ and $1 \neq u \in U_{\alpha}\left(\mathbb{Q}_{p}\right)$, there exists a unique $\phi_{\alpha}(u) \in \mathbb{R}$ such that $H(u)=\alpha^{-1}\left(-\phi_{\alpha}(u)\right)$. Set $\phi_{\alpha}(1)=+\infty$. This defines a map $\left.\left.\phi_{\alpha}: U_{\alpha}\left(\mathbb{Q}_{p}\right) \rightarrow\right]-\infty,+\infty\right]$ with the property that $U_{\alpha, \lambda}:=$ $\phi_{\alpha}^{-1}([\lambda,+\infty]) \subset U_{\alpha}\left(\mathbb{Q}_{p}\right)$ is a subgroup for every $\lambda \in \mathbb{R}$. For every $x \in A(S)$, let $P_{x} \subset G\left(\mathbb{Q}_{p}\right)$ denote the subgroup generated by $Z_{0}$ and the $U_{\alpha,-\alpha(x)}, \alpha \in \Phi$. When $F=C$ is a chamber, $P_{0}(C)$ is called an Iwahori subgroup. For every facet $F \subset A(S)$, define the parahoric subgroup of type $F$ to be the subgroup $P_{0}(F) \subset G\left(\mathbb{Q}_{p}\right)$ generated by $Z_{0}$ and the $U_{\alpha, \lambda}$ for $\alpha \in \Phi, \lambda \in \mathbb{R}$ such that $F \subset \phi_{\alpha}^{-1}([-\lambda,+\infty])$. Also let $N_{F} \subset N$ denote the pointwise stabilizer of $F$ in $N$. Then the group $P(F):=N_{F} P_{0}(F) \subset G\left(\mathbb{Q}_{p}\right)$ is called the parabolic subgroup of type $F$.

8.3.1.5. The building $\mathcal{I}^{a}\left(G, \mathbb{Q}_{p}\right)$. Let $\mathcal{I}^{a}\left(G, \mathbb{Q}_{p}\right)$ be the quotient of $G\left(\mathbb{Q}_{p}\right) \times A(S)$ by the equivalence relation $(g, x) \sim\left(g^{\prime}, x^{\prime}\right)$ if and only if there exists $n \in N$ such 
that $x^{\prime}=v(n) x$ and $g^{-1} g^{\prime} n \in P_{x} N_{x}$. The action of $G\left(\mathbb{Q}_{p}\right)$ by left multiplication on the first factor of $G\left(\mathbb{Q}_{p}\right) \times A(S)$ induces an action on $\mathcal{I}^{a}\left(G, \mathbb{Q}_{p}\right)$. One shows that $\mathcal{I}^{a}\left(G, \mathbb{Q}_{p}\right)$ is a euclidean building — the Bruhat-Tits building of $G$ over $\mathbb{Q}_{p}$ with set of apartments $g A(S), g \in G\left(\mathbb{Q}_{p}\right)$ and set of facets $g \mathcal{F}(S), g \in G\left(\mathbb{Q}_{p}\right)$. The pointwise stabilizer of a facet $g F$ is $g P(F) g^{-1}$, the stabilizer of $g A(S)$ is $g N g^{-1}$ and the pointwise stabilizer of $g A(S)$ is $g Z_{0} g^{-1}$.

For the following, we refer to [Tits 1979, §3.4, 3.5]. Let $F$ be a facet in $\mathcal{I}^{a}\left(G, \mathbb{Q}_{p}\right)$ and let $G\left(\mathbb{Q}_{p}\right)_{F} \subset G\left(\mathbb{Q}_{p}\right)$ denote the stabilizer of $F$ in $G\left(\mathbb{Q}_{p}\right)$. Then there exists a unique smooth affine group scheme $\mathcal{G}_{F}$ over $\mathbb{Z}_{p}$ with generic fiber $G$ and with the property that $\mathcal{G}_{F}\left(\mathcal{O}_{k}\right)=G(k)_{F}$ for every finite unramified extension $k / \mathbb{Q}_{p}$ (here we implicitly identify $\mathcal{I}^{a}\left(G, \mathbb{Q}_{p}\right)$ with its image in $\left.\mathcal{I}^{a}(G, k)\right)$. Write $\mathcal{G}_{F, \mathbb{F}_{p}}^{\text {red }}:=\mathcal{G}_{F, \mathbb{F}_{p}}^{\circ} / R_{u}\left(\mathcal{G}_{F, \mathbb{F}_{p}}\right)$ for the connected reductive part of the reduction modulo $p$ of $\mathcal{G}_{F}$; as $G$ is residually quasisplit, $\mathcal{G}_{F, \mathbb{F}_{p}}^{\text {red }}$ is quasisplit. The link of $F$ in $\mathcal{I}^{a}\left(G, \mathbb{Q}_{p}\right)$ is the spherical building of $\mathcal{G}_{F, \mathbb{F}_{p}}^{\text {red }}$. In particular, when $F$ is of codimension $1, \mathcal{G}_{F, \mathbb{F}_{p}}^{\text {red }}$ has semisimple $\mathbb{F}_{p}$-rank 1 and its spherical building is 0-dimensional with vertices corresponding to its minimal parabolic subgroups. More precisely, let $R$ denote the canonical set of generators of $W$ (the reflexions with respect to the walls of a chamber). If $F$ is of type $R \backslash\{r\}$, let $d_{r}$ denote the dimension of $\mathcal{G}_{F, \mathbb{F}_{p}}^{\text {red }} / P$, where $P$ is a minimal parabolic subgroup. Then the number of vertices in the link of $F$ is $p^{d_{r}}+1$. The classification shows that $d_{r}=1,2,3$ but we only need the fact that $d_{r} \geq 1$. This number can also be interpreted as the number of chambers in $\mathcal{I}^{a}\left(G, \mathbb{Q}_{p}\right)$ containing $F$; that is, $\operatorname{deg}_{B}(r)+1$. $^{3}$

8.3.1.6. Non-type-preserving automorphisms. The action of $G\left(\mathbb{Q}_{p}\right)$ on $\mathcal{I}^{a}\left(G, \mathbb{Q}_{p}\right)$ is strongly transitive ${ }^{3}$ but not type-preserving in general. More precisely, let $G_{\circ} \subset G\left(\mathbb{Q}_{p}\right)$ denote the subgroup acting on $\mathcal{I}^{a}\left(G, \mathbb{Q}_{p}\right)$ by type-preserving automorphisms; this is the subgroup generated by $N_{\circ}:=v^{-1}(W)$ and the $U_{\alpha}\left(\mathbb{Q}_{p}\right), \alpha \in \Phi$. The action of $G_{\circ}$ on $\mathcal{I}^{a}\left(G, \mathbb{Q}_{p}\right)$ remains strongly transitive [Garrett 1997, §17.7]. Set $B:=P_{0}(C)$ for a chamber $C$ in $A(S)$; note that $B \subset G_{\circ}$. Then $\left(G_{\circ}, B, N_{\circ}\right)$ is the Tits system induced by the strongly transitive, type-preserving action of $G_{\circ}$ on $\mathcal{I}^{a}\left(G, \mathbb{Q}_{p}\right)$ [Garrett 1997, §5.2]. In particular we get the standard Bruhat-Tits decompositions [Garrett 1997, §5.1-5.4]. The formalism of Tits systems (or $B N$ pairs) extends (formally) to the "generalized" Tits system $\left(G\left(\mathbb{Q}_{p}\right), B, N\right)$. This is explained in [Bourbaki 1968, Chapitre IV, §2, Exercice 8; Garrett 1997, §5.5, $\S 14.7$ ] (see also [Borel 1976, §3.1; Tits 1979, §2.5]), which we briefly summarize.

${ }^{3}$ This follows from the fact that the action of $G\left(\mathbb{Q}_{p}\right)$ on $\mathcal{I}^{a}\left(G, \mathbb{Q}_{p}\right)$ is strongly transitive (that is, transitive on the set of embeddings of a chamber into an apartment): if $C$ and $r C$ are two chambers in a given apartment $A$ with wall $F$ and if $C^{\prime}$ is another chamber in $\mathcal{I}^{a}\left(G, \mathbb{Q}_{p}\right)$ containing $F$ then there exists an apartment $A^{\prime}$ containing $C, C^{\prime}$ and $g \in G\left(\mathbb{Q}_{p}\right)$ such that $g A=A^{\prime}, g C=C$. So $g \in B$. Also, the chambers $C=g C$ and $g r C$ have the same wall $g F=F$ in $A^{\prime}$, which forces $g r C=C^{\prime}$. This shows that the chambers containing $F$ in $\mathcal{I}^{a}\left(G, \mathbb{Q}_{p}\right)$ are $C$ and the chambers $b r C, b \in B$. 
(1) (structure of $\widehat{W}$ ) Set $Z_{\circ}:=Z \cap G_{\circ}$. Since $N_{\circ}=v^{-1}(W)$, we have $W \simeq N_{\circ} / B \cap N_{\circ}$ and the following commutative diagram of short exact sequences:

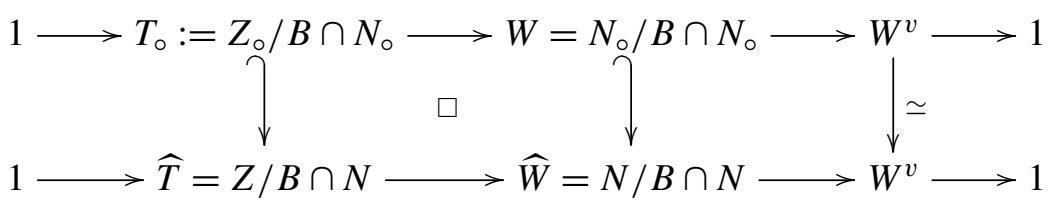

Let $\Psi:=Z / Z_{\circ}$ denote the cokernel of the right (and middle) vertical arrows. As the extension $1 \rightarrow T_{\circ} \rightarrow \widehat{T} \rightarrow \Psi \rightarrow 1$ splits, $\widehat{W}=W \rtimes \Psi$ and an explicit complement of $W$ in $\widehat{W}$ is

$$
\Psi=\frac{P(C) \cap N}{B \cap N} \simeq \frac{N_{C}(B \cap N)}{B \cap N} .
$$

The order of $\Psi$ is bounded from above by a constant which only depends on the Coxeter diagram $\Delta(W, R)$ of $(W, R)$. Indeed, $\Psi$ injects into $\operatorname{Aut}(\Delta(W, S))$ and stabilizes the connected components of $\Delta(W, R)$. In our case, we can also show that $\Psi$ is abelian. This follows for instance from the explicit description given in [Tits $1979, \S 2.5]$. Namely, if $S^{\text {sc }} \subset G^{\text {sc }}$ is a $\mathbb{Q}_{p}$-split maximal torus mapping into $S$, $Z^{\text {sc }}:=\operatorname{Cen}_{G^{\mathrm{sc}}}\left(S^{\mathrm{sc}}\right)\left(\mathbb{Q}_{p}\right)$ and $Z^{\prime}:=\phi\left(\mathbb{Q}_{p}\right)\left(Z^{\mathrm{sc}}\right)$, then $\Psi \simeq Z / Z^{\prime} Z_{0}$. In particular, a rough upper bound for $|\Psi|$ is $|\Psi| \leq \iota\left(\left|\mu_{G}\right|\right)$.

(2) (double cosets) The map $\hat{w} \rightarrow B \hat{w} B$ induces a bijection

$$
\widehat{W} \stackrel{\sim}{\rightarrow} B \backslash G\left(\mathbb{Q}_{p}\right) / B
$$

(3) (subgroups of $G\left(\mathbb{Q}_{p}\right)$ containing $\left.B\right)$ Recall that $R$ denotes the canonical set of generators of $W$, and let $\mathcal{R}$ denote the set of pairs $\left(\Psi^{\prime}, R^{\prime}\right)$ with $\Psi^{\prime} \subset \Psi$ a subgroup and $R^{\prime} \subset R$ a subset normalized by $\Psi^{\prime}$. For a subset $R^{\prime} \subset R$, let $W_{R^{\prime}} \subset W$ denote the subgroup generated by $R^{\prime}$; if $R^{\prime} \subsetneq R$ the group $W_{R^{\prime}}$ is finite. Then the map

$$
\left(\Psi^{\prime}, R^{\prime}\right) \rightarrow P_{\left(\Psi^{\prime}, R^{\prime}\right)}:=B W_{R^{\prime}} \Psi^{\prime} B:=\bigsqcup_{\hat{w} \in W_{R^{\prime}} \Psi^{\prime}} B \hat{w} B
$$

induces a bijection from $\mathcal{R}$ to the set of subgroups of $G\left(\mathbb{Q}_{p}\right)$ containing $B$. Furthermore, a subgroup $P_{\left(\Psi^{\prime}, R^{\prime}\right)} \subsetneq G\left(\mathbb{Q}_{p}\right)$ is a maximal compact subgroup if and only if $R^{\prime} \subsetneq R, \Psi^{\prime}=\operatorname{Nor}_{\Psi}\left(R^{\prime}\right)$ and $\Psi^{\prime}$ acts transitively on $R \backslash R^{\prime}$.

8.3.2. Proof of Lemma 8.2.2. The exact sequence

$$
1 \rightarrow \mu_{G}\left(\mathbb{Q}_{p}\right) \rightarrow G^{\mathrm{sc}}\left(\mathbb{Q}_{p}\right) \rightarrow G\left(\mathbb{Q}_{p}\right) \rightarrow \mathrm{H}^{1}\left(\mathbb{Q}_{p}, \mu_{G}\left(\overline{\mathbb{Q}_{p}}\right)\right)
$$

shows that $p^{\mathrm{sc}}\left(\mathbb{Q}_{p}\right): G^{\mathrm{sc}}\left(\mathbb{Q}_{p}\right) \rightarrow G\left(\mathbb{Q}_{p}\right)$ has finite kernel and finite cokernel. So, from Lemma 8.1.3, we may assume that $G$ is simply connected and that $K=B \subset G\left(\mathbb{Q}_{p}\right)$ is an Iwahori subgroup. Then, from 8.3.1.6(3), we have the 
Bruhat-Tits decomposition

$$
G\left(\mathbb{Q}_{p}\right)=\bigsqcup_{w \in W} B w B
$$

As $(W, R)$ is a Coxeter system, every $w \in W$ can be written as $w=r_{1} \cdots r_{\ell(w)}$ with $r_{1}, \ldots, r_{\ell(w)} \in R$ and $\ell(w) \in \mathbb{Z}_{\geq 0}$ minimal (called the length of $w$ ); the elements $r_{1}, \ldots, r_{\ell}(w)$ are then unique up to permutation. Furthermore, we have

$$
\operatorname{deg}_{B}(w)=\operatorname{deg}_{B}\left(r_{1}\right) \cdots \operatorname{deg}_{B}\left(r_{\ell(w)}\right)
$$

with $\operatorname{deg}_{B}(r)=p^{d_{r}}$ and $d_{r}=1,2,3$ for every $r \in R$ (see 8.3.1.5). In particular, $\operatorname{deg}_{B}(w) \geq p^{\ell(w)}$. The conclusion then follows from the fact that there are only finitely many elements of bounded length in a Coxeter group.

8.3.3. Proof of Lemma 8.2.5. From 8.3.1.6(3), $K \subset G\left(\mathbb{Q}_{p}\right)$ is of the form

$$
K=\bigsqcup_{\hat{w} \in W_{R^{\prime}} \Psi^{\prime}} B \hat{w} B
$$

for a subset $R^{\prime} \subsetneq R$ and a subgroup $\Psi^{\prime} \subset \Psi$ such that $\Psi^{\prime}=\operatorname{Nor}_{\Psi}\left(R^{\prime}\right)$ and $\Psi^{\prime}$ acts transitively on $R \backslash R^{\prime}$. Write $\widehat{W}_{K}:=W_{R^{\prime}} \Psi^{\prime}$. Then, for every $\hat{w}=w \psi \in \widehat{W}$ we can compute, using $B w B w^{\prime} B=B w w^{\prime} B$,

$$
\begin{aligned}
K \hat{w} K & =\Psi^{\prime} B W_{R^{\prime}} B w \psi B W_{R^{\prime}} B \Psi^{\prime}=\Psi^{\prime} B W_{R^{\prime}} B w B \psi W_{R^{\prime}} \psi^{-1} B \psi \Psi^{\prime} \\
& =\Psi^{\prime} B W_{R^{\prime}} w \psi W_{R^{\prime}} \psi^{-1} B \psi \Psi^{\prime}=B \widehat{W}_{K} \hat{w} \widehat{W}_{K} B .
\end{aligned}
$$

That is,

$$
K \hat{w} K=\bigsqcup_{\lambda \in \widehat{W}_{K} \hat{w} \widehat{W}_{K}} B \lambda B
$$

As a result,

On the other hand,

$$
|B \backslash K \hat{w} K|=\sum_{\lambda \in \widehat{W}_{K} \hat{w} \widehat{W}_{K}} \operatorname{deg}_{B}(\lambda)
$$

$$
|B \backslash K \hat{w} K|=\operatorname{deg}_{K}(\hat{w})[K: B]=\operatorname{deg}_{K}(\hat{w}) \sum_{\mu \in \widehat{W}_{K}} \operatorname{deg}_{B}(\mu) .
$$

From this, we get

$$
\operatorname{deg}_{K}(\hat{w})=\frac{\sum_{\lambda \in \widehat{W}_{K} \hat{w} \widehat{W}_{K}} \operatorname{deg}_{B}(\lambda)}{\sum_{\mu \in \widehat{W}_{K}} \operatorname{deg}_{B}(\mu)}
$$

Now, we can compute explicitly

$$
\sum_{\mu \in \widehat{W}_{K}} \operatorname{deg}_{B}(\mu)=\sum_{\psi \in \Psi} \sum_{w \in W_{R^{\prime}}} \operatorname{deg}_{B}(w)=|\Psi|\left(1+\sum_{1 \neq w \in W_{R^{\prime}}} \operatorname{deg}_{B}(w)\right) \equiv|\Psi|[p] .
$$


Write

$$
\widehat{W}_{K} \backslash \widehat{W}_{K} \hat{w} \widehat{W}_{K}=\mathcal{S}_{1} \sqcup \mathcal{S}_{2},
$$

where $\mathcal{S}_{1}$ denotes the set of left cosets $\widehat{W}_{K} \lambda$ such that $\widehat{W}_{K} \lambda \cap \Psi \neq \varnothing$. Then, for $C \in \mathcal{S}_{1}$ we have

$$
\sum_{\lambda \in C} \operatorname{deg}_{B}(\lambda)=\sum_{\lambda \in \widehat{W}_{K}} \operatorname{deg}_{B}(\lambda)
$$

and hence

$$
\operatorname{deg}_{K}(\hat{w})=\left|\mathcal{S}_{1}\right|+\frac{\sum_{C \in \mathcal{C}_{2}} \sum_{\lambda \in C} \operatorname{deg}_{B}(\lambda)}{\sum_{\mu \in \widehat{W}_{K}} \operatorname{deg}_{B}(\mu)} .
$$

But for $C \in \mathcal{S}_{2}$ and $\lambda \in C$ we have $\lambda=w \psi$ with $1 \neq w \in W$ and $\psi \in \Psi$. In particular, $\operatorname{deg}_{B}(\lambda)=\operatorname{deg}_{B}(w)$ is a power of $p . A s \operatorname{deg}_{K}(\hat{w})$ is an integer and $\sum_{\mu \in \widehat{W}_{K}} \operatorname{deg}_{B}(\mu) \equiv|\Psi|[p]$ is nonzero (this is where we use the assumption $\left.p>\imath\left(\left|\mu_{G}\right|\right)(\geq|\Psi|)\right)$, it is thus enough to prove that if $\hat{w} \notin \widehat{W}_{K}$ then $\mathcal{S}_{2} \neq \varnothing$. This follows from the maximality of $K$. Indeed, otherwise, we may assume that $\hat{w}=\psi \in \Psi \backslash \Psi^{\prime}$. Then the following holds:

for every $w \in \widehat{W}_{K}$ there exists $\psi(w, \psi) \in \Psi \backslash \Psi^{\prime}, w(w, \psi) \in \widehat{W}_{K}$

$$
\text { such that } \psi w=w(w, \psi) \psi(w, \psi) \text {. }
$$

But then,

$$
\widehat{W}_{K} \psi(w, \psi) \widehat{W}_{K}=\widehat{W}_{K} \psi \widehat{W}_{K}
$$

Thus $\psi(w, \psi)$ satisfies again $(*)$. This shows that the subgroup generated by $\widehat{W}_{K}$ and $\psi$ in $\widehat{W}$ is of the form $\widehat{W}_{K} \rtimes \Psi^{\prime \prime}$ for some subgroup $\Psi^{\prime} \subsetneq \Psi^{\prime \prime} \subset \Psi$, which contradicts the maximality of $K$.

8.4. Proof of 7.2.2. Recall that we may assume that $\Gamma=G(\mathbb{Q}) \cap K$ for a compact open subgroup $K$ as in (8.1.4). So we will take $K=\prod_{p} K_{p}$ with $K_{p} \subset G\left(\mathbb{Q}_{p}\right)$ compact open (which we could even assume to be maximal) for every $p$ and hyperspecial for $p \notin \mathcal{P}$, where $\mathcal{P}$ denotes the finite set of primes where $G$ ramifies. Also, recall that $\Gamma^{-} \subset G\left(\mathbb{A}_{f}\right)$ denotes the closure of $\Gamma$ for the adelic topology.

Lemma 8.4.1. For every $a \in G(\mathbb{Q})$ the canonical map $\varphi_{a}: \Gamma \backslash \Gamma a \Gamma \rightarrow \Gamma^{-} \backslash \Gamma^{-} a \Gamma^{-}$ is bijective. In particular $\operatorname{deg}_{\Gamma}(a)=\operatorname{deg}_{\Gamma^{-}}(a)$.

Proof. We first prove that $\varphi_{a}$ is injective. Let $\Gamma a \gamma, \Gamma a \gamma^{\prime} \in \Gamma \backslash \Gamma a \Gamma$ be such that $\Gamma^{-} a \gamma=\Gamma^{-} a \gamma^{\prime}$; that is, there exists $\gamma^{-} \in \Gamma^{-}$such that $a \gamma=\gamma^{-} a \gamma^{\prime}$. But then $\gamma^{-}=a \gamma \gamma^{\prime-1} a^{-1} \in \Gamma^{-} \cap G(\mathbb{Q})=\Gamma$. Thus $\Gamma a \gamma=\Gamma a \gamma^{\prime}$. We now show that $\varphi_{a}$ is surjective. As $\varphi_{a}: \Gamma \backslash \Gamma a \Gamma \hookrightarrow \Gamma^{-} \backslash \Gamma^{-} a \Gamma^{-}$is injective and both sets are finite, it is enough to prove that $\operatorname{deg}_{\Gamma^{-}}(a) \leq \operatorname{deg}_{\Gamma}(a)$. For this, fix a set of representatives $R$ 
of $\Gamma \backslash \Gamma a \Gamma$ and observe that

$$
\Gamma^{-} a \Gamma^{-}=(\Gamma a \Gamma)^{-}=\left(\bigsqcup_{b \in R} \Gamma b\right)^{-}=\bigcup_{b \in R}(\Gamma b)^{-}=\bigcup_{b \in R} \Gamma^{-} b .
$$

Here, the first equality follows from the fact that $\Gamma^{-}$is compact (to prove that $\left.(\Gamma a \Gamma)^{-} \subset \Gamma^{-} a \Gamma^{-}\right)$.

Lemma 8.4.2. The canonical map $\varphi: \Gamma \backslash G(\mathbb{Q}) / \Gamma \rightarrow K \backslash G\left(\mathbb{A}_{f}\right) / K$ has finite fibers. More precisely, for every $a \in G(\mathbb{Q}),\left|\varphi^{-1}(\varphi(a))\right| \leq \operatorname{deg}_{K}(a)$.

Proof. Let $a \in G(\mathbb{Q})$ and let $R \subset G(\mathbb{Q})$ be a set of representatives for $\varphi^{-1}(K a K)$. Since $\Gamma=G(\mathbb{Q}) \cap K$, the map $\Gamma \backslash G(\mathbb{Q}) \rightarrow K \backslash G\left(\mathbb{A}_{f}\right)$ is injective, hence restricts to an injective map

$$
\Gamma \backslash \bigsqcup_{b \in R} \Gamma b \Gamma=\bigsqcup_{b \in R} \Gamma \backslash \Gamma b \Gamma \rightarrow K \backslash K a K .
$$

Because the union is disjoint, we get

$$
|R| \leq\left|\bigsqcup_{b \in R} \Gamma \backslash \Gamma b \Gamma\right| \leq|K \backslash K a K|=\operatorname{deg}_{K}(a) .
$$

For simply connected groups $G$ of noncompact type, the proof is now complete: let $n_{d}(G(\mathbb{Q}), \Gamma)$ denote the number of double classes $\Gamma a \Gamma$ with $a \in G(\mathbb{Q})$ and $\operatorname{deg}_{\Gamma}(a) \leq d$. By strong approximation, $\Gamma^{-}=K$. So $\operatorname{deg}_{\Gamma}(a)=\operatorname{deg}_{K}(a)$ (Lemma 8.4.1) and

$$
n_{d}(G(\mathbb{Q}), \Gamma) \leq d n_{d}(G(\mathbb{Q}), K) \leq d n_{d}\left(G\left(\mathbb{A}_{f}\right), K\right)
$$

(Lemma 8.4.2). So the conclusion follows from Theorem 8.2.1.

In the non-simply connected case, we can no longer apply Lemma 8.4.1 directly.

Lemma 8.4.3. There exists an integer $r(G, K) \geq 1$ and a compact normal subgroup $H \subset K$ such that $H \equiv \Gamma^{-}$and for every $p, H_{p} \subset G\left(\mathbb{Q}_{p}\right)$ is compact open and $\left[K_{p}: H_{p}\right] \leq r(G, K)$.

Proof. Set $K_{p}^{\mathrm{sc}}=\left(p^{\mathrm{sc}}\right)^{-1}\left(K_{p}\right) \subset G^{\mathrm{sc}}\left(\mathbb{Q}_{p}\right)$ and $K^{\mathrm{sc}}:=\prod_{p} K_{p}^{\mathrm{sc}} \subset G\left(\mathbb{A}_{f}\right)$. Then $K_{p}^{\mathrm{sc}} \subset G^{\mathrm{sc}}\left(\mathbb{Q}_{p}\right)$ is compact open for every $p$ and hyperspecial for $p \notin \mathcal{P}$. Set $H_{p}:=\operatorname{Cor}_{K_{p}}\left(p^{\mathrm{sc}}\left(K_{p}^{\mathrm{sc}}\right)\right)$ for $p \in \mathcal{P}$ and $H_{p}:=p^{\mathrm{sc}}\left(K_{p}^{\mathrm{sc}}\right)$ otherwise. We claim that $H=\prod_{p} H_{p} \subset K$ works. Indeed, for $p \notin \mathcal{P}$ the short exact sequence

$$
1 \longrightarrow \mu_{G} \longrightarrow G^{\mathrm{sc}} \stackrel{p^{\mathrm{sc}}}{\longrightarrow} G \longrightarrow 1
$$

extends to a short exact sequence of smooth group schemes over $\mathbb{Z}_{p}$ with $K_{p}^{\mathrm{sc}}=$ $G^{\mathrm{sc}}\left(\mathbb{Z}_{p}\right), K_{p}=G\left(\mathbb{Z}_{p}\right)$. Taking the reduction modulo $p$ and $\overline{\mathbb{F}}_{p}$-points, we obtain a 
short exact sequence of finite groups

$$
1 \longrightarrow \mu_{G}\left(\overline{\mathbb{F}}_{p}\right) \longrightarrow G^{\mathrm{sc}}\left(\overline{\mathbb{F}}_{p}\right) \stackrel{p^{\mathrm{sc}}}{\longrightarrow} G\left(\overline{\mathbb{F}}_{p}\right) \longrightarrow 1 .
$$

Since $\mathbb{Z}_{p}^{\text {ur }}$ (the integral closure of $\mathbb{Z}_{p}$ in the maximal unramified extension $\mathbb{Q}_{p}^{\text {ur }}$ of $\mathbb{Q}_{p}$ ) is Henselian, by smoothness (see [Platonov and Rapinchuk 1994, Lemma 6.5, p. 295] for details) we get a short exact sequence

$$
1 \longrightarrow \mu_{G}\left(\mathbb{Z}_{p}^{\mathrm{ur}}\right) \longrightarrow G^{\mathrm{sc}}\left(\mathbb{Z}_{p}^{\mathrm{ur}}\right) \stackrel{p^{\mathrm{sc}}}{\longrightarrow} G\left(\mathbb{Z}_{p}^{\mathrm{ur}}\right) \longrightarrow 1 .
$$

Taking the $\operatorname{Gal}\left(\mathbb{Q}_{p}^{\mathrm{ur}} / \mathbb{Q}_{p}\right)$-invariants, we obtain

$$
H_{p}=\operatorname{ker}\left(K_{p} \rightarrow \mathrm{H}^{1}\left(\mathrm{Gal}\left(\mathbb{Q}_{p}^{\mathrm{ur}} / \mathbb{Q}_{p}\right), \mu_{G}\left(\mathbb{Z}_{p}^{\mathrm{ur}}\right)\right)\right) .
$$

In particular, $H_{p}$ is normal in $K_{p}$ (thus $H$ is normal in $K$ as required) and

$$
\left[K_{p}: H_{p}\right] \leq\left|\mathrm{H}^{1}\left(\operatorname{Gal}\left(\mathbb{Q}_{p}^{\mathrm{ur}} / \mathbb{Q}_{p}\right), \mu_{G}\left(\mathbb{Z}_{p}^{\mathrm{ur}}\right)\right)\right| \leq\left|\mu_{G}\right| .
$$

The last inequality comes from the fact that $\operatorname{Gal}\left(\mathbb{Q}_{p}^{\text {ur }} / \mathbb{Q}_{p}\right) \simeq \hat{\mathbb{Z}}$ is procyclic, so that by [Serre 1968, Chapitre XIII, §1, Proposition 1],

$$
\mathrm{H}^{1}\left(\operatorname{Gal}\left(\mathbb{Q}_{p}^{\mathrm{ur}} / \mathbb{Q}_{p}\right), \mu_{G}\left(\mathbb{Z}_{p}^{\mathrm{ur}}\right)\right)=\mu_{G}\left(\mathbb{Z}_{p}^{\mathrm{ur}}\right)_{\mathrm{Gal}\left(\mathbb{Q}_{p}^{\mathrm{ur}} / \mathbb{Q}_{p}\right)}
$$

(the maximal trivial $\operatorname{Gal}\left(\mathbb{Q}_{p}^{\mathrm{ur}} / \mathbb{Q}_{p}\right)$-quotient of $\mu_{G}\left(\mathbb{Z}_{p}^{\mathrm{ur}}\right)$ ). So we can take

$$
r(G, K):=\max \left\{\left[K_{p}: H_{p}\right] \mid p \in \mathcal{P} \text { or } p<p_{K}\right\} \cup\left\{\left|\mu_{G}\right|\right\} .
$$

It remains to show that $\Gamma^{-}, H \subset G\left(\mathbb{A}_{f}\right)$ are commensurable. As $\Gamma^{\mathrm{sc}} \subset G^{\mathrm{sc}}(\mathbb{Q})$ is arithmetic and $p^{\mathrm{sc}}: G^{\mathrm{sc}} \rightarrow G$ is surjective, the group $p^{\mathrm{sc}}\left(\Gamma^{\mathrm{sc}}\right) \subset G(\mathbb{Q})$ is again arithmetic [Platonov and Rapinchuk 1994, Theorem 4.1], and hence $\left[\Gamma: p^{\mathrm{sc}}\left(\Gamma^{\mathrm{sc}}\right)\right]$ is finite. Thus $\left[\Gamma^{-}: p^{\mathrm{sc}}\left(\left(\Gamma^{\mathrm{sc}}\right)^{-}\right)\right]\left(\leq\left[\Gamma: p^{\mathrm{sc}}\left(\Gamma^{\mathrm{sc}}\right)\right]\right)$ is finite as well. But, by strong approximation, $\left(\Gamma^{\mathrm{sc}}\right)^{-}=K^{\mathrm{sc}}$ and by the continuity of $p^{\mathrm{sc}}$ and the compactness of $\left(\Gamma^{\mathrm{sc}}\right)^{-}$,

$$
p^{\mathrm{sc}}\left(\Gamma^{\mathrm{sc}}\right)^{-}=p^{\mathrm{sc}}\left(\left(\Gamma^{\mathrm{sc}}\right)^{-}\right)=p^{\mathrm{sc}}\left(K^{\mathrm{sc}}\right)=H .
$$

Lemma 8.4.4. For every integer $d \geq 1$ there are only finitely many double classes $K a K \in K \backslash G(\mathbb{Q}) / K$ with $\operatorname{deg}_{\Gamma^{-}}(a) \leq d$.

Proof. Let $a \in G(\mathbb{Q})$ with $\operatorname{deg}_{\Gamma^{-}}(a) \leq d$ and let $H$ be as in Lemma 8.4.3. Then $d C_{H, \Gamma^{-}} \geq \operatorname{deg}_{H}(a)=\prod_{p} \operatorname{deg}_{H_{p}}(a)$ and $\operatorname{deg}_{H_{p}}(a) \geq \operatorname{deg}_{K_{p}}(a) / r(G, K)$ (Lemma 8.1.1). Set

$$
\mu(G, K, d):=\max \left\{\iota\left(\left|\mu_{G}\right|\right), r(G, K) d C_{H, \Gamma^{-}}\right\} .
$$

Then by Lemma 8.2.5, for $p \notin \mathcal{P}, p>\mu(G, K, d)$, we have $a \in K_{p}$. Set $v(G, K, d):=\iota\left(\left|\mu_{G}\right|\right) d r(G, K) C_{H, \Gamma^{-}}$and

$$
N(G, K, d):=|\mathcal{P}|+|\{p \notin \mathcal{P}: p \leq v(G, K, d)\}| .
$$


Then by Lemma 8.1.1,

$$
\begin{aligned}
\operatorname{deg}_{K}(a) & =\prod_{p \in \mathcal{P}} \operatorname{deg}_{K_{p}}(a) \prod_{p \notin \mathcal{P}, p \leq \nu(G, K, d)} \operatorname{deg}_{K_{p}}(a) \\
& \leq r(G, K)^{N(G, K, d)} \operatorname{deg}_{H}(a) \leq r(G, K)^{N(G, K, d)} d C_{H, \Gamma^{-}} .
\end{aligned}
$$

The conclusion thus follows from Theorem 8.2.1.

8.4.5. End of the proof of Theorem 7.2.2. Let $a \in G(\mathbb{Q})$ such that $\operatorname{deg}_{\Gamma}(a) \leq d$. Then $\operatorname{deg}_{\Gamma^{-}}(a)=\operatorname{deg}_{\Gamma}(a) \leq d$ (Lemma 8.4.1). So there are only finitely many possibilities for the set of double classes $K a K \in K \backslash G(\mathbb{Q}) / K$ (Lemma 8.4.4), and hence only finitely many possibilities for the set of double classes $\Gamma a \Gamma \in \Gamma \backslash G(\mathbb{Q}) / \Gamma$ (Lemma 8.4.2).

Remark 8.4.6. Fix from now on $K_{0} \subset G\left(\mathbb{A}_{f}\right)$ compact open of the form (8.1.4). Let $K \subset G\left(\mathbb{A}_{f}\right)$ be an arbitrary compact open subgroup and set $\Gamma:=K \cap G(\mathbb{Q})$, $\Gamma_{0}:=K_{0} \cap G(\mathbb{Q})$. Then, the proof yields an explicit estimate

$$
n_{d}(G(\mathbb{Q}), \Gamma) \leq A\left[\Gamma_{0}: \Gamma \cap \Gamma_{0}\right]^{2}\left(d C_{\Gamma_{0}, \Gamma}\right) B^{\alpha\left(d C_{\left.\Gamma_{0}, \Gamma\right)}\right) \ln \left(d C_{\Gamma_{0}, \Gamma}\right)+\beta\left(d C_{\Gamma_{0}, \Gamma}\right)+\gamma},
$$

where $A, B, \alpha, \beta, \gamma$ are absolute constants depending only on the group-theoretical data $\Gamma_{0}, \mathcal{P}, W_{G}$, etc., but not on $d$ nor on $\Gamma$.

\section{Alternative approaches to Theorem 7.2.2}

9.1. An ergodic proof of Theorem 7.2.2. The argument below was explained to us by Hee Oh. We use the notation of [Eskin and Oh 2006, Theorem 1.2]. If we have infinitely many distinct $\Gamma a_{i} \Gamma$ with degree bounded by $d$, then the associated $\Delta(G)$-invariant measures $\tilde{v}_{a_{i}}$ have a weak limit $\tilde{v}$. There are two possibilities for $\tilde{v}$ as discussed in the proof: either $\tilde{v}$ is supported in the closed $\Delta(G)$-invariant measure or is a $G \times G$ invariant measure. In the first case, the proof shows that the sequence $\left[\left(e, a_{i}\right)\right] \Delta(G)$ has a constant subsequence; or equivalently, that the sequence $\Gamma a_{i} \Gamma$ has a constant subsequence, contradicting the assumption that they are distinct. The second case where $\tilde{v}$ is $G \times G$-invariant cannot happen, since this is equivalent to $\Gamma \backslash \Gamma a_{i} \Gamma$ being equidistributed in $\Gamma \backslash G(\mathbb{R})$; but $\Gamma \backslash \Gamma a_{i} \Gamma$ has at most $d$ points, so cannot possibly be equidistributed.

We do not know whether Theorem 8.2.1 can be recovered from Theorem 7.2.2, and so be proved by ergodic techniques. In any case, our proof relies on different arguments (Bruhat-Tits, strong approximation) and is effective.

It was also mentioned to us by an anonymous referee that, when $G$ is $\mathbb{Q}$-split, [Gan and Oh 2003, Proposition 6.1] gives an effective bound for the degree of Hecke operators; the proof of [Gan and Oh 2003, Proposition 6.1] involves elements of Bruhat-Tits theory in the split case. 
9.2. Masser-Wüstholz isogeny theorem. One key ingredient of the proof of [Orr 2013, Theorem 1.5(ii)] is (a generalization to finitely generated fields of characteristic 0 of) the Masser-Wüstholz isogeny theorem [Masser and Wüstholz 1993; Orr 2013, Theorem 5.2]. Using it, the existence of closed Galois-generic points on Shimura varieties of abelian type (Theorem A) and technical arguments from Orr's thesis, we can give an alternative (and, again, effective) proof of the fact that on a connected Shimura variety of adjoint abelian type, there are only finitely many Hecke operators of bounded degree.

Lemma 9.2.1. Let $\left(G, X^{+}\right)$be a connected Shimura datum of abelian type. Then for every arithmetic subgroup $\Gamma \subset G(\mathbb{Q}), s \in \operatorname{Sh}_{\Gamma}\left(G, X^{+}\right)$with residue field $k=k(s)$ and integer $d \geq 1$, there are only finitely many $t \in \widehat{T}_{\Gamma}(s)$ with $[k(t): k] \leq d$.

Proof. It is enough to prove the assertion when $G$ is adjoint and for an ordinary Hecke orbit. By [Orr 2013, Theorem 4.6], this case, in turn, reduces to the case of an ordinary Hecke orbit in the Siegel moduli space $\mathcal{A}_{g}:=\operatorname{Sh}_{\mathrm{GSp}_{2 g}}(\mathbb{Z})\left(\mathrm{GSp}_{2 g}, \mathcal{H}_{g}^{+}\right)$. This allows one to use the modular interpretation of $\mathcal{A}_{g}$ as a coarse moduli space for $g$-dimensional principally polarized abelian varieties and the fact that Hecke orbits on $\mathcal{A}_{g}$ correspond to isogeny classes of such objects (here we say that $\left(A, \lambda_{A}\right)$ is isogenous to $\left(B, \lambda_{B}\right)$ if there is an isogeny $f: A \rightarrow B$ and an integer $N \geq 1$ such that $\left.f^{\vee} \circ \lambda_{B} \circ f=N \lambda_{A}\right)$. Let $a \in \mathcal{A}_{g}$ with residue field $k=k(a)$ and $b \in T_{\Gamma}(a)$ with $[k(b): k] \leq d$. Over $\bar{k}, a$ and $b$ correspond to isogenous $g$-dimensional principally polarized abelian varieties $\left(A_{\bar{k}}, \lambda_{A \bar{k}}\right)$ and $\left(B_{\bar{k}}, \lambda_{B \bar{k}}\right)$. Let $\delta$ denote the order of $\operatorname{GSp}_{2 g}\left(\mathbb{F}_{3}\right)$. Then ${ }^{4}\left(A_{\bar{k}}, \lambda_{A \bar{k}}\right)$ admits a model $\left(A, \lambda_{A}\right)$ over a finite field extension $L$ of $k$ with $[L: k] \leq \delta$ and $\left(B_{\bar{k}}, \lambda_{B \bar{k}}\right)$ admits a model $\left(B, \lambda_{B}\right)$ over a finite field extension $L(b)$ of $k(b)$ with $[L(b): k(b)] \leq \delta$. From [Orr 2013, Theorem 5.2] (see also [Masser and Wüstholz 1993]), there exist constants $c(A, L)$ and $\kappa(g)$ (independent of $B$ ) and an isogeny $f: A_{\bar{k}} \rightarrow B_{\bar{k}}$ of degree

$$
\operatorname{deg}(f) \leq c(A, L)[L . L(b): L]^{\kappa(g)} \leq\left(c(A, L) \delta^{2 \kappa(g)}\right) d^{\kappa(g)} .
$$

As $f: A_{\bar{k}} \rightarrow B_{\bar{k}}$ is uniquely determined by its kernel, there are, up to $\bar{k}$-isomorphism, only finitely many possibilities for $B_{\bar{k}}$, and thus for $\left(B_{\bar{k}}, \lambda_{B \bar{k}}\right)$ [Milne 1986, Theorem 18.1].

Now, let $\left(G, X^{+}\right)$be a connected Shimura datum of adjoint abelian type, and let $\Gamma \subset G(\mathbb{Q})$ be a neat congruence subgroup. Fix a closed Galois-generic point $s \in \operatorname{Sh}_{\Gamma}\left(G, X^{+}\right)$with residue field $k(s)=k$. Up to replacing $\Gamma$ by a smaller congruence subgroup, we may assume that $s$ is strictly Galois-generic. Then,

\footnotetext{
${ }^{4}$ Explicitly, let $\mathrm{GSp}_{2 g}(3)$ denote the kernel of the reduction modulo-3 morphism $\mathrm{GSp}_{2 g}(\mathbb{Z}) \rightarrow$ $\mathrm{GSp}_{2 g}\left(\mathbb{F}_{3}\right)$ and write $\mathcal{A}_{g, 3}:=\operatorname{Sh}_{\mathrm{GSp}_{2 g}(\mathbb{Z})(3)}\left(\mathrm{GSp}_{2 g}, \mathcal{H}_{g}^{+}\right)$. Then $L$ (resp. $\left.L(b)\right)$ can be taken to be the residue field of any point in the fiber over $a$ (resp. $b$ ) of $\mathcal{A}_{g, 3} \rightarrow \mathcal{A}_{g}$.
} 
- As $s$ is strictly Galois-generic, for every $a \in G(\mathbb{Q})_{+}$and $t \in T_{\Gamma, a}(s)$ we have $\operatorname{deg}_{\Gamma}(a)=[k(t): k]$.

- As $\Gamma$ is neat, for every $a, b \in G(\mathbb{Q})_{+}$, the following properties are equivalent:

(1) $T_{\Gamma, a}(s) \cap T_{\Gamma, b}(s) \neq \varnothing$;

(2) $T_{\Gamma, a}(s)=T_{\Gamma, b}(s)$;

(3) $\Gamma a \Gamma=\Gamma b \Gamma$.

Combining these observations with Lemma 9.2.1, we see that the number of Hecke operators of bounded degree $\leq d$ on $\operatorname{Sh}_{\Gamma}\left(G, X^{+}\right)$is finite and bounded from above by the number of $t \in T_{\Gamma}(s)$ with $[k(t): k] \leq d$.

\section{Acknowledgements}

This work began while the authors stayed at the Institute for Advanced Study; they are very grateful to the institute for its hospitality. The authors also thank Akio Tamagawa for pointing out a gap in an earlier proof of Theorem A and Ziyang Gao for telling us that Theorem B follows from Orr's result in the case of Shimura data of abelian type. Cadoret thanks the Institute for Advanced Study (Princeton) and the Research Institute for Mathematical Sciences (Kyoto). During her time at the IAS she was supported by the NSF grant No DMS-1155. She was also partly supported by the project ANR-10-JCJC 0107 from the ANR. Kret thanks the Institute for Advanced Study (Princeton), the Mathematical Sciences Research Institute (Berkeley), and the Max Planck Institute for Mathematics (Bonn), where parts of this work were carried out. During his time at the IAS (resp. MSRI) he was supported by the NSF under Grant No. DMS 1128155 (resp. No. 0932078000). Finally, he thanks CMLS at the École Polytechnique for a pleasant stay.

\section{References}

[André 1989] Y. André, G-functions and geometry, Aspects of Mathematics E13, Vieweg, Braunschweig, 1989. MR Zbl

[André 2004] Y. André, Une introduction aux motifs (motifs purs, motifs mixtes, périodes), Panoramas et Synthèses 17, Société Mathématique de France, Paris, 2004. MR Zbl

[Baily and Borel 1966] W. L. Baily, Jr. and A. Borel, "Compactification of arithmetic quotients of bounded symmetric domains", Ann. of Math. (2) 84 (1966), 442-528. MR Zbl

[Borel 1966] A. Borel, "Density and maximality of arithmetic subgroups", J. Reine Angew. Math. 224 (1966), 78-89. MR Zbl

[Borel 1976] A. Borel, "Admissible representations of a semi-simple group over a local field with vectors fixed under an Iwahori subgroup", Invent. Math. 35 (1976), 233-259. MR Zbl

[Borel and Harish-Chandra 1962] A. Borel and Harish-Chandra, "Arithmetic subgroups of algebraic groups”, Ann. of Math. (2) 75 (1962), 485-535. MR Zbl

[Borel and Tits 1965] A. Borel and J. Tits, "Groupes réductifs", Inst. Hautes Études Sci. Publ. Math. 27 (1965), 55-150. MR Zbl 
[Bourbaki 1968] N. Bourbaki, Éléments de mathématique, XXXIV: Groupes et algèbres de Lie, chapitres 4 à 6, Actualités Scientifiques et Industrielles 1337, Hermann, Paris, 1968. MR Zbl

[Brown 1989] K. S. Brown, Buildings, Springer, New York, 1989. MR Zbl

[Bruhat and Tits 1972] F. Bruhat and J. Tits, "Groupes réductifs sur un corps local", Inst. Hautes Études Sci. Publ. Math. 41 (1972), 5-251. MR Zbl

[Bruhat and Tits 1984a] F. Bruhat and J. Tits, "Groupes réductifs sur un corps local, II: Schémas en groupes. Existence d'une donnée radicielle valuée”, Inst. Hautes Études Sci. Publ. Math. 60 (1984), 197-376. MR Zbl

[Bruhat and Tits 1984b] F. Bruhat and J. Tits, "Schémas en groupes et immeubles des groupes classiques sur un corps local”, Bull. Soc. Math. France 112:2 (1984), 259-301. MR Zbl

[Burger and Sarnak 1991] M. Burger and P. Sarnak, "Ramanujan duals, II", Invent. Math. 106:1 (1991), 1-11. MR Zbl

[Cadoret 2015] A. Cadoret, "An open adelic image theorem for abelian schemes", Int. Math. Res. Not. 2015:20 (2015), 10208-10242. MR Zbl

[Cadoret and Moonen 2015] A. Cadoret and B. Moonen, "Integral and adelic aspects of the MumfordTate conjecture", preprint, 2015. arXiv

[Cadoret and Tamagawa 2012] A. Cadoret and A. Tamagawa, "A uniform open image theorem for $\ell$-adic representations, I”, Duke Math. J. 161:13 (2012), 2605-2634. MR Zbl

[Cadoret and Tamagawa 2013] A. Cadoret and A. Tamagawa, "A uniform open image theorem for $\ell$-adic representations, II”, Duke Math. J. 162:12 (2013), 2301-2344. MR Zbl

[Cadoret and Tamagawa 2014] A. Cadoret and A. Tamagawa, "On the geometric image of $\mathbb{F}_{\ell}$-linear representations of étale fundamental groups", preprint, 2014, http://www.math.polytechnique.fr/ perso/cadoret/JordanNorilIndepFinal.pdf.

[Clozel et al. 2001] L. Clozel, H. Oh, and E. Ullmo, "Hecke operators and equidistribution of Hecke points", Invent. Math. 144:2 (2001), 327-351. MR Zbl

[Deligne 1971] P. Deligne, "Travaux de Shimura”, exposé no. 389, 123-165 in Séminaire Bourbaki, 1970/1971, Lecture Notes in Mathematics 244, Springer, Berlin, 1971. MR Zb1

[Deligne 1979] P. Deligne, "Variétés de Shimura: interprétation modulaire, et techniques de construction de modèles canoniques", pp. 247-289 in Automorphic forms, representations and L-functions, Part 2 (Corvallis, OR, 1977), edited by A. Borel and W. Casselman, Proceedings of Symposia in Pure Mathematics 33, American Mathematical Society, Providence, RI, 1979. MR Zbl

[Eskin and Oh 2006] A. Eskin and H. Oh, "Ergodic theoretic proof of equidistribution of Hecke points", Ergodic Theory Dynam. Systems 26:1 (2006), 163-167. MR Zbl

[Faltings and Wüstholz 1984] G. Faltings and G. Wüstholz (editors), Rational points (Max-PlanckInstitut für Mathematik, Bonn, 1983/1984), Aspects of Mathematics E6, Vieweg, Braunschweig, 1984. MR Zbl

[Gan and Oh 2003] W. T. Gan and H. Oh, "Equidistribution of integer points on a family of homogeneous varieties: a problem of Linnik", Compositio Math. 136:3 (2003), 323-352. MR $\mathrm{Zbl}$

[Garrett 1997] P. Garrett, Buildings and classical groups, Chapman \& Hall, London, 1997. MR Zbl

[Jantzen 1997] J. C. Jantzen, "Low-dimensional representations of reductive groups are semisimple", pp. 255-266 in Algebraic groups and Lie groups, Austral. Math. Soc. Lect. Ser. 9, Cambridge University Press, 1997. MR Zbl

[Masser and Wüstholz 1993] D. Masser and G. Wüstholz, "Isogeny estimates for abelian varieties, and finiteness theorems", Ann. of Math. (2) 137:3 (1993), 459-472. MR Zbl 
[Milne 1986] J. S. Milne, "Abelian varieties”, pp. 103-150 in Arithmetic geometry (Storrs, CT, 1984), Springer, New York, 1986. MR Zbl

[Milne 2013] J. S. Milne, "Shimura varieties and moduli", pp. 467-548 in Handbook of moduli, Vol. II, edited by G. Farkas and I. Morrison, Adv. Lect. Math. 25, International Press, Somerville, MA, 2013. MR Zbl

[Nori 1987] M. V. Nori, “On subgroups of $\mathrm{GL}_{n}\left(\mathbf{F}_{p}\right)$ ", Invent. Math. 88:2 (1987), 257-275. MR Zbl

[Orr 2013] M. Orr, La conjecture d'André-Pink: Orbites de Hecke et sous-variétés faiblement spéciales, Ph.D. thesis, Université Paris-Sud, 2013, https://tel.archives-ouvertes.fr/tel-00879010.

[Pink 2005] R. Pink, "A combination of the conjectures of Mordell-Lang and André-Oort", pp. 251-282 in Geometric methods in algebra and number theory, edited by F. Bogomolov and Y. Tschinkel, Progr. Math. 235, Birkhäuser, Boston, 2005. MR Zbl

[Platonov and Rapinchuk 1994] V. Platonov and A. Rapinchuk, Algebraic groups and number theory, Pure and Applied Mathematics 139, Academic Press, Boston, 1994. MR Zbl

[Prasad 1982] G. Prasad, "Elementary proof of a theorem of Bruhat-Tits-Rousseau and of a theorem of Tits", Bull. Soc. Math. France 110:2 (1982), 197-202. MR Zbl

[Rousseau 2009] G. Rousseau, "Euclidean buildings", pp. 77-116 in Géométries à courbure négative ou nulle, groupes discrets et rigidités, edited by L. Bessières et al., Sémin. Congr. 18, Société Mathématique de France, Paris, 2009. MR Zbl

[Serre 1968] J.-P. Serre, Corps locaux, Hermann, Paris, 1968. MR

[Serre 1989] J.-P. Serre, Lectures on the Mordell-Weil theorem, Aspects of Mathematics E15, Vieweg, Braunschweig, 1989. MR Zbl

[Serre 1994] J.-P. Serre, "Propriétés conjecturales des groupes de Galois motiviques et des représentations $l$-adiques", pp. 377-400 in Motives (Seattle, 1991), edited by U. Jannsen et al., Proceedings of Symposia in Pure Mathematics 55, American Mathematical Society, Providence, RI, 1994. MR Zbl

[Serre 2013] J.-P. Serre, "Un critère d'indépendance pour une famille de représentations $\ell$-adiques", Comment. Math. Helv. 88:3 (2013), 541-554. MR Zbl

[Tits 1979] J. Tits, "Reductive groups over local fields", pp. 29-69 in Automorphic forms, representations and L-functions (Corvallis, OR, 1977), vol. 1, edited by A. Borel and W. Casselman, Proceedings of Symposia in Pure Mathematics 33, American Mathematical Society, Providence, RI, 1979. MR Zbl

[Ullmo and Yafaev 2013] E. Ullmo and A. Yafaev, "Mumford-Tate and generalised Shafarevich conjectures”, Ann. Math. Qué. 37:2 (2013), 255-284. MR Zbl

Communicated by Bjorn Poonen

Received 2015-09-13 Revised 2016-06-29 Accepted 2016-08-12

anna.cadoret@polytechnique.edu Centre de Mathématiques Laurent Schwartz, Ecole Polytechnique, 91128 Palaiseau, France

arnokret@gmail.com

Faculty of Science, Korteweg-de Vries Instituut, Postbus 94248, 1090 GE Amsterdam, Netherlands 


\section{Algebra \& Number Theory}

msp.org/ant

\section{EDITORS}

MANAGING EDITOR

Bjorn Poonen

Massachusetts Institute of Technology

Cambridge, USA

\author{
EDITORIAL BOARD CHAIR \\ David Eisenbud \\ University of California \\ Berkeley, USA
}

BOARD OF EDITORS

$\begin{aligned} \text { Dave Benson } & \text { University of Aberdeen, Scotland } & \text { Susan Montgomery } & \text { University of Southern California, USA } \\ \text { Richard E. Borcherds } & \text { University of California, Berkeley, USA } & \text { Shigefumi Mori } & \text { RIMS, Kyoto University, Japan } \\ \text { John H. Coates } & \text { University of Cambridge, UK } & \text { Raman Parimala } & \text { Emory University, USA } \\ \text { J-L. Colliot-Thélène } & \text { CNRS, Université Paris-Sud, France } & \text { Jonathan Pila } & \text { University of Oxford, UK } \\ \text { Brian D. Conrad } & \text { Stanford University, USA } & \text { Anand Pillay } & \text { University of Notre Dame, USA } \\ \text { Hélène Esnault } & \text { Freie Universität Berlin, Germany } & \text { Victor Reiner } & \text { University of Minnesota, USA } \\ \text { Hubert Flenner } & \text { Ruhr-Universität, Germany } & \text { Peter Sarnak } & \text { Princeton University, USA } \\ \text { Sergey Fomin } & \text { University of Michigan, USA } & \text { Joseph H. Silverman } & \text { Brown University, USA } \\ \text { Edward Frenkel } & \text { University of California, Berkeley, USA } & \text { Michael Singer } & \text { North Carolina State University, USA } \\ \text { Andrew Granville } & \text { Université de Montréal, Canada } & \text { Vasudevan Srinivas } & \text { Tata Inst. of Fund. Research, India } \\ \text { Joseph Gubeladze } & \text { San Francisco State University, USA } & \text { J. Toby Stafford } & \text { University of Michigan, USA } \\ \text { Roger Heath-Brown } & \text { Oxford University, UK } & \text { Ravi Vakil } & \text { Stanford University, USA } \\ \text { Craig Huneke } & \text { University of Virginia, USA } & \text { Michel van den Bergh } & \text { Hasselt University, Belgium } \\ \text { Kiran S. Kedlaya } & \text { Univ. of California, San Diego, USA } & \text { Marie-France Vignéras } & \text { Université Paris VII, France } \\ \text { János Kollár } & \text { Princeton University, USA } & \text { Kei-Ichi Watanabe } & \text { Nihon University, Japan } \\ \text { Yuri Manin } & \text { Northwestern University, USA } & \text { Efim Zelmanov } & \text { University of California, San Diego, USA } \\ \text { Philippe Michel } & \text { École Polytechnique Fédérale de Lausanne } & \text { Shou-Wu Zhang } & \text { Princeton University, USA }\end{aligned}$

PRODUCTION

production@msp.org

Silvio Levy, Scientific Editor

See inside back cover or msp.org/ant for submission instructions.

The subscription price for 2016 is US $\$ 290$ /year for the electronic version, and $\$ 485 /$ year (+\$55, if shipping outside the US) for print and electronic. Subscriptions, requests for back issues and changes of subscribers address should be sent to MSP.

Algebra \& Number Theory (ISSN 1944-7833 electronic, 1937-0652 printed) at Mathematical Sciences Publishers, 798 Evans Hall \#3840, c/o University of California, Berkeley, CA 94720-3840 is published continuously online. Periodical rate postage paid at Berkeley, CA 94704, and additional mailing offices.

ANT peer review and production are managed by EditFLow ${ }^{\circledR}$ from MSP.

\section{PUBLISHED BY}

- mathematical sciences publishers

nonprofit scientific publishing

http://msp.org/

() 2016 Mathematical Sciences Publishers 


\section{Algebra \& Number Theory}

Volume $10 \quad$ No. $9 \quad 2016$

Torsion pour les variétés abéliennes de type I et II

MARC HINDRY and NICOLAS RATAZZI

Galois-generic points on Shimura varieties

ANNA CADORET and ARNO KRET

Extremality of loci of hyperelliptic curves with marked Weierstrass points

DAWEI CHEN and NiCOLA TARASCA

$\bar{\Re}_{15}$ is of general type

GREGOR BRUNS

A vanishing theorem for weight-one syzygies

LAWRENCE EIN, ROBERT LAZARSFELD and DAVID YANG

1983

Effective cones of cycles on blowups of projective space

IZZET COSKUN, JOHN LESIEUTRE and JOHN CHRISTIAN OTTEM

Cluster algebras and category $\mathrm{O}$ for representations of Borel subalgebras of quantum affine algebras

DAVID HERNANDEZ and BERNARD LECLERC 